\title{
Time-Varying Panel Data Models with an Additive Factor Structure
}

Fei Liu, Jiti Gao and Yanrong Yang

December 2020

Working Paper 42/20 


\title{
Time-Varying Panel Data Models with an Additive Factor Structure
}

\author{
Fei LiU ${ }^{\dagger}$, Jiti GaO ${ }^{\ddagger}$ AND YANROng YANG \\ Nankai University, China ${ }^{\dagger}$ \\ Monash University, Australia ${ }^{\ddagger}$ \\ The Australian National University, Australia $\sharp^{\sharp}$
}

November 13, 2020

\begin{abstract}
Motivated by many key features of real data from economics and finance, we study a semiparametric panel data model with time-varying regression coefficients associated with an additive factor structure. In our model, factor loadings are unknown functions of observable variables which can capture time-variant and heterogeneous covariate information. A profile marginal integration (PMI) method is proposed to estimate unknown coefficient functions, factors and their loadings jointly in a single step, which can result in estimators with closed forms. Asymptotic distributions for the proposed profile estimators are established. Two empirical applications on US stock returns and OECD health care expenditure are provided. Thorough numerical results demonstrate the finite sample performance of our estimation and its advantage over traditional models in the relevant literature.
\end{abstract}

Keywords: Additive factor model; nonparametric kernel estimation; profile marginal integration.

JEL classification: C14, C23, C33

\section{Introduction}

Panel data analysis is popular in social and scientific areas, such as economics, finance, biology, ecology and so on. Recently, various panel data models with factor structures appear in the econometrics literature (see Pesaran, 2006; Bai, 2009; Su et al., 2016, for example). We consider a nonlinear panel data model with an additive factor structure of the form:

$$
y_{i t}=\mathbf{x}_{i t}^{\top} \boldsymbol{\beta}\left(\tau_{t}\right)+\sum_{j=1}^{J} \lambda_{j}\left(V_{j i t}\right) F_{j t}+\varepsilon_{i t},
$$


for $i=1,2, \ldots, N$ and $t=1,2, \ldots, T$, where $\tau_{t}=\frac{t}{T}, \boldsymbol{\beta}(\tau)$ and $\boldsymbol{\lambda}(\mathbf{v})=\left(\lambda_{1}\left(v_{1}\right), \ldots, \lambda_{J}\left(v_{J}\right)\right)^{\top}$ are unknown deterministic functions of $\tau \in[0,1]$ and $\mathbf{v}=\left(v_{1}, v_{2}, \ldots, v_{J}\right)^{\top}$ respectively, $\mathbf{x}_{i t}$ and $\mathbf{V}_{i t}=$ $\left(V_{1 i t}, \ldots, V_{J i t}\right)^{\top}$ are $p$-dimensional and $J$-dimensional observable variables, $\mathbf{F}_{t}=\left(F_{1 t}, \ldots, F_{J t}\right)^{\top}$ are unobservable factors, and $\varepsilon_{i t}$ is an idiosyncratic error term.

Except the time-varying regression coefficient $\boldsymbol{\beta}\left(\tau_{t}\right)$, as can be seen from model (1.1), the observable covariate variables in loadings are also time variant through the time-varying term $V_{j i t}$. In financial studies to model stock returns, for example, $\mathbf{V}_{i t}$ can be time-variant security characteristics, such as size, value, momentum and own-volatility. To reveal the relationship between these characteristics and stock returns, Connor et al. (2012) consider a semiparametric additive factor structure:

$$
y_{i t}=F_{0 t}+\sum_{j=1}^{J} \lambda_{j}\left(V_{j i}\right) F_{j t}+\varepsilon_{i t} .
$$

To estimate model (1.2), Connor et al. (2012) use a recursive procedure that alternates a nonparametric kernel estimation with the ordinary least squares (OLS). For the same model, Ma et al. (2017) propose a quantile regression version of Connor et al. (2012) in order for better modelling of daily stock returns. As an application of model (1.1) in an empirical analysis of stock returns, our methodology allows the time-variation information of firm characteristic to be used to explain the differences in stock returns. This significantly relaxes the time-constant assumption on loadings required by Ma et al. (2017) and Fan et al. (2016).

Fan et al. (2016) extend Model (1.2) in the sense of factor betas or loadings partially explained by observable covariates:

$$
y_{i t}=\sum_{j=1}^{J}\left(\lambda_{j}\left(V_{j i}\right)+\eta_{j i}\right) F_{j t}+\varepsilon_{i t},
$$

where the unobservable loading components $\left\{\eta_{j i}\right\}$ are mean-zero and independent with $\left\{V_{j i}\right\}$ and $\left\{\varepsilon_{i t}\right\}$. For model (1.3), Fan et al. (2016) develop the projected PCA estimators based on the sieve approximation of unknown loading functions and establish consistency theorems. It is worth noting that the existing studies mentioned above only consider the case where $\mathbf{V}_{i t}=$ $\left(V_{1 i t}, \ldots, V_{J i t}\right)^{\top}$ reduces to $\mathbf{V}_{i}=\left(V_{1 i}, \ldots, V_{J i}\right)^{\top}$ that is independent of $t$. In addition to specifying the loadings as unknown functions of observed variables, Su and Wang (2017) consider the case where the loading functions, $\lambda_{j}(\cdot)$ in model (1.1), are assumed to be time-varying functions of the form: $\lambda_{j}\left(\tau_{t}\right)$.

We propose model (1.1) in this paper to extend models (1.2) and (1.3) in the sense of not only incorporating the time-dependent variables, $\mathbf{V}_{i t}$, into the loading functions, but also introducing the explanatory variables $\mathbf{x}_{i t}$ and time-varying coefficients $\boldsymbol{\beta}\left(\tau_{t}\right)$ into the model. Compared with pure factor models, the involvement of $\mathbf{x}_{i t}$ is capable to accommodate more observable information in addition to loading covariates to explain the time-variation and cross-sectional difference of dependent variables in financial studies and economic research. For example, researchers have identified several economic variables other than firm characteristics that can be used to explain 
the time-variation of stock returns. Santos and Veronesi (2006) show the ratio of labour income to consumption plays a significant role in predicting stock returns. Another well-known example is investor sentiment effect discussed by Baker and Wurgler (2006). Baker and Wurgler (2006) present empirical evidence from US stock market that the investor sentiment has significant effects on the time-variation and cross-sectional differences of stock prices. In addition, Baker and Wurgler (2006) reveal that the predictive power of firm characteristics is related to investor sentiment effect in different time periods. These phenomenons cannot be revealed if pure factor models are only used to model stock returns. In Section 5.1, we conduct an empirical study on US stock market and reveal the significance of investor sentiment effect in predicting the excess stock returns, which suggests the importance of introducing an additive factor structure as an unknown functional form of important and relevant characteristics variables.

Meanwhile, we introduce a nonparametric trending function $\boldsymbol{\beta}(\tau)$ into the panel data model to capture the time-variation in the relationship between the explanatory and the dependent variables. The presence of time-varying features has been proved in many fields of economics studies, such as climate economics, financial economics and energy economics (see Chen et al., 2012b; Silvapulle et al., 2017). As a recent example, Silvapulle et al. (2017) reveal a significant time-varying relationship between stock and oil price indices. To capture this feature, several nonparametric and semiparametric models have been proposed in both time-series and panel data literature (see Cai, 2007; Robinson, 2012; Li et al., 2011). In Section 5, we conduct two empirical studies on modelling stock returns and estimating income elasticity of health care expenditure. Time-variations in the relationship between dependent and explanatory variables are revealed in both applications. It confirms the theoretical necessity and practical relevance of using the nonparametric coefficients $\boldsymbol{\beta}(\tau)$.

Before we discuss about the estimation method we are going to propose in Section 2 below, we have some review about two of the relevant estimation methods for nonparametric additive models. The conventional nonparametric marginal integration (MI) method is widely employed by the relevant literature to estimate nonparametric additive models (see Tjøstheim and Auestad, 1994; Newey, 1994). Although the MI method might be inferior to back-fitting techniques in terms of asymptotic efficiency for purely additive models (see Nielsen and Linton, 1998; Mammen et al., 1999), the MI method is not only able to offer closed-form expressions for nonparametric estimators, but also better suited to the framework of semiparametric MI estimation for stationary spatial data discussed in (see Gao et al., 2006). This is due to the fact that the MI method possesses a closed form expression as a function of the linear component in the profile estimation procedure. Taking into account the advantages of the MI method, we develop a profile marginal integration (PMI) method to jointly estimate $\boldsymbol{\beta}(\tau), \boldsymbol{\lambda}(\mathbf{v})$ and $\mathbf{F}_{t}$ involved in model (1.1) in a number of simple steps outlined in Section 2. In comparison with the MI method employed in Gao et al. (2006), for example, the proposed PMI method considerably explores and generalizes the MI ideas to accommodate the nonlinear panel data setting in model (1.1) where the observed dataset $\left\{\left(y_{i t}, \mathbf{x}_{i t}\right)\right\}$ can be nonstationary time series in the time series dimension and 
cross-sectionally dependent in the cross-sectional dimension, the coefficient functions $\left\{\beta\left(\tau_{t}\right)\right\}$ and the loading functions $\left\{\lambda_{j}\left(V_{j i t}\right)\right\}$ are all unknown nonparametrically, and there are unknown latent factors $\left\{F_{j t}\right\}$ involved (see Section 3.2 for the added technical discussion about the generality and novelty of model (1.1) and its estimation procedure as well as the resulting theory in relation to the relevant literature).

In short, our contributions can be summarised as follows.

1. To capture the time-variation and cross-sectional differences in stock returns, we incorporate nonparametric additive factor structure into panel data models and allow the regression coefficients to be time-varying. With this nonparametric model setting, we can make sufficient use of available information in both observable firm characteristics and other predictor variables without introducing the risk of model misspecification for both regression and loading components.

2. The proposed PMI method is developed to jointly estimate the unknown functions in a number of steps outlined in Section 2. The estimation procedure is easy to implement and no numerical approximation is required.

3. For the proposed estimators, we are able to establish asymptotic consistency and distributional results for the proposed estimators, including these for the loadings and factors, under some mild conditions. Moreover, we obtain an optimal rate of convergence for each of the proposed estimators.

4. Nonlinearity and time-variation from both the regression coefficients and factor loadings makes the model more flexible. This flexibility is appropriate in modelling stock returns and OECD health care expenditure data, as is illustrated by its better out-of-sample performance discussed in Section 5.

The rest of this paper is organised as follows: Section 2 describes the estimation method. Section 3 introduces the assumptions and establishes the asymptotic distributions. Section 4 outlines an instruction about how to apply the PMI method in practice. Section 5 applies the proposed estimation method to two empirical examples in financial and health economics, respectively. Simulation experiments are conducted in Section 6. Section 7 briefly concludes this paper. The proofs of the main results are provided in Appendix A. In an online supplementary document, some technical lemmas and their proofs are given in Appendix B, and additional technicalities are available in Appendix C.

We define the following notation: for a matrix $\mathbf{A}$, we denote $\mathbf{A}^{\top}$ as its transpose and $\|\mathbf{A}\|$ as its Frobenius norm: trace $\left(\mathbf{A} \mathbf{A}^{\top}\right)^{1 / 2} ; \lambda_{\min }(\mathbf{A})$ and $\lambda_{\max }(\mathbf{A})$ are the smallest and largest eigenvalues of $\mathbf{A}$, respectively; $\mathbf{I}_{a}$ and $\mathbf{0}_{a}$ are $a \times a$ identity and null matrices, with $\mathbf{0}_{a \times b}$ being $a \times b$ matrix of zeros; " $P$ " denotes convergence in probability and " $D$ " denotes convergence in distribution; we denote $E(\mathbf{a})$ and $E\left(\mathbf{a} \mid \mathbf{b}=\mathbf{b}_{0}\right)$ as the unconditional expectation of $\mathbf{a}$ and the conditional expectation on $\mathbf{b}=\mathbf{b}_{0}$, respectively. 


\section{$2 \quad$ Model and estimation}

In this section, we introduce our basic ideas to estimate the unknown quantities $\boldsymbol{\beta}(\tau), \boldsymbol{\lambda}(\mathbf{v})$ and $\mathbf{F}=\left(\mathbf{F}_{1}, \ldots, \mathbf{F}_{T}\right)^{\top}$. The identification issue is well recognised in the literature on factor models. In this paper, we assume that the unobservable factors are deterministic in the same way as in Connor et al. (2012). These conditions are given in the following assumption.

Assumption 1 (Identification).

(1) For the stochastic loading functions, suppose that:

$$
E\left[\lambda_{j}\left(V_{j i t}\right)\right]=0, \quad E\left[\lambda_{j}^{2}\left(V_{j i t}\right)\right]=1 .
$$

(2) For the deterministic factors, suppose that:

$$
\bar{F}_{j}=\frac{1}{T} \sum_{t=1}^{T} F_{j t}>0 .
$$

In Assumption 1.(1), the loading functions are assumed to have zero mean to ensure the identifiability among the factor components. The conditions on $E\left[\lambda_{j}^{2}\left(V_{j i t}\right)\right]=1$ and $\bar{F}_{j}>0$ are required to uniquely identify and then estimate the loadings and factors.

Let $\gamma_{j t}\left(v_{j}\right)=\lambda_{j}\left(v_{j}\right) F_{j t}$ and $\gamma_{t}(\mathbf{v})=\sum_{j=1}^{J} \gamma_{j t}\left(v_{j}\right)$. We can rewrite model (1.1) as follows:

$$
y_{i t}=\mathbf{x}_{i t}^{\top} \boldsymbol{\beta}\left(\tau_{t}\right)+\sum_{j=1}^{J} \gamma_{j t}\left(V_{j i t}\right)+\varepsilon_{i t} .
$$

Then, we propose using the proposed PMI method to first estimate $\gamma_{t}(\mathbf{v})$ in Step 1 and $\gamma_{j t}\left(v_{j}\right)$ in Step 2 before we finally estimate $\boldsymbol{\beta}(\tau), \lambda_{j}\left(v_{j}\right)$ and $F_{j t}$ in Steps 3 and 4 in the estimation procedure outlined below.

We now introduce the following conditional means to accommodate potential nonstationarity inherited in the data. Let $m_{Y, i}(\tau)=E\left[Y_{i t} \mid \tau_{t}=\tau\right]$ and $\mathbf{m}_{X, i}(\tau)=E\left[\mathbf{x}_{i t} \mid \tau_{t}=\tau\right]$ capture the unknown trends in $Y_{i t}$ and $\mathbf{x}_{i t}$ for individual $i$, respectively. Then it is clear to see that, under Assumption 1, $m_{Y, i}(\tau)=\mathbf{m}_{X, i}^{\top}(\tau) \boldsymbol{\beta}(\tau)$. In view of this, $\boldsymbol{\beta}\left(\tau_{t}\right)$ captures the dynamic long-run relationship between $\mathbf{x}_{i t}$ and $Y_{i t}$.

We summarize the estimation procedure in the following four steps.

(1) Step 1: By regarding $\boldsymbol{\beta}_{t}=\boldsymbol{\beta}\left(\tau_{t}\right)$ as known, $\gamma_{t}(\mathbf{v})$ can be estimated by the nonparametric local linear estimation method. In detail, we have for each given $t$ :

$$
\begin{aligned}
& \gamma_{t}\left(\mathbf{v}, \boldsymbol{\beta}_{t}\right)=E\left[y_{i t}-\boldsymbol{\beta}^{\top}\left(\tau_{t}\right) \mathbf{x}_{i t} \mid \mathbf{V}_{i t}=\mathbf{v}\right] \\
& =E\left[y_{i t}-m_{Y, i}\left(\tau_{t}\right)-\boldsymbol{\beta}^{\top}\left(\tau_{t}\right)\left(\mathbf{x}_{i t}-\mathbf{m}_{X, i}\left(\tau_{t}\right)\right) \mid \mathbf{V}_{i t}=\mathbf{v}\right] .
\end{aligned}
$$

Let $\widetilde{y}_{i t}=y_{i t}-m_{Y, i}\left(\tau_{t}\right)$ and $\widetilde{\mathbf{x}}_{i t}=\mathbf{x}_{i t}-\mathbf{m}_{X, i}\left(\tau_{t}\right)$. We have

$$
\gamma_{t}\left(\mathbf{v}, \boldsymbol{\beta}_{t}\right)=E\left[\widetilde{y}_{i t} \mid \mathbf{V}_{i t}=\mathbf{v}\right]-\boldsymbol{\beta}^{\top}\left(\tau_{t}\right) E\left[\widetilde{\mathbf{x}}_{i t} \mid \mathbf{V}_{i t}=\mathbf{v}\right]
$$




$$
=: \mathcal{H}_{t}^{(0)}(\mathbf{v})-\sum_{l=1}^{p} \beta_{l}\left(\tau_{t}\right) \mathcal{H}_{t}^{(l)}(\mathbf{v})
$$

where $\mathcal{H}_{t}^{(0)}(\mathbf{v})=E\left[\widetilde{y}_{i t} \mid \mathbf{V}_{i t}=\mathbf{v}\right]$ and $\mathcal{H}_{t}^{(l)}(\mathbf{v})=E\left[\widetilde{x}_{i t}^{(l)} \mid \mathbf{V}_{i t}=\mathbf{v}\right]$ with $\widetilde{x}_{i t}^{(l)}$ being the $l$-th element of $\widetilde{\mathbf{x}}_{i t}$. In practice, $\mathcal{H}_{t}^{(0)}(\mathbf{v})$ and $\mathcal{H}_{t}^{(l)}(\mathbf{v})$ can be estimated by the local linear methods. In detail, let $\widehat{m}_{Y, i}(\tau)$ and $\widehat{\mathbf{m}}_{X, i}(\tau)$ be the local linear estimators of $m_{Y, i}(\tau)$ and $\mathbf{m}_{X, i}(\tau)$, respectively.

We then estimate $\mathcal{H}_{N, t}^{(0)}(\mathbf{v})$ and $\mathcal{H}_{N, t}^{(l)}(\mathbf{v})$ by $\mathcal{H}_{t}^{(0)}(\mathbf{v})$ and $\mathcal{H}_{t}^{(l)}(\mathbf{v})$, given respectively by

$$
\mathcal{H}_{N, t}^{(0)}(\mathbf{v})=\widehat{E}\left[\widehat{\widetilde{y}}_{i t} \mid \mathbf{V}_{i t}=\mathbf{v}\right] \quad \text { and } \mathcal{H}_{N, t}^{(l)}(\mathbf{v})=\widehat{E}\left[\widehat{\widetilde{x}}_{i t}^{(l)} \mid \mathbf{V}_{i t}=\mathbf{v}\right]
$$

where $\widehat{\widetilde{y}}_{i t}=y_{i t}-\widehat{m}_{Y, i}\left(\tau_{t}\right), \widehat{\widetilde{x}}_{i t}^{(l)}=x_{i t}^{(l)}-\widehat{m}_{X, i}^{(l)}\left(\tau_{t}\right)$ and $\widehat{m}_{X, i}^{(l)}(\tau)$ is the $l$-th element of $\widehat{\mathbf{m}}_{X, i}\left(\tau_{t}\right)$. The local linear estimator of $\gamma_{t}(\mathbf{v})$, given $\boldsymbol{\beta}_{t}$, can then be obtained by:

$$
\widehat{\gamma}_{t}(\mathbf{v}) \equiv \widehat{\gamma}_{t}\left(\mathbf{v}, \boldsymbol{\beta}_{t}\right)=\mathcal{H}_{N, t}^{(0)}(\mathbf{v})-\sum_{l=1}^{p} \beta_{l}\left(\tau_{t}\right) \mathcal{H}_{N, t}^{(l)}(\mathbf{v})
$$

(2) Step 2: With $\widehat{\gamma}_{t}(\mathbf{v})$, we can estimate $\gamma_{j t}\left(v_{j}\right)$ by the marginal integration method. Let $\mathbf{V}_{i t}^{(-j)}=\left(V_{1 i t}, \ldots, V_{j-1, i t}, V_{j+1, i t}, \ldots, V_{J i t}\right)^{\top}, \mathbf{V}_{i t}^{(-j)}\left(v_{j}\right)=\left(V_{1 i t}, \ldots, V_{j-1, i t}, v_{j}, V_{j+1, i t}, \ldots, V_{J i t}\right)^{\top}$ and $W^{(-j)}(\cdot): \mathrm{R}^{J-1} \rightarrow \mathrm{R}$ be known weight functions with $E\left[W^{(-j)}\left(\mathbf{V}_{i t}^{(-j)}\right)\right]=1$. Let $w_{j}(\cdot)$ be an indicator function satisfying $w_{j}\left(v_{j}\right)=1$ if $v_{j} \in\left[\underline{v}_{j}, \bar{v}_{j}\right]$ and $w_{j}\left(v_{j}\right)=0$ otherwise.

Then for any given $\boldsymbol{\beta}_{t}$, the MI projection function is as follows: for $v_{j} \in\left[\bar{v}_{j}, \underline{v}_{j}\right]$,

$$
\begin{aligned}
& \mathcal{P}_{j t}\left(v_{j}, \boldsymbol{\beta}_{t}\right):=E\left[\gamma_{t}\left(\mathbf{V}_{i t}^{(-j)}\left(v_{j}\right), \boldsymbol{\beta}_{t}\right) W^{(-j)}\left(\mathbf{V}_{i t}^{(-j)}\right)\right] w_{j}\left(v_{j}\right) \\
& =\gamma_{j t}\left(v_{j}, \boldsymbol{\beta}_{t}\right) w_{j}\left(v_{j}\right)+\sum_{k \neq j} E\left[\gamma_{k t}\left(V_{k i t}, \boldsymbol{\beta}_{t}\right) W^{(-j)}\left(\mathbf{V}_{i t}^{(-j)}\right)\right]=\gamma_{j t}\left(v_{j}, \boldsymbol{\beta}_{t}\right)+C_{j},
\end{aligned}
$$

where $C_{j}=\sum_{k \neq j} E\left[\gamma_{k t}\left(V_{k i t}, \boldsymbol{\beta}_{t}\right) W^{(-j)}\left(\mathbf{V}_{i t}^{(-j)}\right)\right]$ is a constant. It implies that

$$
\gamma_{j t}\left(v_{j}, \boldsymbol{\beta}_{t}\right)=\mathcal{P}_{j t}\left(v_{j}, \boldsymbol{\beta}_{t}\right)-C_{j}
$$

For the MI projection function $\mathcal{P}_{j t}\left(v_{j}, \boldsymbol{\beta}_{t}\right)$, we define the following estimator:

$$
\begin{aligned}
& \widehat{\mathcal{P}}_{j t}\left(v_{j}, \boldsymbol{\beta}_{t}\right)=\frac{1}{N} \sum_{i=1}^{N} \widehat{\gamma}_{t}\left(\mathbf{V}_{i t}^{(-j)}\left(v_{j}\right), \boldsymbol{\beta}_{t}\right) W^{(-j)}\left(\mathbf{V}_{i t}^{(-j)}\right) w_{j}\left(v_{j}\right) \\
& =\widehat{\mathcal{P}}_{j t}^{(0)}\left(v_{j}\right)-\sum_{l=1}^{p} \beta_{l}\left(\tau_{t}\right) \widehat{\mathcal{P}}_{j t}^{(l)}\left(v_{j}\right),
\end{aligned}
$$

where $\widehat{\mathcal{P}}_{j t}^{(l)}\left(v_{j}\right)$ is defined as:

$$
\widehat{\mathcal{P}}_{j t}^{(l)}\left(v_{j}\right)=\frac{1}{N} \sum_{i=1}^{N} \mathcal{H}_{N, t}^{(l)}\left(\mathbf{V}_{i t}^{(-j)}\left(v_{j}\right)\right) W^{(-j)}\left(\mathbf{V}_{i t}^{(-j)}\right) w_{j}\left(v_{j}\right),
$$

for $l=0,1, \ldots, p$, and it is an estimated version of

$$
\mathcal{P}_{j t}^{(l)}\left(v_{j}\right)=E\left[\mathcal{H}_{t}^{(l)}\left(\mathbf{V}_{i t}^{(-j)}\left(v_{j}\right)\right) W^{(-j)}\left(\mathbf{V}_{i t}^{(-j)}\right)\right] w_{j}\left(v_{j}\right) .
$$


(3) Step 3: Let $y_{i t}^{*}=\widetilde{y}_{i t}-\sum_{j=1}^{J} \mathcal{P}_{j t}^{(0)}\left(V_{j i t}\right), x_{i t}^{(l) *}=\widetilde{x}_{i t}^{(l)}-\sum_{j=1}^{J} \mathcal{P}_{j t}^{(l)}\left(V_{j i t}\right)$ and $\mathbf{x}_{i t}^{*}=\left(x_{i t}^{(1) *}, \ldots, x_{i t}^{(p) *}\right)^{\top}$. By the local linear approximation, for $\tau \in(0,1)$ :

$$
y_{i t}^{*}-\mathbf{x}_{i t}^{* \top} \boldsymbol{\beta}\left(\tau_{t}\right) \approx y_{i t}^{*}-\mathbf{x}_{i t}^{* \top} \boldsymbol{\beta}(\tau)-\mathbf{x}_{i t}^{* \top} \boldsymbol{\beta}^{\prime}(\tau)\left(\tau_{t}-\tau\right) .
$$

We then estimate $\boldsymbol{\beta}(\tau)$ and $\boldsymbol{\beta}^{\prime}(\tau)$ by minimising the following objective function:

$$
\min _{\mathbf{a}, \mathbf{b}} \sum_{i=1}^{N} \sum_{t=1}^{T}\left(\widehat{y}_{i t}^{*}-\widehat{\mathbf{x}}_{i t}^{* \top}\left(\mathbf{a}+\mathbf{b}\left(\frac{\tau_{t}-\tau}{h_{0}}\right)\right)\right)^{2} K\left(\frac{\tau_{t}-\tau}{h_{0}}\right),
$$

where $\widehat{y}_{i t}^{*}=\widehat{\widetilde{y}}_{i t}-\sum_{j=1}^{J} \widehat{\mathcal{P}}_{j t}^{(0)}\left(V_{j i t}\right), \widehat{x}_{i t}^{(l) *}=\widehat{\widetilde{x}}_{i t}^{(l)}-\sum_{j=1}^{J} \widehat{\mathcal{P}}_{j t}^{(l)}\left(V_{j i t}\right), \widehat{\mathbf{x}}_{i t}^{*}=\left(\widehat{x}_{i t}^{(1) *}, \ldots, \widehat{x}_{i t}^{(p) *}\right)^{\top}$, and $K(\cdot)$ is the kernel function and $h_{0}$ is the bandwidth.

Let $\mathbf{W}(\tau)=\operatorname{diag}\left(K\left(\frac{\tau_{1}-\tau}{h_{0}}\right), \ldots, K\left(\frac{\tau_{T}-\tau}{h_{0}}\right)\right) \otimes I_{N}, \mathcal{Y}_{t}=\left(\widehat{y}_{1 t}^{*}, \ldots, \widehat{y}_{N t}^{*}\right)^{\top}$ and $\mathcal{X}_{t}=\left(\widehat{\mathbf{x}}_{1 t}^{*}, \ldots, \widehat{\mathbf{x}}_{N t}^{*}\right)^{\top}$. Define

$$
\mathcal{X}(\tau)=\left(\begin{array}{c}
\mathcal{X}_{1}, \ldots, \mathcal{X}_{T} \\
\mathcal{X}_{1}\left(\frac{\tau_{1}-\tau}{h_{0}}\right), \ldots, \mathcal{X}_{T}\left(\frac{\tau_{T}-\tau}{h_{0}}\right)
\end{array}\right)^{\top}
$$

The local linear estimator of $\boldsymbol{\beta}(\tau)$ is given by

$$
\widehat{\boldsymbol{\beta}}(\tau)=\left[\mathbf{I}_{p}, \mathbf{0}_{p}\right]\left(\mathcal{X}^{\top}(\tau) \mathbf{W}(\tau) \mathcal{X}(\tau)\right)^{-1} \mathcal{X}^{\top}(\tau) \mathbf{W}(\tau) \mathcal{Y}
$$

where $\mathcal{Y}=\left(\mathcal{Y}_{1}^{\top}, \ldots, \mathcal{Y}_{T}^{\top}\right)^{\top}$

(4) Step 4: By plugging (2.8) into (2.5), we can obtain an estimator for $\gamma_{j t}\left(v_{j}\right)$ up to a constant. Then, a consistent estimator for $\gamma_{j t}\left(v_{j}\right)$ is given by:

$$
\widehat{\gamma}_{j t}\left(v_{j}\right)=\widehat{\mathcal{P}}_{j t}\left(v_{j}, \widehat{\boldsymbol{\beta}}\left(\tau_{t}\right)\right)-\frac{1}{N} \sum_{i=1}^{N} \widehat{\mathcal{P}}_{j t}\left(V_{j i t}, \widehat{\boldsymbol{\beta}}\left(\tau_{t}\right)\right) .
$$

With $\widehat{\gamma}_{j t}\left(v_{j}\right)$, we can estimate $\lambda_{j}\left(v_{j}\right)$ and $F_{j t}$ by identification conditions:

$$
\widehat{\lambda}_{j}\left(v_{j}\right)=\frac{\frac{1}{T} \sum_{t=1}^{T} \widehat{\gamma}_{j t}\left(v_{j}\right)}{\sqrt{\int \frac{1}{T}\left(\sum_{t=1}^{T} \widehat{\gamma}_{j t}\left(v_{j}\right)\right)^{2} d P_{V, j}\left(v_{j}\right)}}
$$

where $P_{V, j}\left(v_{j}\right)$ is the probability distribution of $V_{j i t}$, and

$$
\widehat{F}_{j t}=\left(\sum_{i=1}^{N} \widehat{\lambda}_{j}^{2}\left(V_{j i t}\right)\right)^{-1} \sum_{i=1}^{N} \widehat{\lambda}_{j}\left(V_{j i t}\right) \widehat{\gamma}_{j t}\left(V_{j i t}\right) .
$$

It is pointed out that the unknown parameters $\boldsymbol{\beta}(\tau)$ and $\gamma_{j t}\left(v_{j}\right)$ are estimated jointly by the nonparametric profile method. Then, $\lambda\left(v_{j}\right)$ and $F_{j t}$ can then be recovered from $\widehat{\gamma}_{j t}\left(v_{j}\right)$ under the identification conditions. Throughout the estimation procedure, no iterations are involved.

In the following section, we establish asymptotic distributions for the proposed estimators $\widehat{\boldsymbol{\beta}}(\tau), \widehat{\gamma}_{j t}\left(v_{j}\right), \widehat{\lambda}\left(v_{j}\right)$ and $\widehat{F}_{j t}$. 


\section{$3 \quad$ Asymptotic distributions}

\subsection{Assumptions and the main results}

We now establish asymptotic properties for the proposed PMI estimators. These asymptotic results are structured as follows: Theorem 3.1 establishes an asymptotic distribution for $\widehat{\boldsymbol{\beta}}(\tau)$. Properties of the loading estimators are discussed in Theorem 3.2. The corresponding results of $\widehat{\gamma}_{j t}\left(v_{j}\right)$ and the factor estimators are provided in Theorems 3.3 and 3.4, respectively.

Recall that $\widetilde{\mathbf{x}}_{i t}=\mathbf{x}_{i t}-\mathbf{m}_{X, i}\left(\tau_{t}\right)$. Let $\widetilde{\mathbf{x}}_{t}=\left(\widetilde{\mathbf{x}}_{1 t}, \widetilde{\mathbf{x}}_{2 t} \ldots, \widetilde{\mathbf{x}}_{N t}\right)^{\top}, \mathbf{V}_{t}=\left(\mathbf{V}_{1 t}, \mathbf{V}_{2 t} \ldots, \mathbf{V}_{N t}\right)^{\top}$ and $\boldsymbol{\varepsilon}_{\cdot t}=\left(\varepsilon_{1 t}, \varepsilon_{2 t}, \ldots, \varepsilon_{N t}\right)^{\top}$.

We now introduce regularity conditions. Assumption 2 lists the conditions on weak crosssectional and serial dependence.

Assumption 2 (Weak Dependence).

(1) Suppose that $\left\{\widetilde{\mathbf{x}}_{t}, \mathbf{V}_{t}, \boldsymbol{\varepsilon}_{t}\right\}$ are strictly stationary and $\alpha$-mixing for $t=1,2, \ldots, T$. Let $\alpha_{i n}(|t-s|)$ represent the $\alpha$-mixing coefficient between $\left\{\widetilde{\mathbf{x}}_{i t}, \mathbf{V}_{i t}, \varepsilon_{i t}\right\}$ and $\left\{\widetilde{\mathbf{x}}_{n s}, \mathbf{V}_{n s}, \varepsilon_{n s}\right\}$ satisfying $\sum_{i=1}^{N} \sum_{n=1}^{N} \sum_{t=1}^{T}\left(\alpha_{i n}(t)\right)^{\delta /(4+\delta)}=O(N)$ and $\sum_{i=1}^{N} \sum_{n=1}^{N}\left(\alpha_{i n}(0)\right)^{\delta /(4+\delta)}=O(N)$, where $\delta>0$ is chosen such that $E\left[\left\|\varepsilon_{i t}\right\|^{4+\delta}\right]<\infty$ and $E\left[\left\|\widetilde{\mathbf{x}}_{i t}\right\|^{4+\delta}\right]<\infty$.

(2) Suppose that $\left\{\widetilde{\mathbf{x}}_{i t}, \mathbf{V}_{i t}, \varepsilon_{i t}\right\}$ are identically distributed for all $i=1,2, \ldots, N$.

(3) The errors $\left\{\varepsilon_{i t}\right\}$ are mean-zero and independent of $\left\{\mathbf{F}_{s}, \mathbf{V}_{j s}, \widetilde{\mathbf{x}}_{j s}\right\}$ for all $(i, j, t, s)$.

Assumption 2 is standard and commonly imposed in the panel data literature. Since we remove the trends in $\mathbf{x}_{i t}$ to obtain $\widetilde{\mathbf{x}}_{i t}$, we impose strict stationarity and $\alpha$-mixing conditions on $\widetilde{\mathbf{x}}_{i t}$ in Assumption 2.(1) without losing generality. These conditions are similar to Assumption 1 of Dong et al. (2015) and Assumption A4 of Chen et al. (2012a). Similar conditions are also required by Assumption 3.4 of Fan et al. (2016). Assumption 2.(2) requires the identical distribution of $\left\{\mathbf{V}_{i t}\right\}$ across $i=1,2, \ldots, N$, which is a classical assumption in the relevant literature (see Assumption A1 by Connor et al., 2012, for example). Assumption 2.(3) is reasonable to impose the mutual independence between $\left\{\varepsilon_{i t}\right\}$ and $\left\{\mathbf{F}_{s}, \mathbf{V}_{j s}, \widetilde{\mathbf{x}}_{j s}\right\}$ since we assume that the model errors $u_{i t} \equiv \sum_{j=1}^{J} \lambda_{j}\left(V_{j i t}\right) F_{j t}+\varepsilon_{i t}$ can be further specified in such a way.

Local linear estimation is employed to estimate the functions $\left\{\boldsymbol{\beta}_{i}(\tau), \lambda_{j}\left(v_{j}\right)\right\}$. It is conventional to introduce some mild conditions on the smoothness of these functions as well as the convergence of bandwidth (see Fan and Gijbels, 1996; Li and Racine, 2007). Recall that the bandwidth $h_{0}$ is used in the local linear estimation of $\boldsymbol{\beta}(\tau)$. Let $b$ be the bandwidth used in the kernel estimation $\widehat{\mathbf{m}}_{X, i}(\tau)$ and $\widehat{m}_{Y, i}(\tau)$. Let $h_{j}$ be the bandwidth used in the estimation of $\gamma_{j t}\left(v_{j}\right)$ for $j=1,2, \ldots, J$. Let $p_{V, j}\left(v_{j}\right)$ be the marginal probability density function of $V_{j i t}$. Let $p_{V}(\mathbf{v})$ and $p_{V}^{(-j)}\left(\mathbf{v}^{(-j)}\right)$ be the joint probability density functions of $\mathbf{V}_{i t}$ and $\mathbf{V}_{i t}^{(-j)}$, respectively. Let $H_{J}=\prod_{j=1}^{J} h_{j}$ and $H_{J}^{(-j)}=\prod_{k \neq j} h_{k}$. Define the compact sets: $\Pi_{V}=\left\{\left(v_{1}, \ldots, v_{J}\right) \mid v_{j} \in\left[\bar{v}_{j}, \underline{v}_{j}\right]\right.$, for $\left.j=1, \ldots, J\right\}$ and $\Pi_{V}^{(-j)}=\left\{\left(v_{1}, \ldots, v_{j-1}, v_{j+1}, \ldots, v_{J}\right) \mid v_{k} \in\right.$ $\left[\bar{v}_{k}, \underline{v}_{k}\right]$, for $\left.k=1,2, \ldots, j-1, j+1, \ldots, J\right\}$. 
Assumption 3 (Kernel Estimation).

(1) The unknown deterministic functions $\boldsymbol{\beta}(\tau)$ and $\lambda_{j}\left(v_{j}\right)$ have continuous derivatives of up to the second order on $\tau \in[0,1]$ and $v_{j} \in\left[\bar{v}_{j}, \underline{v}_{j}\right]$, respectively.

(2) The joint probability density functions $\left\{p_{V, j}\left(v_{j}\right), p_{V}(\mathbf{v}), p_{V}^{(-j)}\left(\mathbf{v}^{(-j)}\right)\right\}$ have continuous derivatives of up to the second order in $v_{j} \in\left[\bar{v}_{j}, \underline{v}_{j}\right], \Pi_{V}$ and $\Pi_{V}^{(-j)}$, respectively.

(3) The kernel function $K(\cdot)$ is Lipschitz continuous with compact support on $[-1,1]$.

(4) The bandwidth $h \in\left\{b, h_{0}, h_{1}, \ldots, h_{J}\right\}$ satisfies that $h \rightarrow 0, \lim _{\sup _{N, T \rightarrow \infty}} N T h^{5}<\infty$ and $\lim _{N, T \rightarrow \infty} N T h_{0}=\infty$. In addition, we assume $\lim _{N, T \rightarrow \infty} N h_{0}^{4}<\infty$ and $b / h_{j} \rightarrow 0$ for $j=0,1, \ldots, J$.

The conditions required by Assumption 3 are conventional in the literature on local linear estimation (see Fan and Gijbels, 1996; Chen et al., 2012b, for example). The convergence of bandwidth is assumed in Assumption 3.(4), and the bandwidths selected by many classical methods can satisfy this condition. For example, $h_{j}=c_{0}(N T)^{-1 / 5}$ for $j=1,2, \ldots, 5$ and some constant $c_{0}>0$. For empirical data, some data-driven methods could be used to select optimal bandwidths. Some details about the bandwidth selection are provided in Section 4.2 below. For the kernel function $K(v)$, we define the following integrals: $\mu_{m}=\int v^{m} K(v) d v$ for $m=0,1,2$, and $v_{0}=\int K^{2}(v) d v$.

Before we derive asymptotic covariance matrices, we first introduce some notation. Recall that $x_{i t}^{(l) *}=\widetilde{x}_{i t}^{(l)}-\sum_{j=1}^{J} \mathcal{P}_{j t}^{(l)}\left(V_{j i t}\right)$. Let $\breve{x}_{i t}^{(l)}=\widetilde{x}_{i t}^{(l)}-\mathcal{H}_{t}^{(l)}\left(\mathbf{V}_{i t}\right)$ and $\breve{x}_{i t}^{(l) c}=\mathcal{H}_{t}^{(l)}\left(\mathbf{V}_{i t}\right)-\sum_{j=1}^{J} \mathcal{P}_{j t}^{(l)}\left(V_{j i t}\right)$. It is clear to see that $x_{i t}^{(l) *}=\breve{x}_{i t}^{(l)}+\breve{x}_{i t}^{(l) c}$, and $\breve{x}_{i t}^{(l)}$ and $\breve{x}_{i t}^{(l) c}$ are uncorrelated. Let $\xi_{j i t}\left(v_{j}\right)=$ $p_{V}^{-1}\left(\mathbf{V}_{i t}^{(-j)}\left(v_{j}\right)\right) p_{V}^{(-j)}\left(\mathbf{V}_{i t}^{(-j)}\right) W^{(-j)}\left(\mathbf{V}_{i t}^{(-j)}\right) w_{j}\left(v_{j}\right)$ and $\phi_{j i t}=p_{V, j}\left(V_{j i t}\right) \xi_{j i t}\left(V_{j i t}\right)$. Define that $\widetilde{x}_{i t}^{(l) *}=\breve{x}_{i t}^{(l)}+\breve{x}_{i t}^{(l) c}\left(1-\sum_{j=1}^{J} \phi_{j i t}\right), \widetilde{\mathbf{x}}_{i t}^{*}=\left(\widetilde{x}_{i t}^{(1) *}, \ldots, \widetilde{x}_{i t}^{(p) *}\right)^{\top}$.

In Assumption 4 below, we introduce asymptotic covariance matrices and assume their positive definiteness. In practice, these matrices can be estimated by their sample versions. Detailed derivations are provided in Appendix C.

Assumption 4 (Asymptotic Covariance Matrices).

(1) Let $\boldsymbol{\Sigma}_{A}=\lim _{N \rightarrow \infty} N^{-1} \sum_{i=1}^{N} E\left[\widetilde{\mathbf{x}}_{i 1}^{*} \widetilde{\mathbf{x}}_{i 1}^{* \top}\right]$ and $\sigma_{i}^{2}=E\left[\varepsilon_{i 1}^{2}\right]$. Suppose that $\boldsymbol{\Sigma}_{A}$ is positive definite and $\sigma_{i}^{2}$ is a positive scalar.

(2) Let $\sigma_{i n}^{2}(s)=E\left[\varepsilon_{i 1} \varepsilon_{n, 1+s}\right]$ and $\boldsymbol{\Sigma}_{i n}(s)=E\left[\widetilde{\mathbf{x}}_{i 1}^{*} \widetilde{\mathbf{x}}_{n, 1+s}^{* \top}\right]$. Define

$$
\Sigma_{\beta}^{0}=\lim _{N \rightarrow \infty} N^{-1} v_{0} \sum_{i=1}^{N} \sum_{n=1}^{N}\left(\sum_{s=-\infty}^{\infty} \Sigma_{i n}(s) \sigma_{i n}^{2}(s)\right) .
$$

Suppose that $\boldsymbol{\Sigma}_{\beta}^{0}$ is positive definite. 
(3) Let $\Sigma_{i n}^{V}\left(v_{j}, u_{j}, s\right)=E\left[\xi_{j i 1}\left(v_{j}\right) \xi_{j n, 1+s}\left(v_{j}\right) \mid V_{j i 1}=u_{j}, V_{j n, 1+s}=u_{j}\right]$. Define

$$
\Sigma_{j}^{V}\left(v_{j}\right)=\lim _{N \rightarrow \infty} N^{-1} v_{0} \sum_{i=1}^{N} \sum_{n=1}^{N}\left(\sum_{s=-\infty}^{\infty} \Sigma_{i n}^{V}\left(v_{j}, v_{j}, s\right) \sigma_{i n}^{2}(s)\right) .
$$

Suppose that $\Sigma_{j}^{V}\left(v_{j}\right)$ is a positive scalar.

With the asymptotic covariance matrices being defined in Assumption 4, we can establish the following central limit theorems (CLTs) for the proposed PMI estimators of $\beta(\tau)$ and $\gamma_{j}\left(v_{j}\right)$ in Theorems 3.1 and 3.2 below.

Theorem 3.1 (CLT for $\widehat{\boldsymbol{\beta}}(\tau))$.

Let Assumptions 1-4 hold. If, in addition, $\sum_{j=1}^{J} h_{j}^{2}=o\left(h_{0}^{2}\right)$, then for $\tau \in(0,1)$ and as $N, T \rightarrow \infty$ simultaneously:

$$
\sqrt{N T h_{0}}\left(\widehat{\boldsymbol{\beta}}(\tau)-\boldsymbol{\beta}(\tau)-\mathbf{a}(\tau) h_{0}^{2}\right) \stackrel{D}{\longrightarrow} \mathcal{N}\left(\mathbf{0}_{p}, \boldsymbol{\Sigma}_{\beta}\right),
$$

where $\mathbf{a}(\tau)=\frac{\mu_{2}}{2} \boldsymbol{\beta}^{\prime \prime}(\tau)\left(1+o_{P}(1)\right), \mu_{2}=\int v^{2} K(v) d v, \boldsymbol{\Sigma}_{\beta}=\boldsymbol{\Sigma}_{A}^{-1} \boldsymbol{\Sigma}_{\beta}^{0} \boldsymbol{\Sigma}_{A}^{-1}, \boldsymbol{\Sigma}_{A}$ and $\boldsymbol{\Sigma}_{\beta}^{0}$ are defined in Assumption 4.

Theorem 3.2 (CLT for $\widehat{\lambda}_{j}\left(v_{j}\right)$ ).

Let Assumptions 1-4 hold. If, in addition, $h_{0}^{2}+\sum_{k \neq j} h_{k}^{2}=o\left(h_{j}^{2}\right)$, then for each $j$ and $v_{j} \in\left(\underline{v}_{j}, \bar{v}_{j}\right)$, and as $N, T \rightarrow \infty$ simultaneously:

$$
\sqrt{N T h_{j}}\left(\widehat{\lambda}_{j}\left(v_{j}\right)-\lambda_{j}\left(v_{j}\right)-c_{j}\left(v_{j}\right) h_{j}^{2}\right) \stackrel{D}{\longrightarrow} \mathcal{N}\left(0, \Sigma_{j}^{\lambda}\left(v_{j}\right)\right)
$$

where $c_{j}\left(v_{j}\right)=\frac{\mu_{2}}{2}\left(\lambda_{j}^{\prime \prime}\left(v_{j}\right)-E\left[\lambda_{j}^{\prime \prime}\left(V_{j i t}\right)\right]\right)\left(1+o_{P}(1)\right) . \quad \Sigma_{j}^{\lambda}\left(v_{j}\right)=\bar{F}_{j}^{-2} \Sigma_{j}^{V}\left(v_{j}\right)$. Here, $\bar{F}_{j}=$ $T^{-1} \sum_{t=1}^{T} F_{j t}$ and $\Sigma_{j}^{V}\left(v_{j}\right)$ is defined in Assumption 4 .

In Theorem 3.1, we establish a root-NTh $h_{0}$ consistency for $\widehat{\boldsymbol{\beta}}(\tau)$, which is an optimal rate of convergence for nonparametric estimation of panel data models. In Theorem 3.2, we establish the asymptotic distributions of the loading estimators with an optimal rate of convergence of a root-NTh$h_{j}$. The leading orders of $h_{0}^{2}$ and $h_{j}^{2}$ involved in the bias terms have simple expressions under the conditions: $h_{0}^{2}+\sum_{k \neq j} h_{k}^{2}=o\left(h_{j}^{2}\right)$ and $h_{0}^{2}+\sum_{k \neq j} h_{k}^{2}=o\left(h_{j}^{2}\right)$, respectively. Otherwise, the general forms of the leading orders will become an order of the form: $O_{P}\left(h_{0}^{2}+\sum_{j=1}^{J} h_{j}^{2}\right)$.

In Theorems 3.1 and 3.2, we have established the asymptotic distributions of the slope and loading estimators associated with the asymptotic covariance matrices. For empirical data, we will provide methods to construct sample estimates for these matrices in Appendix C.

Before we proceed to establish asymptotic distributions for the factor estimators, we first impose high-level CLT assumptions on two cross-sectional averages. These conditions are needed because of the weak cross-sectional dependence between $\left\{\mathbf{V}_{i t}, \varepsilon_{i t}\right\}$ and $\left\{\mathbf{V}_{j t}, \varepsilon_{j t}\right\}$. How to establish asymptotic properties for general cross-sectional averages remains unclear. One potential solution is to develop CLTs under an i.i.d assumption across $i$. For example, Connor et al. (2012) impose a cross-sectionally block-independent condition before they establish asymptotic distributions for the loading and factor estimators. Another approach is to assume marginal 
mixing conditions (see Ma et al., 2017). In these assumptions, the dependence on the $i$ direction is assumed in the same way as that in the $t$ direction. However, neither the block i.i.d nor the mixing condition is practical for real data analysis, considering that cross-sectional dependence is not determined by the index distance $|i-j|$ in most empirical studies.

In this paper, we are not going to employ the existing methods for the establishment of asymptotic properties for cross-sectional averages. Instead, we impose the following high-level conditions. Let $\widetilde{\xi}_{j n t}=\frac{1}{N h_{j}} \sum_{i=1}^{N} K\left(\frac{V_{j i t}-V_{j n t}}{h_{j}}\right) \lambda_{j}\left(V_{j i t}\right) \xi_{j n t}\left(V_{j i t}\right)$.

Assumption 5 (CLTs).

(1) For $j=1,2, \ldots, J$, suppose that:

$$
\frac{1}{\sqrt{N h_{j}}} \sum_{i=1}^{N} K\left(\frac{V_{j i t}-v_{j}}{h_{j}}\right) \xi_{j i t}\left(v_{j}\right) \varepsilon_{i t} \stackrel{D}{\longrightarrow} \mathcal{N}\left(0, \Sigma_{j t}^{V}\left(v_{j}\right)\right),
$$

where $\Sigma_{j t}^{V}\left(v_{j}\right)$ is assumed to be a positive scalar.

(2) For $j=1,2, \ldots, J$, suppose that:

$$
\frac{1}{\sqrt{N}} \sum_{i=1}^{N} \widetilde{\xi}_{j i t} \varepsilon_{i t} \stackrel{D}{\longrightarrow} \mathcal{N}\left(0, \Sigma_{F, t}^{0}\right)
$$

where $\Sigma_{F, t}^{0}$ is assumed to be a positive scalar.

Assumption 5 can be justified with additional assumptions on the cross-sectional dependence structure, and the form of covariance matrices can be formulated. For example, with either the i.i.d or an $\alpha$-mixing condition, we can show that:

$$
\Sigma_{j t}^{V}\left(v_{j}\right)=\sigma_{\varepsilon}^{2} \int \frac{W_{(-j)}^{2}\left(\mathbf{v}^{(-j)}\right) p_{V}^{(-j) 2}\left(\mathbf{v}^{(-j)}\right)}{p_{V}(\mathbf{v})} d \mathbf{v}^{(-j)},
$$

where $\sigma_{\varepsilon}^{2}=E\left[\varepsilon_{11}^{2}\right]$. This result is consistent with the conventional covariance matrix for the MI estimator (Theorem 3.2 of Gao et al., 2006) for the stationary spatial data setting.

When the structure of weak cross-sectional dependence is specified, we can derive $\Sigma_{j t}^{V}\left(v_{j}\right)$. An example is given by Assumption B5 of Lee and Robinson (2016). According to their assumption, we have $\varepsilon_{i t}=\sum_{k=1}^{\infty} \phi_{i k} e_{k t}$, where $\left\{e_{k t}\right\}$ are independent random variables with zero mean and unit variance, and $\phi_{i n}$ are real constants such that $\sum_{i=1}^{N} \sum_{n=1}^{N}\left|\sum_{k=1}^{\infty} \sum_{m=1}^{\infty} \phi_{i k} \phi_{n m}\right|<C N$. With this condition, we can show that Assumption 5.(1) holds with:

$$
\Sigma_{j t}^{V}\left(v_{j}\right)=N^{-1} v_{0} \sum_{i=1}^{N} \sum_{n=1}^{N} \Sigma_{i n}^{V}\left(v_{j}, v_{j}\right) \gamma_{i n}
$$

where $\gamma_{i n}=\sum_{k=1}^{\infty} \sum_{m=1}^{\infty} \phi_{i k} \phi_{n m}$ and $\Sigma_{j t}^{V}\left(v_{j}\right)$ is defined in Assumption 4.

With Assumption 5, we are now ready to establish asymptotic distributions for $\widehat{\gamma}_{j t}\left(v_{j}\right)$ and $\widehat{F}_{j t}$. 
Theorem 3.3 (CLT for $\widehat{\gamma}_{j t}\left(v_{j}\right)$ ).

Let Assumptions 2-3 and 5 hold. As $N, T \rightarrow \infty$ simultaneously, for each $j$ and $t$ and $v_{j} \in\left(\underline{v}_{j}, \bar{v}_{j}\right)$ :

$$
\sqrt{N h_{j}}\left(\widehat{\gamma}_{j t}\left(v_{j}\right)-\gamma_{j t}\left(v_{j}\right)\right) \stackrel{D}{\longrightarrow} \mathcal{N}\left(0, \Sigma_{j t}^{V}\left(v_{j}\right)\right)
$$

where $\Sigma_{j t}^{V}\left(v_{j}\right)$ is defined in Assumption 5.

Theorem 3.4 (CLT for $\widehat{F}_{j t}$ ).

Let Assumptions 1-3 and 5 hold. As $N, T \rightarrow \infty$ simultaneously, for each $j$ and any given $t$ :

$$
\sqrt{N}\left(\widehat{F}_{j t}-F_{j t}\right) \stackrel{D}{\longrightarrow} \mathcal{N}\left(0, \Sigma_{t}^{F}\right)
$$

where $\Sigma_{t}^{F}=E\left[\lambda\left(V_{j i t}\right)^{2}\right]^{-2} \Sigma_{F, t}^{0}$ and $\Sigma_{F, t}^{0}$ is defined in Assumption 5.

In Theorem 3.3, we have established the asymptotic distribution of $\widehat{\gamma}_{j t}\left(v_{j}\right)$ with an optimal rate of convergence of an order of $N h_{j}$, without necessarily requiring the identification conditions in Assumption 1. We have also established the asymptotic distribution of $\widehat{F}_{j t}$ in the above Theorem 3.4.

In oder to show the ease of implementation of the proposed estimation procedure outlined in Section 2, we provide an instruction for computational implementations and applications of the proposed PMI method in practice.

\subsection{Discussion about special cases}

There are some special but important cases of model (1.1) that should be discussed. We focus on the following cases.

Case 1: Where $F_{j t} \equiv 1$, model (1.1) reduces to a nonparametric additive time-varying regression model. In this case, Theorems 3.1-3.3 remain true and unchanged. Theorem 3.4 obviously becomes redundant. Section C.2 of the supplementary document adds some technical details about this case. Section C.3 also discusses about how to deal with the case where the factors are stochastic.

Case 2: When the time-varying coefficient vector $\beta(\tau)$ reduces to $\beta(\tau) \equiv \beta_{0}$, a vector of unknown constant parameters, Theorems 3.2-3.4 remain true for a stationary nonlinear panel data setting. In this case, one will need only to change the estimation equation (2.7) to a semiparametric least-square (SLS) counterpart and then obtain a SLS estimator in (2.8) of Step 3 in Section 2. As a consequence, Theorem 3.1 will become an asymptotic normality for the SLS estimator.

Case 3: When both $F_{j t} \equiv 1$ and $\beta(\tau) \equiv \beta_{0}$ hold, model (1.1) reduces to a semiparametric cross-sectional (double-indexed) regression model. In this case, the corresponding results are of their own interest, and also generalize those for the semiparametric spatial data setting discussed in Gao et al. (2006), in which one could impose strict stationarity and $\alpha$-mixing condition on the spatial dataset. 


\section{Instruction for implementations}

For the convenience of users to apply the PMI method for real data analysis, we provide an instruction about how to implement the estimation procedure in practice.

\subsection{Construction of $\widehat{\boldsymbol{\beta}}(\tau), \widehat{\lambda}_{j}\left(v_{j}\right)$ and $\widehat{F}_{j t}$}

We first introduce the following notation:

$$
\begin{aligned}
& \mathbf{W}_{b}(\tau)=\operatorname{diag}\left(K\left(\frac{\tau_{1}-\tau}{b}\right), \ldots, K\left(\frac{\tau_{T}-\tau}{b}\right)\right) \\
& \mathbf{M}_{b}(\tau)=\left(\begin{array}{ccc}
1 & \ldots & 1 \\
\frac{\tau_{1}-\tau}{b} & \ldots & \frac{\tau_{T}-\tau}{b}
\end{array}\right)^{\top}, \quad \mathcal{V}_{i t}(\mathbf{v})=\left(\frac{V_{1 i t}-v_{1}}{h_{1}}, \frac{V_{2 i t}-v_{2}}{h_{2}}, \ldots, \frac{V_{J i t}-v_{J}}{h_{J}}\right)^{\top}, \\
& \mathcal{K}\left(\mathcal{V}_{i t}(\mathbf{v})\right)=\prod_{j=1}^{J} K\left(\frac{V_{j i t}-v_{j}}{h_{j}}\right), \quad \mathcal{W}_{t}(\mathbf{v})=\operatorname{diag}\left(\mathcal{K}\left(\mathcal{V}_{1 t}(\mathbf{v})\right), \ldots, \mathcal{K}\left(\mathcal{V}_{N t}(\mathbf{v})\right)\right), \\
& \mathcal{M}_{t}(\mathbf{v})=\left(\begin{array}{ccc}
1 & \ldots & 1 \\
\mathcal{V}_{1 t}(\mathbf{v}) & \ldots & \mathcal{V}_{N t}(\mathbf{v})
\end{array}\right)^{\top} .
\end{aligned}
$$

Then the main steps can be summarised as follows.

(1) Step 1: We can first compute $\widehat{x}_{i t}^{(l) *}$ and $\widehat{y}_{i t}^{*} \cdot m_{X, i}^{(l)}(\tau)$ and $m_{Y, i}(\tau)$ can be estimated by the local linear method:

$$
\begin{aligned}
& \widehat{m}_{X, i}^{(l)}(\tau)=[1,0]\left(\mathbf{M}_{b}^{\top}(\tau) \mathbf{W}_{b}(\tau) \mathbf{M}_{b}(\tau)\right)^{-1} \mathbf{M}_{b}^{\top}(\tau) \mathbf{W}_{b}(\tau) \mathbf{x}_{i .}^{(l)}, \\
& \widehat{m}_{Y, i}(\tau)=[1,0]\left(\mathbf{M}_{b}^{\top}(\tau) \mathbf{W}_{b}(\tau) \mathbf{M}_{b}(\tau)\right)^{-1} \mathbf{M}_{b}^{\top}(\tau) \mathbf{W}_{b}(\tau) \mathbf{y}_{i .} .
\end{aligned}
$$

for each $i$, where $\mathbf{x}_{i .}^{(l)}=\left(x_{i 1}^{(l)}, \cdots, x_{i T}^{(l)}\right)^{\top}$ and $\mathbf{y}_{i}=\left(y_{i 1}, \cdots, y_{i T}\right)^{\top}$. Therefore $\widehat{\widetilde{x}}_{i t}^{(l)}$ and $\widehat{\widetilde{y}}_{i t}$ can be computed as $\widehat{\widetilde{x}}_{i t}^{(l)}=x_{i t}^{(l)}-\widehat{m}_{X, i}^{(l)}\left(\tau_{t}\right)$ and $\widehat{\widetilde{y}}_{i t}=y_{i t}-\widehat{m}_{Y, i}\left(\tau_{t}\right)$. Then we define the estimators $\mathcal{H}_{N, t}^{(l)}(\mathbf{v})$ and $\mathcal{H}_{N, t}^{(0)}(\mathbf{v})$ as follows:

$$
\begin{aligned}
& \mathcal{H}_{N, t}^{(l)}(\mathbf{v})=\left[1, \mathbf{0}_{1 \times J}\right]\left(\mathcal{M}_{t}^{\top}(\mathbf{v}) \mathcal{W}_{t}(\mathbf{v}) \mathcal{M}_{t}(\mathbf{v})\right)^{-1} \mathcal{M}_{t}^{\top}(\mathbf{v}) \mathcal{W}_{t}(\mathbf{v}) \widehat{\widetilde{\mathbf{x}}}_{t}^{(l)} \\
& \mathcal{H}_{N, t}^{(0)}(\mathbf{v})=\left[1, \mathbf{0}_{1 \times J}\right]\left(\mathcal{M}_{t}^{\top}(\mathbf{v}) \mathcal{W}_{t}(\mathbf{v}) \mathcal{M}_{t}(\mathbf{v})\right)^{-1} \mathcal{M}_{t}^{\top}(\mathbf{v}) \mathcal{W}_{t}(\mathbf{v}) \widehat{\widetilde{\mathbf{y}}}_{\cdot t}
\end{aligned}
$$

for each $t$, where $\widehat{\widetilde{\mathbf{x}}}_{\cdot t}^{(l)}=\left(\widehat{\widetilde{x}}_{1 t}^{(l)}, \cdots, \widehat{\widetilde{x}}_{N t}^{(l)}\right)^{\top}$ and $\widehat{\widetilde{\mathbf{y}}}_{\cdot t}=\left(\widehat{\widetilde{y}}_{1 t}, \cdots, \widehat{\widetilde{y}}_{N t}\right)^{\top}$.

(2) Step 2: With the availability of $\mathcal{H}_{N, t}^{(l)}(\mathbf{v})$, the MI projection matrices can be constructed as

$$
\widehat{\mathcal{P}}_{j t}^{(l)}\left(v_{j}\right)=\frac{1}{N} \sum_{i=1}^{N} \mathcal{H}_{N, t}^{(l)}\left(\mathbf{V}_{i t}^{(-j)}\left(v_{j}\right)\right) W^{(-j)}\left(\mathbf{V}_{i t}^{(-j)}\right) w_{j}\left(v_{j}\right),
$$

for $l=0,1, \ldots, p$.

We can then compute $\widehat{x}_{i t}^{(l) *}$ and $\widehat{y}_{i t}^{*}$ by $\widehat{x}_{i t}^{(l) *}=\widehat{\widetilde{x}}_{i t}^{(l)}-\sum_{j=1}^{J} \widehat{\mathcal{P}}_{j t}^{(l)}\left(V_{j i t}\right)$ and $\widehat{y}_{i t}^{*}=\widehat{\widetilde{y}}_{i t}-$ $\sum_{j=1}^{J} \widehat{\mathcal{P}}_{j t}^{(0)}\left(V_{j i t}\right)$. 
(3) Step 3: With $\widehat{x}_{i t}^{(l) *}$ and $\widehat{y}_{i t}^{*}$, we construct $\widehat{\boldsymbol{\beta}}(\tau)$ by the local linear method as described in (2.6).

(4) Step 4: With $\widehat{\boldsymbol{\beta}}(\tau)$, we finally compute $\widehat{\lambda}_{j}\left(v_{j}\right)$ and $\widehat{F}_{j t}$ as in (2.10) and (2.11), respectively.

\subsection{Choice of kernel functions and bandwidths}

In applications, many conventional kernel functions that satisfy Assumption 3.(3) can be used in the local linear estimation. In terms of the empirical studies in Section 5 below, the Epanechnikov kernel function $K(\mu)=\frac{3}{4}\left(1-\mu^{2}\right) I(|\mu| \leq 1)$ and Gaussian kernel are used in the nonparametric estimation of $\boldsymbol{\beta}(\tau)$ and $\lambda\left(v_{j}\right)$, respectively.

The bandwidth can be selected by the following leave-one-unit-out cross-validation method:

$$
h_{c v}=\min _{h} \sum_{t=1}^{T} \sum_{i=1}^{N}\left(y_{i t}-\mathbf{x}_{i t}^{\top} \widehat{\boldsymbol{\beta}}^{(-i)}\left(\tau_{t}\right)-\sum_{j=1}^{J} \widehat{\lambda}_{j}^{(-i)}\left(V_{j i t}\right) \widehat{F}_{j t}^{(-i)}\right)^{2},
$$

where $\left(\widehat{\boldsymbol{\beta}}^{(-i)}(\tau), \widehat{\lambda}_{j}^{(-i)}\left(v_{j}\right), \widehat{F}_{j t}^{(-i)}\right)$ are leave-one-unit-out estimators, which can be derived without using the observations of individual $i$.

\subsection{Construction of confidence intervals}

Recall that the $\alpha$-mixing conditions in Assumption 2 are imposed on the stationary random variables. The classic wild bootstrap method may not be valid due to the existence of possible serial correlation. To address this issue, the moving blocks bootstrap (MBB) method proposed by Gonçalves (2011) is employed in our study. Gonçalves (2011) shows that the MBB method is robust to serial correlation and cross-sectional dependence therefore it is consistent with our theoretical setup in Section 3. The details of MBB procedure are summarized as follows:

(1) Obtain the PMI estimators: $\widehat{\boldsymbol{\beta}}(\tau), \widehat{\lambda}_{j}\left(v_{j}\right)$ and $\widehat{F}_{j t}$.

(2) Calculate the residuals:

$$
\widehat{\varepsilon}_{i t}=y_{i t}-\mathbf{x}_{i t}^{\top} \widehat{\boldsymbol{\beta}}\left(\tau_{t}\right)+\sum_{j=1}^{J} \widehat{\lambda}_{j}\left(V_{j i t}\right) \widehat{F}_{j t}
$$

(3) For some pre-chosen value $l_{0}$, let $k_{0}=\left[T / l_{0}\right]+1$. Resample $\left\{l_{1}, l_{2}, \ldots, l_{k_{0}}\right\}$ from $\{1,2, \ldots, T-$ $\left.l_{0}\right\}$. Then we construct the bootstrapping residuals by: $\widehat{\varepsilon}_{i 1}^{*}=\widehat{\varepsilon}_{i l_{1}}, \ldots, \widehat{\varepsilon}_{i l_{0}}^{*}=\widehat{\varepsilon}_{i, l_{1}+l_{0}-1}, \ldots$, $\widehat{\varepsilon}_{i, m l_{0}+1}^{*}=\widehat{\varepsilon}_{i l_{m}}, \ldots, \widehat{\varepsilon}_{i,(m+1) l_{0}}^{*}=\widehat{\varepsilon}_{i, l_{m}+l_{0}-1}, \ldots, \widehat{\varepsilon}_{i,\left(k_{0}-1\right) l_{0}+1}^{*}=\widehat{\varepsilon}_{i l_{k_{0}}}, \ldots, \widehat{\varepsilon}_{i T}^{*}=\widehat{\varepsilon}_{i, l_{k_{0}}+T-\left(k_{0}-1\right) l_{0}-}$ 1. In each replication, the bootstrapping sample is obtained by $\widehat{y}_{i t}^{*}=\widehat{y}_{i t}+\widehat{\varepsilon}_{i t}^{*}$, where $\varepsilon_{i t}^{*}=\widehat{\varepsilon}_{i k}$.

(4) With the observations $\left\{\widehat{y}_{i t}^{*}, x_{1, i t}, x_{1, i t}, V_{1 i t}, V_{2 i t}\right\}$, we can re-estimate the unknown functions by the PMI method.

(5) Repeat Steps 3 and 4 for $R=1000$ times and calculate the bootstrap confidence intervals. 


\subsection{Out-of-sample forecasting procedure}

In this section, we provide a detailed description of the main steps to obtain out-of-sample forecasts from the PMI estimates. Since the loading function $\widehat{\lambda}_{j}\left(v_{j}\right)$ is independent of time $t$, the main task here is to construct out-of-sample forecasts for $\widehat{\beta}(\tau)$ and $\widehat{F}_{j t}$.

(1) Obtain the PMI estimators: $\widehat{\boldsymbol{\beta}}\left(t / T_{0}\right), \widehat{\lambda}_{j}\left(v_{j}\right)$ and $\widehat{F}_{j t}$ for $t=1,2, \ldots, T_{0}$.

(2) We then conduct a parametric calibration for the nonparametrically estimated function $\widehat{\boldsymbol{\beta}}(\tau)$ :

$$
\widehat{\boldsymbol{\beta}}\left(t / T_{0}\right)=\theta_{0}+\theta_{1}\left(t / T_{0}\right)+\theta_{2}\left(t / T_{0}\right)^{2}+\cdots+\theta_{l}\left(t / T_{0}\right)^{l}+u\left(t / T_{0}\right)
$$

where $l$ is a pre-determined positive integer and $u\left(t / T_{0}\right)$ is the residual function. Then we can obtain the estimates $\left\{\widehat{\theta}_{0}, \widehat{\theta}_{1}, \ldots, \widehat{\theta}_{l}\right\}$ by OLS method. Then the $n_{0}$-step-ahead out-ofsample forecast of $\widehat{\boldsymbol{\beta}}(\cdot)$ is given by

$$
\widetilde{\boldsymbol{\beta}}_{T_{0}+n_{0}}=\widehat{\theta}_{0}+\widehat{\theta}_{1}\left(\left(T_{0}+n_{0}\right) / T_{0}\right)+\widehat{\theta}_{2}\left(\left(T_{0}+n_{0}\right) / T_{0}\right)^{2}+\cdots+\widehat{\theta}_{l}\left(\left(T_{0}+n_{0}\right) / T_{0}\right)^{l} .
$$

(3) For the factor estimates, we obtain the $n_{0}$-step-ahead out-of-sample forecast $\widetilde{F}_{j, T_{0}+n_{0}}$ by fitting a univariate $\operatorname{ARMA}(p, q)$ model where $p$ and $q$ are a pre-determined positive integers.

(4) We finally construct the $n_{0}$-step-ahead out-of-sample forecast $\widetilde{y}_{i, T_{0}+n_{0}}$ as follows:

$$
\widetilde{y}_{i, T_{0}+n_{0}}=\mathbf{x}_{i, T_{0}+n_{0}}^{\top} \widetilde{\boldsymbol{\beta}}_{T_{0}+n+0}+\sum_{j=1}^{J} \widehat{\lambda}_{j}\left(V_{j i, T_{0}+n_{0}}\right) \widetilde{F}_{j, T_{0}+n_{0}} .
$$

In addition, we calculate the MSE of the $n_{0}$-step-ahead out-of-sample forecast errors:

$$
\operatorname{MSE}_{T_{0}}\left(n_{0}\right)=\frac{1}{N} \sum_{i=1}^{N}\left(\widetilde{y}_{i, T_{0}+n_{0}}-y_{i, T_{0}+n_{0}}\right)^{2} .
$$

In the following section, we examine the finite-sample performance of the proposed model and method using two empirical examples.

\section{$5 \quad$ Empirical studies}

In this section, we provide two empirical studies to illustrate the finite sample performance of the proposed PMI estimation. In Section 5.1, we study the security characteristics predicting stock returns when investor sentiment effect is considered. In Section 5.2, the determinants of health care expenditure in OECD countries are examined and the income elasticity of health care expenditure is estimated. 


\subsection{An empirical analysis on US stock market}

The relationship between individual stock returns and security characteristics has been well studied since the FamaFrench factor models were proposed (see Fama and French, 1992, 1993), who first used size and value factors to model excess returns. More recently, momentum and own-volatility have been proved to be important as well in explaining return comovements (see Ang et al., 2006; Connor et al., 2012; Fan et al., 2016). These studies concentrate on the the firm characteristics without considering the effects of other variables on stock returns, such as investor sentiment effect. Baker and Wurgler (2006) reveal that the effects of security characteristics on stock returns is related to investor sentiment in US stock market. In terms of methodology, Baker and Wurgler (2006) use the following parametric model to capture the relationship between excess stock returns $\left(E r_{i t}\right)$, investor sentiment effect $\left(\mathrm{ISE}_{t}\right)$ and firm characteristics $\left(\mathbf{V}_{i t}=\right.$ $\left.\left(V_{1 i t}, \ldots, V_{J i t}\right)^{\top}\right)$ :

$$
E r_{i t}=\mathbf{x}_{i, t-1}^{\top} \boldsymbol{\beta}+\mathbf{V}_{i, t-1}^{\top} \boldsymbol{\gamma}+\varepsilon_{i t}
$$

where $\mathbf{x}_{i t}=\operatorname{ISE}_{t} \mathbf{V}_{i t}$. Since the coefficients $(\boldsymbol{\beta}$ and $\boldsymbol{\gamma})$ in Model (5.1) are assumed to be unknown parameters, Baker and Wurgler (2006) suggest the firm characteristics and investor sentiment can jointly affect the stock returns in a static and known form.

In this section, we re-examine the investor sentiment effect and four widely-used firm characteristics (size, value, momentum and own volatility) on a US stock return dataset. The dataset used in this section is from the Center for Research in Security Prices (CRSP), which includes the complete monthly return records for 164 non-financial S\&P 500 companies from 2000 to 2018 ( $N=164$ and $T=228)$. We obtain the observations of market capitalisation and book value from Compustat and then construct four characteristicssize $\left(\operatorname{Size}_{i t}\right)$, value $\left(\right.$ Value $\left._{i t}\right)$, momentum $\left(\mathrm{Mom}_{i t}\right)$ and volatility $\left(\mathrm{Vol}_{i t}\right)$-in the same method as Connor et al. (2012) and Fan et al. (2016). In detail, the raw size characteristic for each security is the log version of the market value on the last trade day in the previous month. The raw value equals the ratio of the market value on the previous trade day to the book value in the previous month. The raw momentum characteristic is derived as the cumulative return from the previous 6 months. Last, the raw volatility is computed as the standard deviation of the security returns in the previous 6 months. All four characteristics are then standardised to have zero mean and unit variance. We calculate daily returns in excess of risk-free rates $\left(\operatorname{Er}_{i t}\right)$, where the risk-free rate data are collected from CRSP. In terms of the investor sentiment effect, the literature uses various measures or observable proxies, such as average closed-end fund discount, mutual fund flows and IPO-based indicator variables. Baker and Wurgler (2006) use the principal components analysis method to construct an index by aggregating five separate proxies: the average closed-end fund discount, NYSE share turnover, the number and average first-day returns on IPOs, the equity share in new issues, and the dividend premium. This index has been widely accepted by the literature as a good indicator for investor sentiment effect since it is proposed (see Baker et al., 2012; Stambaugh et al., 2012, for example). In this section, we use Baker and Wurgler (2006)'s 
index as the proxy of investor sentiment effect $\left(\mathrm{ISE}_{t}\right)$.

Table 1: IPS unit root test results for characteristic variables

\begin{tabular}{ccccccc}
\hline Variables & & $W_{\text {tbar }}$ & $p$-value & & $Z_{\text {tbar }}$ & $p$-value \\
\cline { 1 - 1 } \cline { 6 - 7 } Size $_{i t}$ & & -6.43 & $<0.01$ & & -6.37 & $<0.01$ \\
Value $_{i t}$ & & -28.28 & $<0.01$ & & -28.42 & $<0.01$ \\
Mom $_{i t}$ & & -37.30 & $<0.01$ & & -37.90 & $<0.01$ \\
Vol $_{i t}$ & & -38.15 & $<0.01$ & & -38.27 & $<0.01$ \\
\hline
\end{tabular}

To examine the validity of model (1.1) for this data, we conduct unit root tests on the four firm characteristics and investor sentiment index. The well-known panel data IPS test proposed by Im et al. (2003) is used to detect potential unit roots of these characteristic variables and the results are reported in Table 1. The IPS test statistics $W_{t b a r}$ and $Z_{t b a r}$ are defined by Equation (4.10) and (4.6) by Im et al. (2003), respectively. As can be seen in Table 1, we don't have sufficient evidence to accept the null of panel unit root for these variables. For the time series variable ISE $_{t}$, we conduct the ADF unit root test with intercept that suggests $t-$ statistics $=-4.35$ and the rejection of nonstationarity at $1 \%$ confidence level. Therefore, the validity of the stationarity assumption is confirmed by the unit root test results and nonparametric detrending of the regressors is not necessary for this data set.

We then estimate characteristics beta-functions in the following additive model:

$$
\begin{aligned}
\operatorname{Er}_{i t}= & \sum_{k=1}^{4} x_{k, i, t-1} \beta_{k}\left(\tau_{t}\right)+\lambda_{1}\left(\operatorname{Size}_{i, t-1}\right) F_{1 t}+\lambda_{2}\left(\text { Value }_{i, t-1}\right) F_{2 t}+\lambda_{3}\left(\operatorname{Mom}_{i, t-1}\right) F_{3 t} \\
& +\lambda_{4}\left(\operatorname{Vol}_{i, t-1}\right) F_{4 t}+\varepsilon_{i t},
\end{aligned}
$$

where $x_{1, i, t} \equiv x_{1, i t}=$ Size $_{i t} \mathrm{ISE}_{t}, x_{2, i, t} \equiv x_{2, i t}=$ Value $_{i t} \mathrm{ISE}_{t}, x_{3, i, t} \equiv x_{3, i t}=\mathrm{Mom}_{i t} \mathrm{ISE}_{t}$ and $x_{4, i, t} \equiv x_{4, i t}=\operatorname{Vol}_{i t} \mathrm{ISE}_{t}$. Model (5.2) generalises Model (5.1) proposed by Baker and Wurgler (2006) by relaxing the pre-specified functional form of the characteristic betas and investor sentiment effect.

The Epanechnikov kernel function and Gaussian kernel are used in the nonparametric estimation of time-varying functions and characteristics beta-functions, respectively. The bandwidth is selected by the leave-one-unit-out cross-validation method as discussed in Section 4 . The estimated time-varying regression coefficients are reported in Figure 1. The $90 \%$ confidence intervals are computed through MBB method proposed by Gonçalves (2011) as described in details in Section 4. As can be seen from Figure 1, the estimated functions for $x_{1, i t}, x_{3, i t}$ and $x_{4, i t}$ are significantly different from the zero line and the estimated curves exhibit evident nonlinear patterns. For example, $\beta_{3}(\tau)$ increases from 0 to 0.025 before it drops to -0.05 and then fluctuates around -0.025 . These estimation results imply that the investor sentiment effect may affect the stock returns in certain periods and its effect is reflected mainly through the size momentum and volatility variables. As a comparison, we calculate parametric estimates from model (5.1), which are reported in Figure 1 as constant lines. The parametric estimates are 

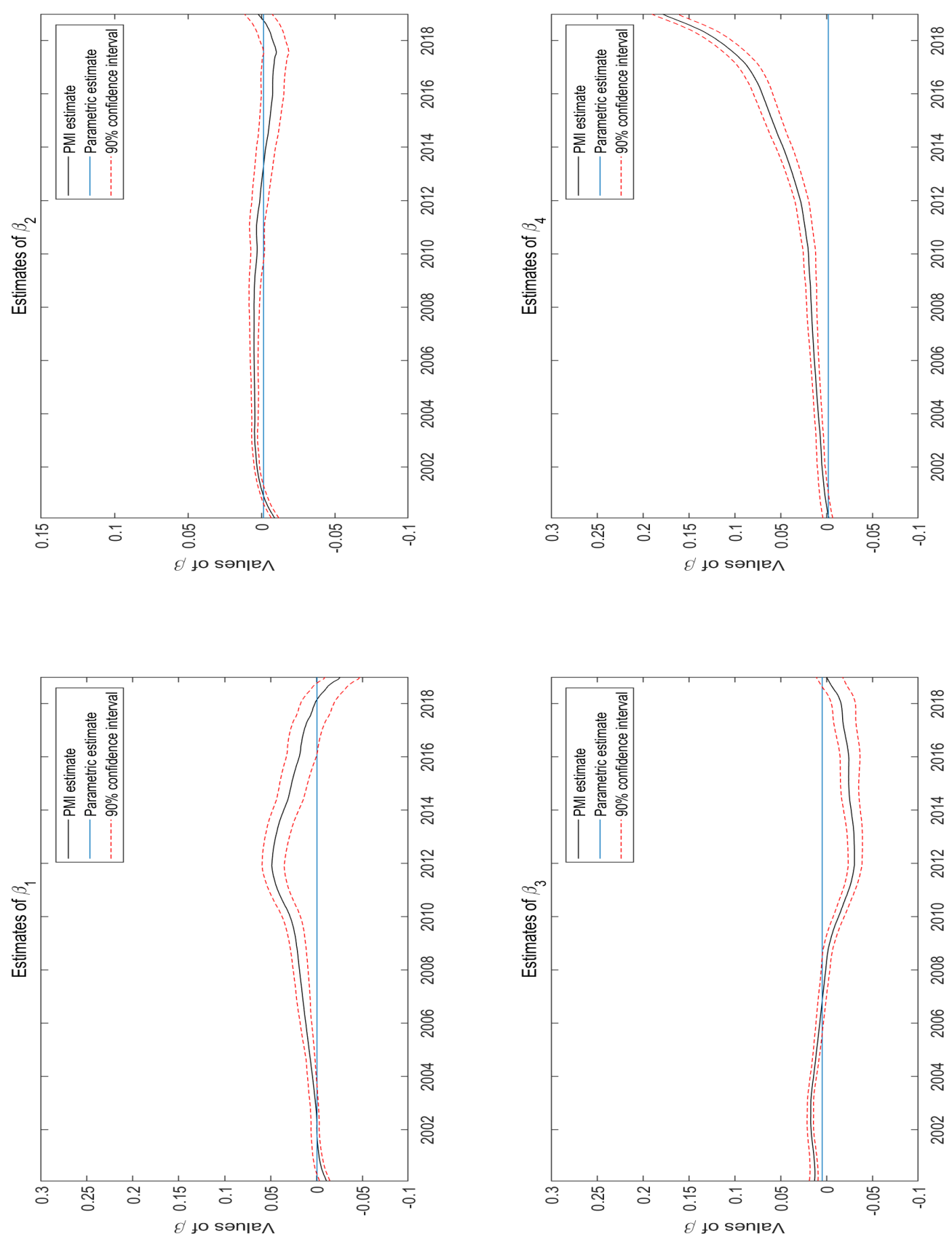

Figure 1: Estimated time-varying regression coefficient functions for Model 5.2 

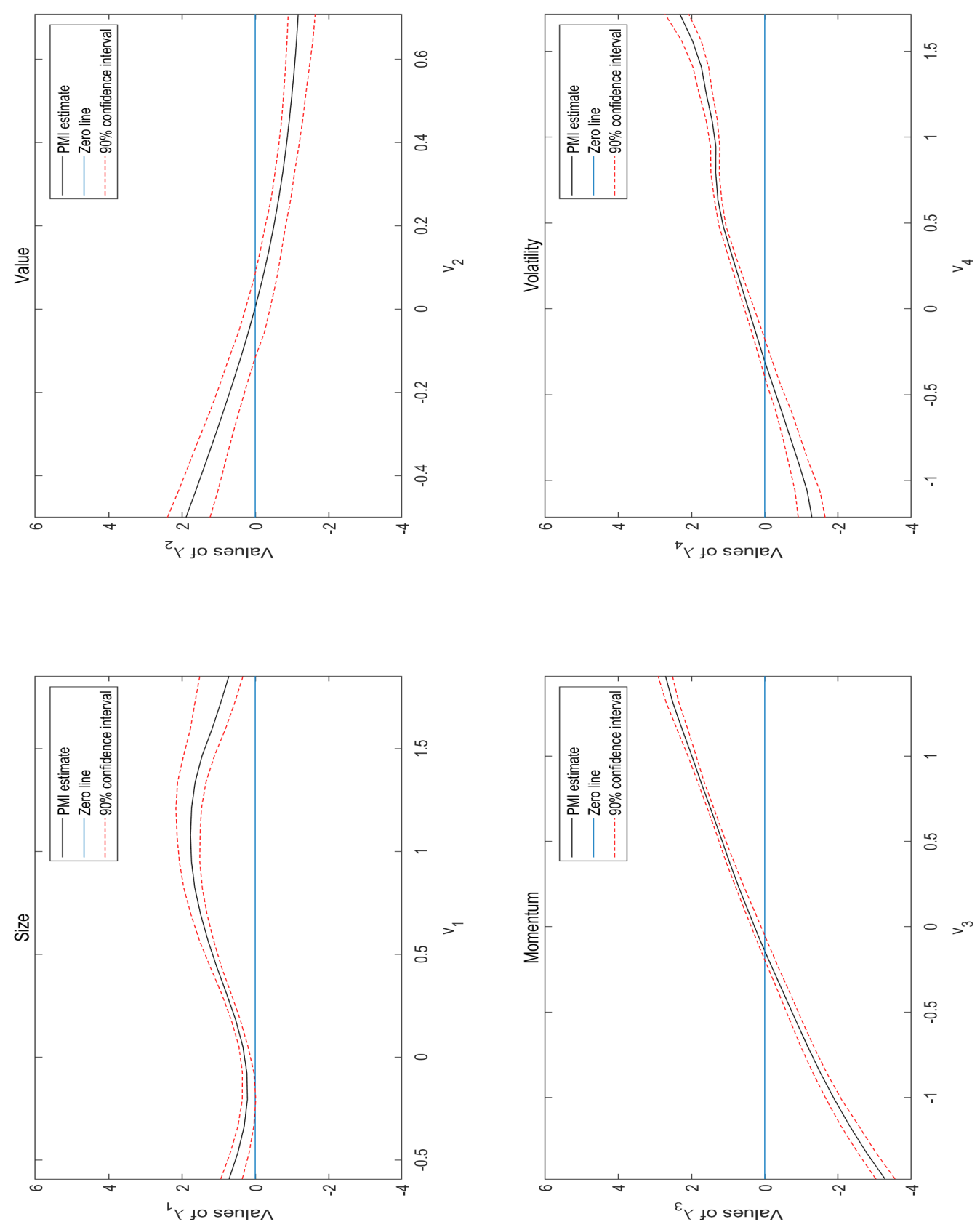

Figure 2: Estimated loading functions for Model 5.2 
$-0.0007,-0.0015,0.0046$ and -0.0020 , respectively. The corresponding $p$-values of $t$-test are 0.2690, 0.0162, $7.5873 \times e^{-13}$ and 0.0017. By comparing the PMI estimates with their parametric counterparts, we can conclude that the time variations in these coefficients are significant.

Figure 2 displays the PMI estimates of the loading functions in Model (5.2), which suggests that all estimated loading functions are significant according to the bootstrap confidence intervals even when the investor sentiment effect is considered. It indicates that these four characteristic variables have important roles in explaining the cross-sectional and time serial differences in US stock returns. In addition, all other three functions except momentum show clear nonlinear patterns. The size function fluctuates between 0 and 2 as the value of size goes up. The value function decreases gradually from around 2 to below -1 when the value characteristic goes up from -0.6 to 0.4. Both momentum and volatility functions increase significantly while the volatility characteristic function exhibits more nonlinear patterns when the value of volatility is above 0 .

To examine the out-of-sample forecasting performance of the proposed PMI method, we use the observations of manufacturing companies in the first 10 years to construct estimators and then compute the 5, 10, and 15-step-ahead forecasting errors. For the computational simplicity we assume the factors are constant over time. The MSEs for the PMI approach and parametric methods are reported in Table 2. As can be seen from Table 2, the proposed PMI method outperforms the parametric model in terms of the 5, 10 and 15-step-ahead out-of-sample forecasts.

Table 2: MSEs of the out-of-sample forecast errors

\begin{tabular}{ccccc}
\hline Estimator & & \multicolumn{3}{c}{ No. of steps ahead } \\
\cline { 5 - 6 } \cline { 5 - 6 } PMI & & 0.0048 & 0.0104 & 0.0017 \\
Parametric & & 0.0053 & 0.0106 & 0.0022 \\
\hline
\end{tabular}

To summarise the estimation results from this empirical study, we first confirm the explanatory power of the size, value, momentum and volatility in modelling stock returns in US stock market, even when the investor sentiment effect is considered. Secondly, the investor sentiment effect also affects stock returns significantly and this effect is captured mainly by the momentum and volatility characteristics. Finally, nonlinear patterns are revealed in both loading functions and regression coefficient functions according to our estimation.

\subsection{An empirical study on OECD health care expenditure}

The determinants of health care expenditure in OECD countries have been well studied in the literature. Among these determinants, income has been identified as the most important factor explaining health care expenditure diversities (see Jones and Wildman, 2008; Baltagi and Moscone, 2010, for example). In this section, we re-estimate the income elasticity in OECD countries using the proposed PMI method. Our analysis uses an annual data set from 1972 to 
$2013(T=42)$ on 18 OECD countries $(N=18)$. In addition to GDP (GDP $i t$ : per capita in US dollars), the proportions of population aged below 14 and above 65 over all population ( $\mathrm{DR}_{i t}^{\text {young }}$ and $\mathrm{DR}_{i t}^{\text {old }}$, respectively) and the government health care expenditure (GHE $\mathrm{G}_{i t}$ : public funding proportion in total spending) are used as observable determinants of health care expenditure ( $\mathrm{HE}_{i t}$ : per capita in US dollars). All variables are expressed in natural logarithm. In addition to these level variables, Hauck and Zhang (2016) show that the growth of determinants may also explain the differences in health care expenditure. Therefore, we introduce a factor structure with the the growth variables of three determinants ${ }^{1}\left(\Delta \mathrm{GDP}_{i t}, \Delta \mathrm{DR}_{i t}^{\text {old }}\right.$ and $\left.\Delta \mathrm{GHE}_{i t}\right)$ as the observable covariates in the loading functions.

Table 3: IPS unit root test results for regressor residuals and loading variables

\begin{tabular}{|c|c|c|c|c|}
\hline Residuals in Regressors & $W_{t b a r}$ & $p$-value & $Z_{t b a r}$ & $p$-value \\
\hline $\mathrm{GDP}_{i t}$ & -4.30 & $<0.01$ & -4.32 & $<0.01$ \\
\hline $\mathrm{DR}_{i t}^{\text {young }}$ & -9.56 & $<0.01$ & -9.77 & $<0.01$ \\
\hline $\mathrm{DR}_{i t}^{\text {old }}$ & -6.25 & $<0.01$ & -6.34 & $<0.01$ \\
\hline $\mathrm{GHE}_{i t}$ & -5.61 & $<0.01$ & -5.64 & $<0.01$ \\
\hline Loading variables & $W_{t b a r}$ & $p$-value & $Z_{t b a r}$ & $p$-value \\
\hline$\Delta \mathrm{GDP}_{i t}$ & -19.31 & $<0.01$ & -19.37 & $<0.01$ \\
\hline$\Delta \mathrm{DR}_{i t}^{\text {old }}$ & -15.60 & $<0.01$ & -15.77 & $<0.01$ \\
\hline$\Delta \mathrm{GHE}_{i t}$ & -19.25 & $<0.01$ & -19.31 & $<0.01$ \\
\hline
\end{tabular}

As described in Section 2, the nonstationary patterns in regressors are allowed and they can be removed by nonparametric detrending. To examine the validity of this assumption, we first estimate the unknown trends in regressors by the local linear method to obtain the residuals, and then conduct unit root tests on these residuals. The well-known IPS test proposed by Im et al. (2003) is conducted in this section to confirm the stationarity of the residuals and the results are reported in Table 3. The IPS test statistics $W_{t b a r}$ and $Z_{t b a r}$ are defined by Equation (4.10) and (4.6) by Im et al. (2003), respectively. Table 1 shows that the residuals after detrending are stationary for these four determinant variables. In term of the growth variables, IPS test results are reported in Table 3 which can also reject the null of panel unit root. Therefore, we confirm that PMI method is suitable for this health care expenditure dataset. We consider the following model:

$$
\begin{aligned}
\mathrm{HE}_{i t}= & \beta_{1}\left(\tau_{t}\right) \mathrm{GDP}_{i t}+\beta_{2}\left(\tau_{t}\right) \mathrm{DR}_{i t}^{\text {young }}+\beta_{3}\left(\tau_{t}\right) \mathrm{DR}_{i t}^{\text {old }}+\beta_{4}\left(\tau_{t}\right) \mathrm{GHE}_{i t}+\lambda_{1}\left(\Delta \mathrm{GDP}_{i t}\right) F_{1 t} \\
& +\lambda_{2}\left(\Delta \mathrm{DR}_{i t}^{\text {old }}\right) F_{2 t}+\lambda_{3}\left(\Delta \mathrm{GHE}_{i t}\right) F_{3 t}+\varepsilon_{i t} .
\end{aligned}
$$

In this section, we continue using the Epanechnikov kernel function and Gaussian kernel in the nonparametric estimation of time-varying functions and loading functions, respectively. The

\footnotetext{
${ }^{1}$ Initially, we use growth variables of all these four determinants as observable loading covariates. Since the loading function for $\Delta \mathrm{DR}_{i t}^{\text {young }}$ is insignificant, it is then removed from the proposed model.
} 

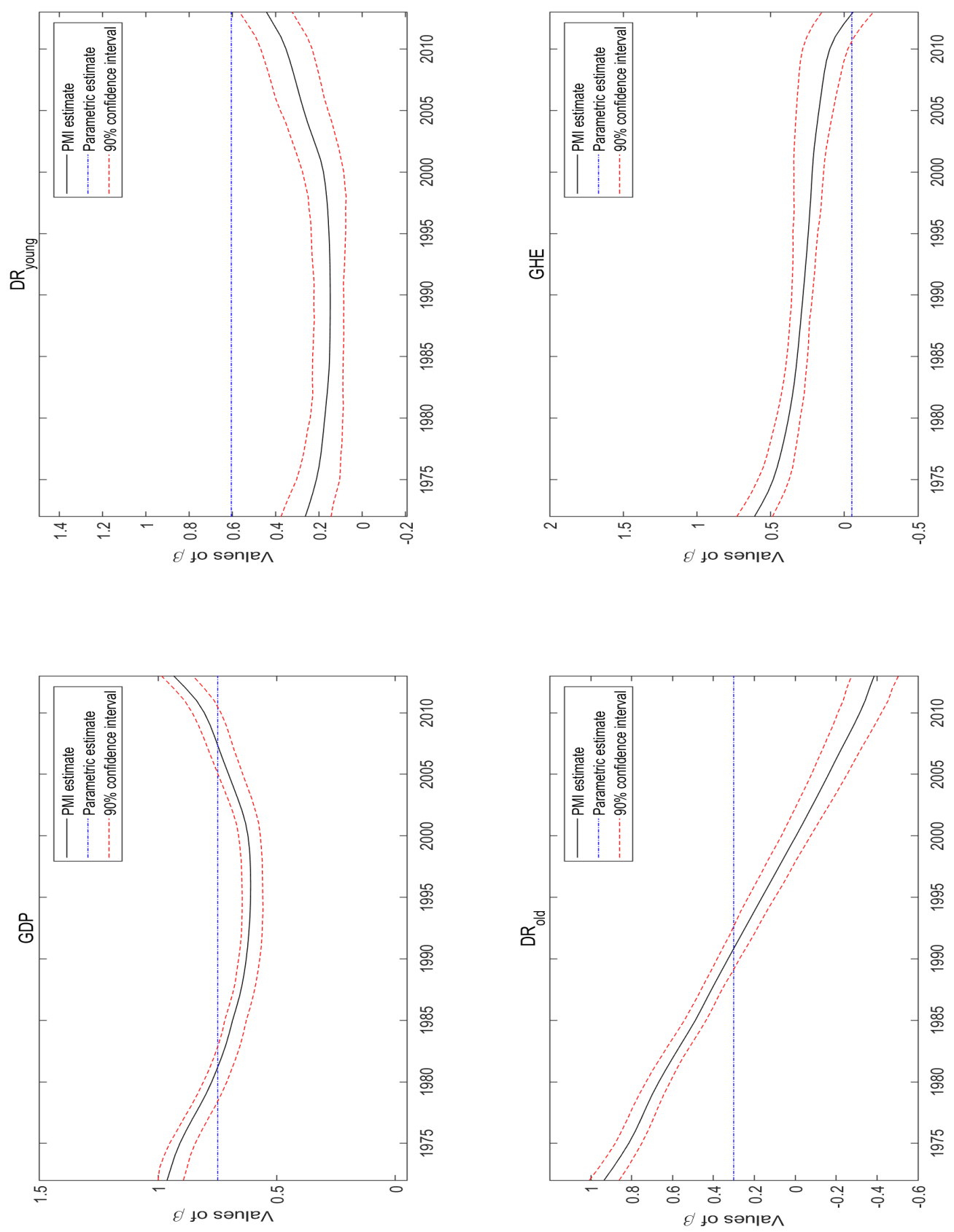

Figure 3: Estimated time-varying regression coefficient functions for Model 5.3 

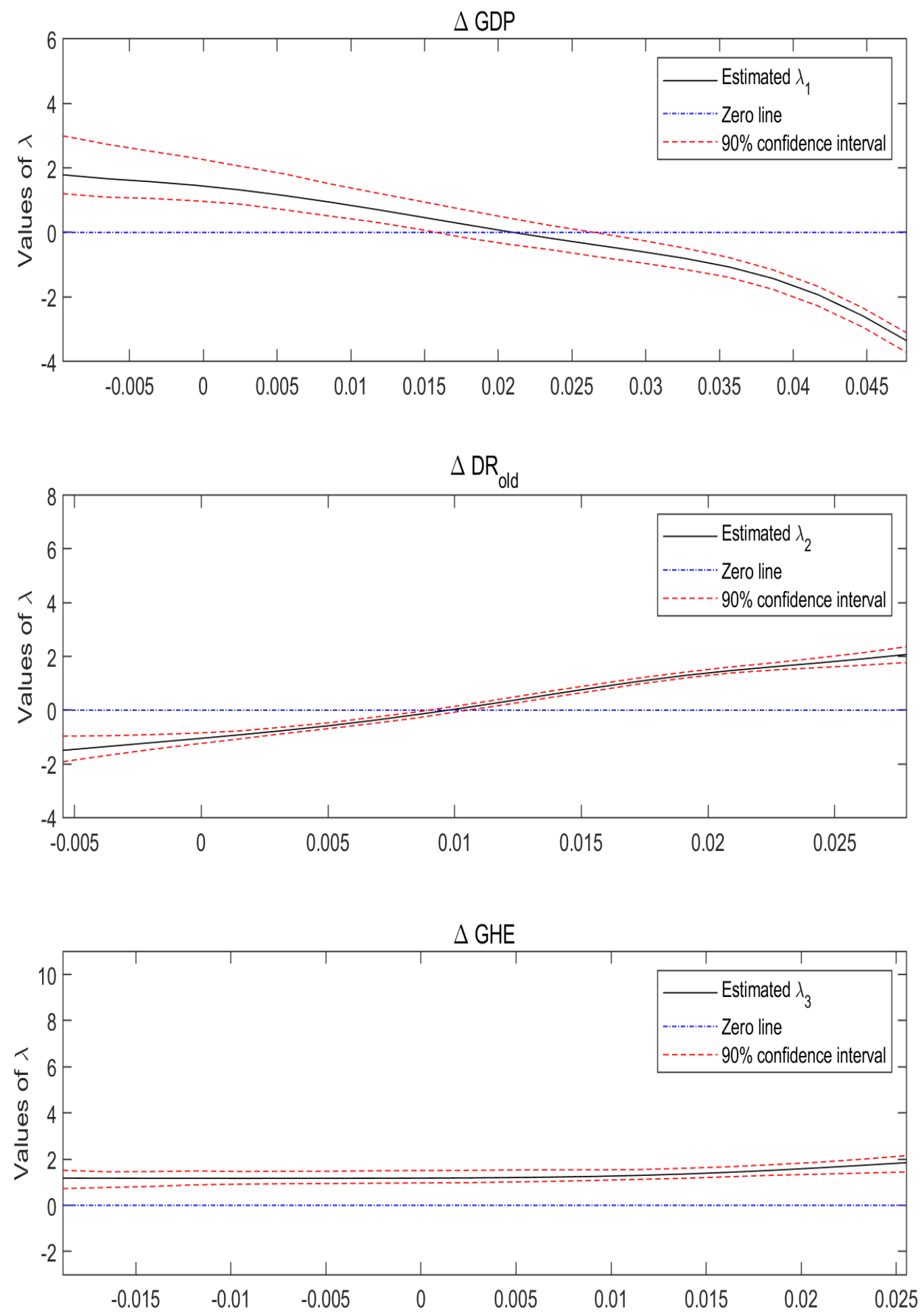

Figure 4: Estimated loading functions for Model 5.3 
bandwidth is selected by the leave-one-unit-out cross-validation method. We employ the PMI approach proposed in Section 2 to obtain consistent estimation and Gonçalves (2011)'s MBB method to construct $90 \%$ confidence intervals for the PMI estimators.

Table 4: MSEs of the out-of-sample forecast errors (health expenditure data)

\begin{tabular}{ccccc}
\hline Estimator & & \multicolumn{3}{c}{ No. of steps ahead } \\
\cline { 1 - 3 } \cline { 4 - 5 } PMI & & 0.0497 & 0.0741 & 0.0572 \\
Parametric & & 0.0444 & 0.0835 & 0.1567 \\
\hline
\end{tabular}

The PMI estimates of the beta functions are reported in Figure 3. As shown in Figure 3, the effects of these four determinants on health care expenditure exhibit time-variant features. According to our estimation results, income elasticity increases from around 0.5 to 0.8 in this period. Its $90 \%$ confidence interval is below 1 at any point which implies that the health-care service is a necessary good in OECD countries. The estimated loading functions are reported in Figure 4. The $90 \%$ confidence intervals suggest the overall significance of all these four loading functions, which confirms the necessity to include the growth variables when we model the diversities of health care expenditure. As a comparison, we obtain the parametric estimates of these elasticities by using the linear panel data model with interactive fixed effects proposed by Bai (2009). The parametric estimation results are reported in Figure 3. As can be seen from Figure 3, none of these parametric estimates can be covered entirely by the nonparametric estimation confidence intervals, which suggests the existence of time-variation in these four elasticities. In addition to the in-sample estimation, we also compute the out-of-sample forecast using model (1.1). The sample size used in estimating the models is 25 and the MSE of 5, 10 15 steps-ahead out-of-sample forecasting errors are reported in Table 4, which shows that the proposed PMI estimation method associated with model (1.1) outperforms the linear panel data model in terms of the 10 and 15-step-ahead out-of-sample forecasts.

\section{Simulation studies}

In this section, simulation experiments are conducted to assess the performance of our estimators with a small $N$ and $T$. We consider two DGPs. In the first example, we consider the fully exogenous loadings to accommodate the case where the explanatory variables are not correlated with the factor structure. In the second example, we allow that the regressors and loadings are dependent on each other.

Example 6.1 We consider the following DGP:

$$
y_{i t}=x_{1, i t} \beta_{1}\left(\tau_{t}\right)+x_{2, i t} \beta_{2}\left(\tau_{t}\right)+\lambda_{1}\left(V_{1, i t}\right) F_{1 t}+\lambda_{2}\left(V_{2, i t}\right) F_{2 t}+\varepsilon_{i t},
$$

where $\left(V_{1,1 t}, \ldots, V_{1, N t}\right)^{\top},\left(V_{2,1 t}, \ldots, V_{2, N t}\right)^{\top},\left(\eta_{1,1 t}, \ldots, \eta_{1, N t}\right)^{\top},\left(\eta_{2,1 t}, \ldots, \eta_{2, N t}\right)^{\top}$ and $\left(\varepsilon_{1 t}, \varepsilon_{2 t}, \ldots, \varepsilon_{N t}\right)^{\top}$ are generated as $N$-dimensional vectors of independent Gaussian variables with zero mean and 
covariance matrix: $\sigma_{i j}=0.8^{|i-j|}$, and $\left(\beta_{1}(\tau), \beta_{2}(\tau)\right)=(\sin (\pi \tau / 4), \cos (\pi \tau / 3)),\left(x_{1, i t}, x_{2, i t}\right)=$ $\left(\tau_{t}^{1 / 3}, \tau_{t}^{2 / 3}\right)+\left(\eta_{1, i t}, \eta_{2, i t}\right),\left(\lambda_{1}\left(v_{1}\right), \lambda_{2}\left(v_{2}\right)\right)=\left(v_{1}, v_{2}^{3} / \sqrt{15}\right)$ and $\left(F_{1 t}, F_{2 t}\right)=\left(\tau_{t},-\ln \tau_{t}\right)$.

Example 6.2 We consider the case where

$$
x_{1, i t}=\sqrt{\lambda_{1}\left(V_{1, i t}\right)^{2}+\lambda_{1}\left(V_{2, i t}\right)^{2}}+\eta_{1, i t} \text { and } x_{2, i t}=\lambda_{1}\left(V_{1, i t}\right)^{2}+\lambda_{1}\left(V_{2, i t}\right)^{2}+\eta_{2, i t} .
$$

All other variables are generated in the same way as Example 6.1.

In each estimation, the Epanechnikov kernel function $K(\mu)=\frac{3}{4}\left(1-\mu^{2}\right) I(|\mu| \leq 1)$ and Gaussian kernel are used in the local linear estimation of $\boldsymbol{\beta}(\tau)$ and $\lambda\left(v_{j}\right)$, respectively. The bandwidth is selected by the following leave-one-unit-out cross-validation method:

$$
h_{c v}=\min _{h} \sum_{t=1}^{T} \sum_{i=1}^{N}\left(y_{i t}-x_{1, i t} \widehat{\beta}_{1}^{(-i)}\left(\tau_{t}\right)-x_{2, i t} \widehat{\beta}_{2}^{(-i)}\left(\tau_{t}\right)-\widehat{\lambda}_{1}^{(-i)}\left(V_{1 i t}\right) \widehat{F}_{1 t}^{(-i)}+\widehat{\lambda}_{2}^{(-i)}\left(V_{2 i t}\right) \widehat{F}_{2 t}^{(-i)}\right)^{2},
$$

where $\left(\widehat{\beta}_{1}^{(-i)}(\tau), \widehat{\beta}_{2}^{(-i)}(\tau), \widehat{\lambda}_{1}^{(-i)}\left(v_{1}\right), \widehat{\lambda}_{1}^{(-i)}\left(v_{1}\right), \widehat{F}_{1 t}^{(-i)}, \widehat{F}_{2 t}^{(-i)}\right)$ are the leave-one-unit-out estimators, which are computed without using the observations of the individual $i$-th unit.

We replicate the experiments 1000 times $(R=1000)$ and calculate the root-average of mean squared errors (RMSEs) for $\left(\beta_{1}(\tau), \beta_{2}(\tau)\right),\left(\lambda_{1}\left(v_{1}\right), \lambda_{2}\left(v_{2}\right)\right)$ and $\left(F_{1 t}, F_{2 t}\right)$, respectively:

$$
\begin{aligned}
\operatorname{RMSE}_{\widehat{\beta}} & =\frac{1}{R} \sum_{r=1}^{R} \sqrt{\frac{1}{T} \sum_{t=1}^{T} \sum_{l=1}^{2}\left|\widehat{\beta}_{l}^{(r)}\left(\tau_{t}\right)-\beta_{l}\left(\tau_{t}\right)\right|^{2}}, \quad \operatorname{RMSE}_{\lambda}=\frac{1}{R} \sum_{r=1}^{R} \sqrt{\frac{1}{N T} \sum_{i=1}^{N} \sum_{t=1}^{T} \sum_{j=1}^{2}\left|\widehat{\lambda}_{j}^{(r)}\left(V_{j i t}\right)-\lambda_{j}\left(V_{j i t}\right)\right|^{2}}, \\
\operatorname{RMSE}_{F} & =\frac{1}{R} \sum_{r=1}^{R} \sqrt{\frac{1}{T} \sum_{t=1}^{T} \sum_{j=1}^{2}\left|\widehat{F}_{j t}-F_{j t}\right|^{2}} .
\end{aligned}
$$

\begin{tabular}{|c|c|c|c|c|c|c|c|c|c|}
\hline & \multirow[b]{2}{*}{$N / T$} & \multicolumn{4}{|c|}{ Example 6.1} & \multicolumn{4}{|c|}{ Example 6.2} \\
\hline & & 20 & 40 & 60 & 80 & 20 & 40 & 60 & 80 \\
\hline \multirow[t]{4}{*}{$\mathrm{RMSE}_{\widehat{\beta}}$} & 20 & 0.1943 & 0.1464 & 0.1206 & 0.1079 & 0.1321 & 0.1011 & 0.0832 & 0.0795 \\
\hline & 40 & 0.1357 & 0.1037 & 0.0841 & 0.0746 & 0.0964 & 0.0740 & 0.0664 & 0.0626 \\
\hline & 60 & 0.1108 & 0.0807 & 0.0688 & 0.0620 & 0.0833 & 0.0678 & 0.0571 & 0.0558 \\
\hline & 80 & 0.0984 & 0.0725 & 0.0616 & 0.0537 & 0.0771 & 0.0620 & 0.0532 & 0.0505 \\
\hline \multirow[t]{4}{*}{$\mathrm{RMSE}_{\lambda}$} & 20 & 0.2851 & 0.2728 & 0.2610 & 0.2718 & 0.2316 & 0.2077 & 0.1986 & 0.1977 \\
\hline & 40 & 0.2115 & 0.1987 & 0.1846 & 0.1903 & 0.1643 & 0.1487 & 0.1432 & 0.1396 \\
\hline & 60 & 0.1748 & 0.1573 & 0.1501 & 0.1463 & 0.1368 & 0.1191 & 0.1135 & 0.1144 \\
\hline & 80 & 0.1507 & 0.1350 & 0.1304 & 0.1281 & 0.1195 & 0.1020 & 0.1008 & 0.0973 \\
\hline \multirow[t]{4}{*}{$\mathrm{RMSE}_{F}$} & 20 & 0.3915 & 0.3875 & 0.3892 & 0.3891 & 0.4050 & 0.3978 & 0.3936 & 0.3960 \\
\hline & 40 & 0.2765 & 0.2776 & 0.2776 & 0.2805 & 0.2792 & 0.2781 & 0.2823 & 0.2812 \\
\hline & 60 & 0.2251 & 0.2287 & 0.2301 & 0.2303 & 0.2306 & 0.2294 & 0.2291 & 0.2322 \\
\hline & 80 & 0.1976 & 0.1987 & 0.1981 & 0.2000 & 0.2002 & 0.1985 & 0.2008 & 0.2019 \\
\hline
\end{tabular}

Table 5: Simulation results for RMSEs 

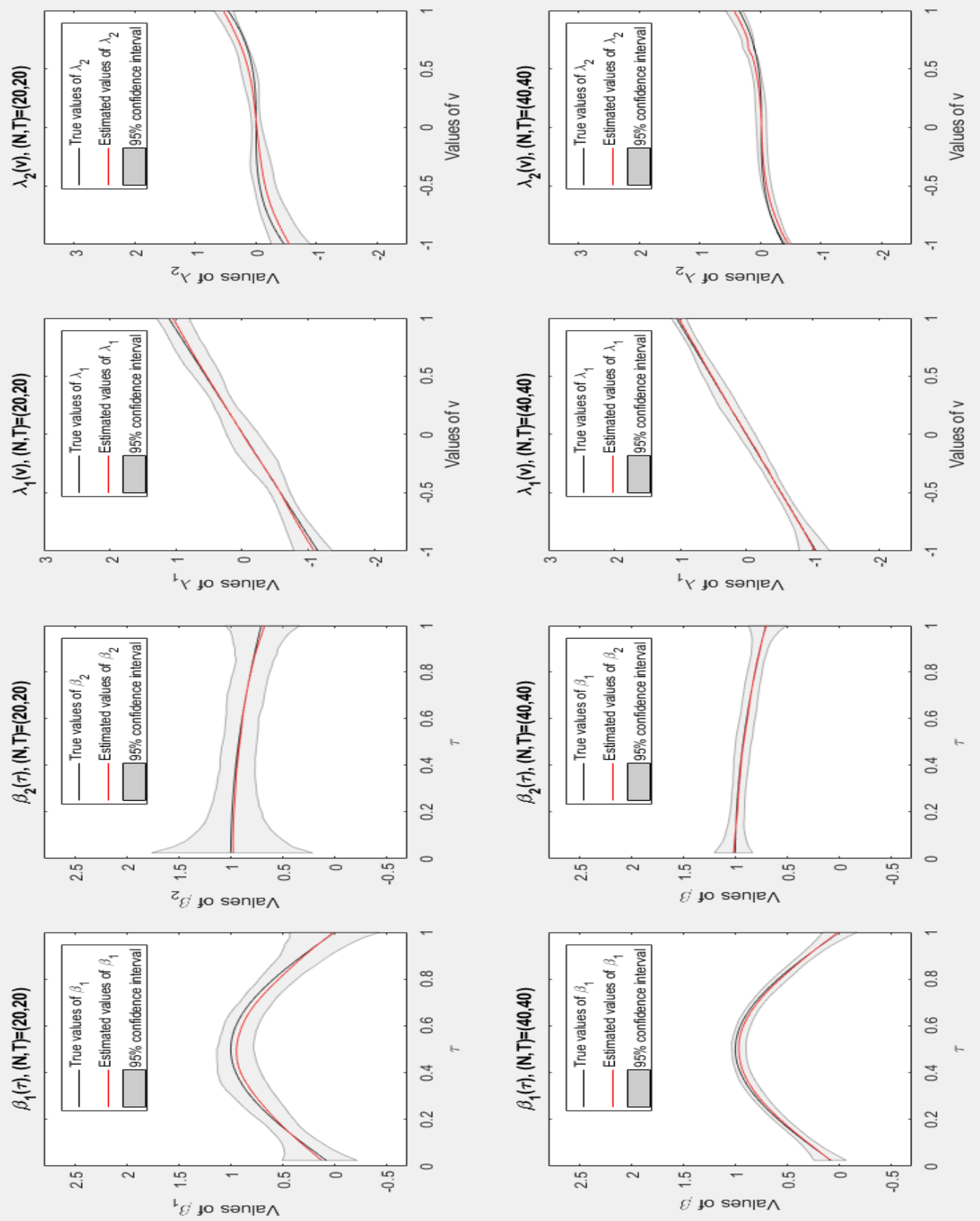

Figure 5: PMI estimates and confidence intervals for Example 6.1 

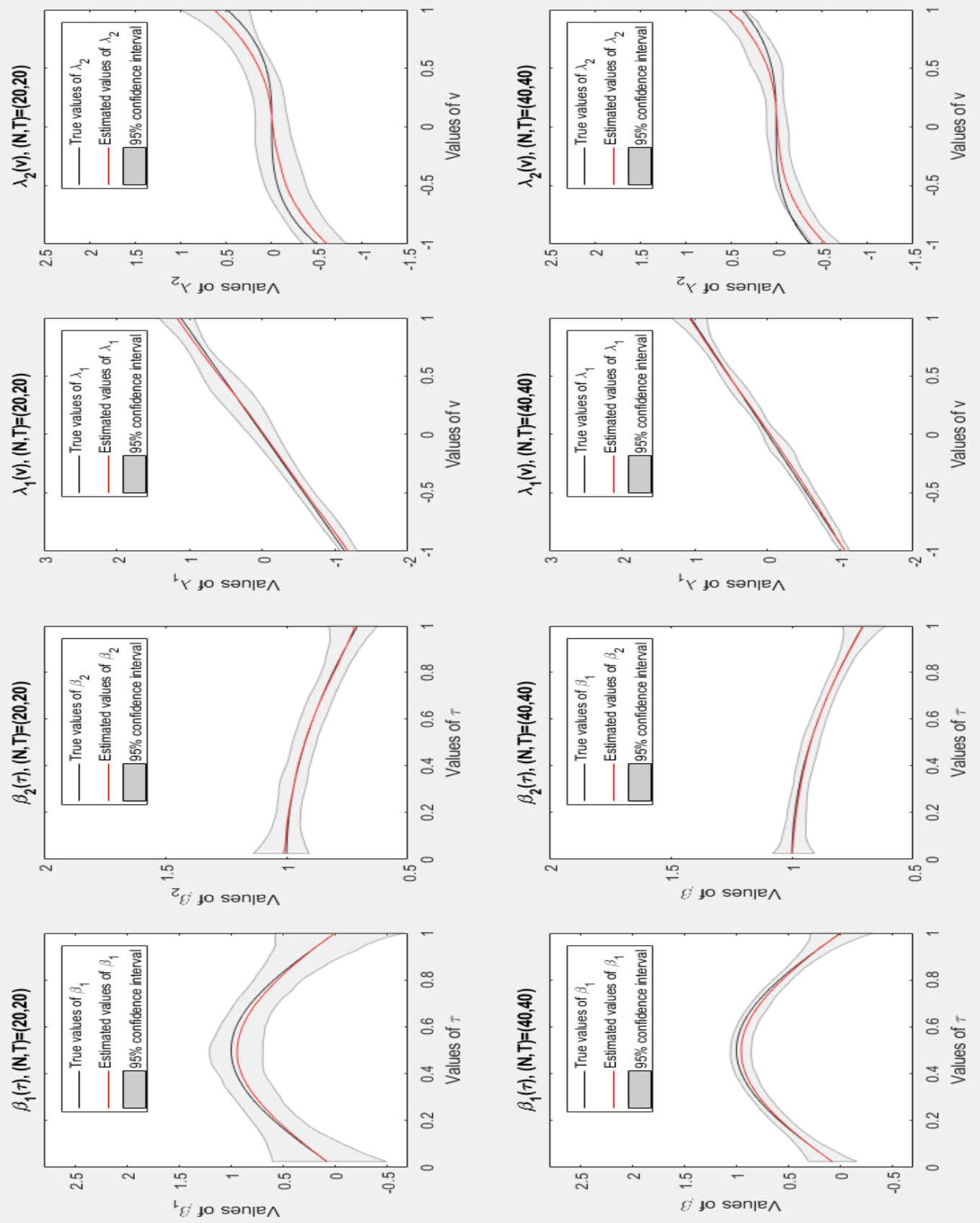

Figure 6: PMI estimates and confidence intervals for Example 6.2

The RMSEs are provided in Table 5. As can be seen from Table 5 , the RMSEs of $\widehat{\boldsymbol{\beta}}(\tau)$ decrease significantly as $(N, T)$ increases from $(20,20)$ to $(80,80)$ in each simulation example. This finding is consistent with Theorem 3.1, which implies $\widehat{\boldsymbol{\beta}}(\tau)$ has root- $N T h$ consistency. In addition, the simulation results from Example 6.2 show the convergence of $\widehat{\boldsymbol{\beta}}(\tau)$ when the correlation between the explanatory variables and loadings is present. It verifies our arguments 
that the consistency of the proposed PMI method is not impaired by the dependency between $\mathbf{x}_{i t}$ and $\mathbf{V}_{i t}$. The RMSEs for the loading and factor estimates decrease as $N$ increases. An increase in $T$ does not eliminate the estimation bias for the factor estimate, which is consistent with our arguments in Theorem 3.4.

For the cases with $(N, T)=(20,20)$ and $(N, T)=(40,40)$, we calculate the simulated $95 \%$ confidence intervals for the estimates. The moving blocks bootstrap (MBB) method proposed by Gonçalves (2011) is employed in our study.

The estimated nonparametric functions and confidence intervals are reported in Figures 5 and 6 . As can be seen from Figures 5 and 6 , the $95 \%$ confidence intervals in each example can converge as $(N, T)$ increases from $(20,20)$ to $(40,40)$. This is also consistent with the simulated RMSEs in Table 5 and our theoretical results in Section 2.

\section{Conclusion}

In this paper, we have proposed a nonparametric panel data model with both time-varying slope coefficients and partially observed additive factor structure. In this flexible model setting, the economic relationship between different variables is allowed to vary over time and the effects of unobservable common factors can be captured by nonparametric loading functions. For this model, we have developed the so-called PMI method that combines the conventional MI approach with the profile local linear method. With this method, we have been able to estimate the unknown functions $\boldsymbol{\beta}(\tau), \boldsymbol{\lambda}(\mathbf{v})$ and parameters $\mathbf{F}_{t}$ simultaneously.

We have then established the asymptotically distributional properties of the proposed PMI estimators for the regression coefficient functions, the loading functions and factors. Finite sample performance of the proposed PMI estimation method is assessed by one simulation study and two empirical examples. In an application in the US financial market, we reveal that excess returns can be explained by the following security characteristics: size, value, momentum and volatility in nonlinear forms. Our findings also suggest that the investor sentiment effect is affecting the US stock returns and their relationship is time-varying from 2000 to 2018 . In addition, we estimate the income elasticity of health care expenditure by the PMI method. Our estimation results show that the income elasticity is below 1 in OECD countries and therefore the health care service or products are necessary goods.

\section{References}

Ang, A., Hodrick, R. J., Xing, Y., and Zhang, X. (2006). The cross-section of volatility and expected returns. The Journal of Finance, 61(1):259-299.

Bai, J. (2009). Panel data models with interactive fixed effects. Econometrica, 77(4):1229-1279.

Baker, M. and Wurgler, J. (2006). Investor sentiment and the cross-section of stock returns. The Journal of Finance, 61(4):1645-1680. 
Baker, M., Wurgler, J., and Yuan, Y. (2012). Global, local, and contagious investor sentiment. Journal of Financial Economics, 104(2):272-287.

Baltagi, B. H. and Moscone, F. (2010). Health care expenditure and income in the oecd reconsidered: Evidence from panel data. Economic Modelling, 27(4):804-811.

Bosq, D. (2012). Nonparametric Statistics for Stochastic Processes: Estimation and Prediction. Lecture Notes in Statistics. Springer New York.

Cai, Z. (2007). Trending time-varying coefficient time series models with serially correlated errors. Journal of Econometrics, 136(1):163-188.

Chen, J., Gao, J., and Li, D. (2012a). A new diagnostic test for cross-section uncorrelatedness in nonparametric panel data models. Econometric Theory, 28(5):1144-1163.

Chen, J., Gao, J., and Li, D. (2012b). Semiparametric trending panel data models with cross-sectional dependence. Journal of Econometrics, 171(1):71-85.

Connor, G., Hagmann, M., and Linton, O. (2012). Efficient semiparametric estimation of the fama-french model and extensions. Econometrica, 80(2):713-754.

Dong, C., Gao, J., and Peng, B. (2015). Semiparametric single-index panel data models with cross-sectional dependence. Journal of Econometrics, 188(1):301-312.

Fama, E. F. and French, K. R. (1992). The cross-section of expected stock returns. Journal of Finance, 47(2):427465 .

Fama, E. F. and French, K. R. (1993). Common risk factors in the returns on stocks and bonds. Journal of Financial Economics, 33(1):3-56.

Fan, J. and Gijbels, I. (1996). Local Polynomial Modelling and Its Applications. Taylor \& Francis, London.

Fan, J., Liao, Y., and Wang, W. (2016). Projected principal component analysis in factor models. Annals of Statistics, 44(1):219.

Fan, J. and Yao, Q. (2003). Nonlinear Time Series: Nonparametric and Parametric Methods. Springer Series in Statistics. Springer, New York.

Gao, J., Lu, Z., and Tjøstheim, D. (2006). Estimation in semiparametric spatial regression. The Annals of Statistics, 34(3):1395-1435.

Gonçalves, S. (2011). The moving blocks bootstrap for panel linear regression models with individual fixed effects. Econometric Theory, 27(5):1048-1082.

Hauck, K. and Zhang, X. (2016). Heterogeneity in the effect of common shocks on healthcare expenditure growth. Health Economics, 25(9):1090-1103.

Im, K. S., Pesaran, M. H., and Shin, Y. (2003). Testing for unit roots in heterogeneous panels. Journal of Econometrics, 115(1):53-74.

Jones, A. M. and Wildman, J. (2008). Health, income and relative deprivation: Evidence from the bhps. Journal of Health Economics, 27(2):308-324.

Lee, J. and Robinson, P. M. (2016). Series estimation under cross-sectional dependence. Journal of Econometrics, 190(1):1-17. 
Li, D., Chen, J., and Gao, J. (2011). Non-parametric time-varying coefficient panel data models with fixed effects. Econometrics Journal, 14(3):387-408.

Li, Q. and Racine, J. S. (2007). Nonparametric Econometrics: Theory and Practice. Princeton University Press, New Jersey.

Ma, S., Linton, O. B., and Gao, J. (2017). Estimation in semiparametric quantile factor models. Working paper at https://arxiv.org/pdf/1708.09507.pdf.

Mammen, E., Linton, O., and Nielsen, J. (1999). The existence and asymptotic properties of a backfitting projection algorithm under weak conditions. Annals of Statistics, 27(5):1443-1490.

Newey, W. K. (1994). Kernel estimation of partial means and a general variance estimator. Econometric Theory, $10(2): 1-21$.

Newey, W. K. and Powell, J. L. (2003). Instrumental variable estimation of nonparametric models. Econometrica, $71(5): 1565-1578$.

Nielsen, J. P. and Linton, O. (1998). An optimization interpretation of integration and back-fitting estimators for separable nonparametric models. Journal of the Royal Statistical Society: Series B (Statistical Methodology), $60(1): 217-222$.

Pesaran, M. H. (2006). Estimation and inference in large heterogeneous panels with a multifactor error structure. Econometrica, 74(4):967-1012.

Robinson, P. M. (2012). Nonparametric trending regression with cross-sectional dependence. Journal of Econometrics, 169(1):4-14.

Santos, T. and Veronesi, P. (2006). Labor income and predictable stock returns. The Review of Financial Studies, $19(1): 1-44$.

Shao, Q.-M. and Yu, H. (1996). Weak convergence for weighted empirical processes of dependent sequences. The Annals of Probability, 24(4):2098-2127.

Silvapulle, P., Smyth, R., Zhang, X., and Fenech, J.-P. (2017). Nonparametric panel data model for crude oil and stock market prices in net oil importing countries. Energy Economics, 67:255-267.

Stambaugh, R. F., Yu, J., and Yuan, Y. (2012). The short of it: Investor sentiment and anomalies. Journal of Financial Economics, 104(2):288-302.

Su, L., Shi, Z., and Phillips, P. C. (2016). Identifying latent structures in panel data. Econometrica, 84(6):22152264.

Su, L. and Wang, X. (2017). On time--varying factor models: Estimation and testing. Journal of Econometrics, 198(1):84-101.

Tjøstheim, D. and Auestad, B. H. (1994). Nonparametric identification of nonlinear time series: projections. Journal of the American Statistical Association, 89(428):1398-1409. 


\section{Appendix A Proofs of the main results}

Before we present the proofs, we first introduce the following new notation:

$$
\begin{aligned}
& \mathbf{s}_{0}(\tau)=[1,0]\left(\mathbf{M}_{b}^{\top}(\tau) \mathbf{W}_{b}(\tau) \mathbf{M}_{b}(\tau)\right)^{-1} \mathbf{M}_{b}^{\top}(\tau) \mathbf{W}_{b}(\tau), \\
& \widetilde{\gamma}_{i \cdot}=\left(\gamma_{i 1}, \ldots, \gamma_{i T}\right)^{\top}, \quad \varepsilon_{i \cdot}=\left(\varepsilon_{i 1}, \ldots, \varepsilon_{i T}\right)^{\top} \\
& R_{i t}=\mathbf{s}_{0}\left(\tau_{t}\right) \mathbf{y}_{i} \cdot-\sum_{l=1}^{p} \mathbf{s}_{0}\left(\tau_{t}\right) \mathbf{x}_{i .}^{(l)} \beta_{l}\left(\tau_{t}\right) \\
& \mathcal{H}_{N, t}^{\gamma}(\mathbf{v})=\widehat{E}\left[\gamma_{t}\left(\mathbf{V}_{i t}\right) \mid \mathbf{V}_{i t}=\mathbf{v}\right], \quad \mathcal{H}_{N, t}^{\varepsilon}(\mathbf{v})=\widehat{E}\left[\varepsilon_{i t} \mid \mathbf{V}_{i t}=\mathbf{v}\right], \quad \mathcal{H}_{N, t}^{R}(\mathbf{v})=\widehat{E}\left[R_{i t} \mid \mathbf{V}_{i t}=\mathbf{v}\right], \\
& \widehat{\mathcal{P}}_{j t}^{\gamma}\left(v_{j}\right)=\frac{1}{N} \sum_{i=1}^{N} \mathcal{H}_{N, t}^{\gamma}\left(\mathbf{V}_{i t}^{(-j)}\left(v_{j}\right)\right) W^{(-j)}\left(\mathbf{V}_{i t}^{(-j)}\right) w_{j}\left(v_{j}\right), \\
& \widehat{\mathcal{P}}_{j t}^{\varepsilon}\left(v_{j}\right)=\frac{1}{N} \sum_{i=1}^{N} \mathcal{H}_{N, t}^{\varepsilon}\left(\mathbf{V}_{i t}^{(-j)}\left(v_{j}\right)\right) W^{(-j)}\left(\mathbf{V}_{i t}^{(-j)}\right) w_{j}\left(v_{j}\right), \\
& \widehat{\mathcal{P}}_{j t}^{R}\left(v_{j}\right)=\frac{1}{N} \sum_{i=1}^{N} \mathcal{H}_{N, t}^{R}\left(\mathbf{V}_{i t}^{(-j)}\left(v_{j}\right)\right) W^{(-j)}\left(\mathbf{V}_{i t}^{(-j)}\right) w_{j}\left(v_{j}\right), \\
& \widehat{\mathbf{P}}_{j t}^{V}\left(v_{j}\right)=\left(\widehat{\mathcal{P}}_{j t}^{(1)}\left(v_{j}\right), \ldots, \widehat{\mathcal{P}}_{j t}^{(p)}\left(v_{j}\right)\right)^{\top}, \quad \mathbf{P}_{j t}^{V}\left(v_{j}\right)=\left(\mathcal{P}_{j t}^{(1)}\left(v_{j}\right), \ldots, \mathcal{P}_{j t}^{(p)}\left(v_{j}\right)\right)^{\top} \\
& \mathcal{N}(\tau)=\left(\mathcal{X}_{1}\left(\frac{\tau_{1}-\tau}{h_{0}}\right)^{2}, \ldots, \mathcal{X}_{T}\left(\frac{\tau_{T}-\tau}{h_{0}}\right)^{2}\right)^{\top} .
\end{aligned}
$$

In addition, for notational simplicity, let $m_{X, i}^{(0)}(\tau)=m_{Y, i}(\tau)$ and $\widehat{m}_{X, i}^{(0)}(\tau)=\widehat{m}_{Y, i}(\tau)$.

For the proof of the main theorems, we introduce the following lemmas. Lemma A.1 derives several useful rates of convergence. The asymptotic distributions under $\alpha$-mixing conditions are established in Lemma A.2. The uniform consistency of the marginal integration projection matrix is provided by Lemma A.3. The leading terms in the marginal integration projection are given in Lemma A.4. Also for notational simplicity, we define $K_{t, m}(\tau)=K\left(\frac{\tau_{t}-\tau}{h_{0}}\right)\left(\frac{\tau_{t}-\tau}{h_{0}}\right)^{m}$ and $K_{j i t, m}\left(v_{j}\right)=K\left(\frac{V_{j i t}-v_{j}}{h_{j}}\right)\left(\frac{V_{j i t}-v_{j}}{h_{j}}\right)^{m}$, for $m=0,1,2,3$.

Lemma A.1. Let Assumptions 1-3 hold. We have

(1) $\sum_{i=1}^{N} \sum_{t=1}^{T} K_{t, m}(\tau)\left(\mathbf{x}_{i t}^{*} \mathbf{x}_{i t}^{* \top}-E\left[\mathbf{x}_{i t}^{*} \mathbf{x}_{i t}^{* \top}\right]\right)=O_{P}\left(\sqrt{N T h_{0}}\right)$,

(2) $\sum_{i=1}^{N} \sum_{t=1}^{T} \sum_{s=1}^{T} K_{s, m}\left(\tau_{t}\right) \mathcal{P}_{j t}^{(l)}\left(v_{j}\right) x_{i s}^{(l) *} \varepsilon_{i s}=O_{P}\left(\sqrt{N T^{3} h_{0}^{2}}\right)$,

(3) $\sup _{\tau \in(0,1)}\left\|\frac{1}{N T h_{0}} \sum_{i=1}^{N} \sum_{t=1}^{T} K_{t, m}(\tau)\left(\mathbf{x}_{i t}^{*} \mathbf{x}_{i t}^{* \top}-E\left[\mathbf{x}_{i t}^{*} \mathbf{x}_{i t}^{* \top}\right]\right)\right\|=o_{P}(1)$,

(4) $\sup _{\tau \in(0,1)}\left\|\widehat{m}_{X, i}^{(l)}(\tau)-m_{X, i}^{(l)}(\tau)\right\|=o_{P}(1)$,

for $m=0,1,2,3$ and $l=1,2, \ldots, p$.

Lemma A.2. Let Assumptions 1-4 hold. We have

(1) $\frac{1}{\sqrt{N T h_{0}}} \sum_{i=1}^{N} \sum_{t=1}^{T} K_{t, 0}(\tau) \widetilde{\mathbf{x}}_{i t}^{*} \varepsilon_{i t} \stackrel{D}{\longrightarrow} \mathcal{N}\left(\mathbf{0}_{p}, \boldsymbol{\Sigma}_{\beta}^{0}\right)$,

(2) $\frac{1}{\sqrt{N T h_{j}}} \sum_{i=1}^{N} \sum_{t=1}^{T} K\left(\frac{V_{j i t}-v_{j}}{h_{j}}\right) \xi_{j i t}\left(v_{j}\right) \varepsilon_{i t} \stackrel{D}{\longrightarrow} \mathcal{N}\left(0, \Sigma_{j}^{V}\left(v_{j}\right)\right)$,

where $\xi_{j i t}\left(v_{j}\right)=p_{V}^{-1}\left(\mathbf{V}_{i t}^{(-j)}\left(v_{j}\right)\right) p_{V}^{(-j)}\left(\mathbf{V}_{i t}^{(-j)}\right) W^{(-j)}\left(\mathbf{V}_{i t}^{(-j)}\right) w_{j}\left(v_{j}\right), \phi_{j i t}=p_{V, j}\left(V_{j i t}\right) \xi_{j i t}\left(V_{j i t}\right), \widetilde{\mathbf{x}}_{i t}^{*}=\breve{x}_{i t}^{(l)}+$ $\breve{x}_{i t}^{(l) c}\left(1-\sum_{j=1}^{J} \phi_{j i t}\right)$, and $\boldsymbol{\Sigma}_{\beta}^{0}$ and $\Sigma_{j}^{V}\left(v_{j}\right)$ are defined in Assumption 4 . 
Lemma A.3. Let Assumptions 1-3 hold. We have the following uniform convergence:

$$
\sup _{v_{j} \in\left[\underline{v}_{j}, \bar{v}_{j}\right]}\left|\widehat{\mathcal{P}}_{j t}^{(l)}\left(v_{j}\right)-\mathcal{P}_{j t}^{(l)}\left(v_{j}\right)\right|=o_{P}(1) .
$$

Lemma A.4. Let Assumptions 1-3 hold. For $v_{j} \in\left[\underline{v}_{j}, \bar{v}_{j}\right]$, we have $\widehat{\mathcal{P}}_{j t}^{(0)}\left(v_{j}\right)-\mathcal{P}_{j t}^{(0)}\left(v_{j}\right)-\boldsymbol{\beta}^{\top}\left(\tau_{t}\right)\left(\widehat{\mathbf{P}}_{j t}^{V}\left(v_{j}\right)-\mathbf{P}_{j t}^{V}\left(v_{j}\right)\right)=T_{j t}^{a}\left(v_{j}\right)+T_{j t}^{b}\left(v_{j}\right)+T_{j t}^{c}\left(v_{j}\right)+o_{P}\left(\sum_{j=1}^{J} h_{j}^{2}\right)+o_{P}\left(\frac{1}{\sqrt{N h_{j}}}\right)$, where

$$
\begin{aligned}
& T_{j t}^{a}\left(v_{j}\right)=\frac{1}{N} \sum_{i=1}^{N} \sum_{k \neq j}\left(\gamma_{k t}\left(V_{k i t}\right) W^{(-j)}\left(\mathbf{V}_{i t}^{(-j)}\right)-E\left[\gamma_{k t}\left(V_{k i t}\right) W^{(-j)}\left(\mathbf{V}_{i t}^{(-j)}\right)\right]\right) \\
& T_{j t}^{b}\left(v_{j}\right)=b_{j t}^{*}\left(v_{j}\right) h_{j}^{2}+\sum_{k \neq j} \frac{\mu_{2, k} h_{k}^{2}}{2} E\left[\gamma_{k t}^{\prime \prime}\left(V_{k i t}\right) W^{(-j)}\left(\mathbf{V}_{i t}^{(-j)}\right)\right] \\
& T_{j t}^{c}\left(v_{j}\right)=\frac{1}{N h_{j}} \sum_{n=1}^{N} K\left(\frac{V_{j n t}-v_{j}}{h_{j}}\right) p_{V}^{-1}\left(\mathbf{V}_{n t}^{(-j)}\left(v_{j}\right)\right) p_{V}^{(-j)}\left(\mathbf{V}_{n t}^{(-j)}\right) W^{(-j)}\left(\mathbf{V}_{n t}^{(-j)}\right) \varepsilon_{n t}
\end{aligned}
$$

with $b_{j t}^{*}\left(v_{j}\right)=\frac{\mu_{2, j}}{2} \gamma_{j t}^{\prime \prime}\left(v_{j}\right)$. In addition, for $T_{j t}^{c}\left(v_{j}\right)$, we have $\sum_{i=1}^{N} \sum_{t=1}^{T} T_{j t}^{c}\left(V_{j i t}\right)=O_{P}(\sqrt{N T})$.

Lemma A.5. Let Assumptions 1-3 hold. We have

(1) $\sum_{i=1}^{N} \sum_{t=1}^{T} \mathcal{G}_{i t}=O_{P}\left(N T \sum_{k=1}^{J} h_{k}^{2}\right)$,

(2) $\sum_{i=1}^{N} \sum_{t=1}^{T} K_{t, 0}(\tau) \mathbf{x}_{i t}^{*} \mathcal{G}_{i t}=O_{P}\left(N T h_{0} \sum_{k=1}^{J} h_{k}^{2}\right)$,

(3) $\sum_{i=1}^{N} \sum_{t=1}^{T} K_{t, 0}(\tau) \mathbf{x}_{i t}^{*} \mathcal{R}_{i t}=O_{P}\left(N T h_{0}\left(b^{2}+\sum_{k=1}^{J} h_{k}^{2}\right)\right)$,

where $\mathcal{G}_{i t}=\sum_{j=1}^{J}\left(\gamma_{j t}\left(V_{j i t}\right)-\widehat{\mathcal{P}}_{j t}^{\gamma}\left(V_{j i t}\right)\right)$ and $\mathcal{R}_{i t}=R_{i t}-\widehat{\mathcal{P}}_{j t}^{R}\left(V_{j i t}\right)$.

Lemma A.6. Let Assumptions 1-3 hold. We have

$$
\sum_{i=1}^{N} \sum_{t=1}^{T} K_{t, 0}(\tau) \mathbf{x}_{i t}^{*} \mathcal{E}_{i t}=\sum_{i=1}^{N} \sum_{t=1}^{T} K_{t, 0}(\tau) \widetilde{\mathbf{x}}_{i t}^{*} \varepsilon_{i t}+o_{P}\left(\sqrt{N T h_{0}}\right) .
$$

The proofs of the main lemmas are provided in Appendix B. With Lemmas A.1-A.4, we can prove the main theorems.

\section{Proof of Theorem 3.1:}

Recall that $y_{i t}^{*}=\widetilde{y}_{i t}-\sum_{j=1}^{J} \mathcal{P}_{j t}^{(0)}\left(V_{j i t}\right), x_{i t}^{(l) *}=\widetilde{x}_{i t}^{(l)}-\sum_{j=1}^{J} \mathcal{P}_{j t}^{(l)}\left(V_{j i t}\right)$ and $\mathbf{x}_{i t}^{*}=\left(x_{i t}^{(1) *}, \ldots, x_{i t}^{(p) *}\right)^{\top}$. In addition, we defined the notation: $\widehat{y}_{i t}^{*}=\widehat{\widetilde{y}}_{i t}-\sum_{j=1}^{J} \widehat{\mathcal{P}}_{j t}^{(0)}\left(V_{j i t}\right), \widehat{x}_{i t}^{(l) *}=\widehat{\widetilde{x}}_{i t}^{(l)}-\sum_{j=1}^{J} \widehat{\mathcal{P}}_{j t}^{(l)}\left(V_{j i t}\right)$ and $\widehat{\mathbf{x}}_{i t}^{*}=\left(\widehat{x}_{i t}^{(1) *}, \ldots, \widehat{x}_{i t}^{(p) *}\right)^{\top}$. For $\mathcal{H}_{N, t}^{(0)}(\mathbf{v})$ and $\widehat{\mathcal{P}}_{j t}^{(0)}\left(v_{j}\right)$, we have

$$
\mathcal{H}_{N, t}^{(0)}(\mathbf{v})=\sum_{l=1}^{p} \beta_{l}\left(\tau_{t}\right) \mathcal{H}_{N, t}^{(l)}(\mathbf{v})+\mathcal{H}_{N, t}^{\gamma}(\mathbf{v})+\mathcal{H}_{N, t}^{\varepsilon}(\mathbf{v})-\mathcal{H}_{N, t}^{R}(\mathbf{v})
$$

and $\widehat{\mathcal{P}}_{j t}^{(0)}\left(v_{j}\right)=\sum_{l=1}^{p} \beta_{l}\left(\tau_{t}\right) \widehat{\mathcal{P}}_{j t}^{(l)}\left(v_{j}\right)+\widehat{\mathcal{P}}_{j t}^{\gamma}\left(v_{j}\right)+\widehat{\mathcal{P}}_{j t}^{\varepsilon}\left(v_{j}\right)-\widehat{\mathcal{P}}_{j t}^{R}\left(v_{j}\right)$.

For $\widehat{y}_{i t}^{*}$, by local linear approximation

$$
\widehat{y}_{i t}^{*}=\sum_{l=1}^{p} \beta_{l}\left(\tau_{t}\right)\left(x_{i t}^{(l)}-\sum_{j=1}^{J} \widehat{\mathcal{P}}_{j t}^{(l)}\left(V_{j i t}\right)\right)+\sum_{j=1}^{J}\left(\gamma_{j t}\left(V_{j i t}\right)-\widehat{\mathcal{P}}_{j t}^{\gamma}\left(V_{j i t}\right)\right)+\varepsilon_{i t}-\sum_{j=1}^{J} \widehat{\mathcal{P}}_{j t}^{\varepsilon}\left(V_{j i t}\right)
$$




$$
\begin{aligned}
& =\widehat{\mathbf{x}}_{i t}^{* \top} \boldsymbol{\beta}\left(\tau_{t}\right)+\mathcal{G}_{i t}+\mathcal{E}_{i t}-\mathcal{R}_{i t} \\
& =\widehat{\mathbf{x}}_{i t}^{* \top}\left(\boldsymbol{\beta}(\tau)+\boldsymbol{\beta}(\tau)^{\prime}\left(\frac{\tau_{t}-\tau}{h_{0}}\right)+\frac{h_{0}^{2}}{2} \boldsymbol{\beta}^{\prime \prime}(\tau)\left(\frac{\tau_{t}-\tau}{h_{0}}\right)^{2}+o\left(h_{0}^{2}\right)\right)+\mathcal{G}_{i t}+\mathcal{E}_{i t}-\mathcal{R}_{i t},
\end{aligned}
$$

where $\mathcal{G}_{i t}=\sum_{j=1}^{J}\left(\gamma_{j t}\left(V_{j i t}\right)-\widehat{\mathcal{P}}_{j t}^{\gamma}\left(V_{j i t}\right)\right), \mathcal{E}_{i t}=\varepsilon_{i t}-\sum_{j=1}^{J} \widehat{\mathcal{P}}_{j t}^{\varepsilon}\left(V_{j i t}\right)$ and $\mathcal{R}_{i t}=R_{i t}-\sum_{j=1}^{J} \widehat{\mathcal{P}}_{j t}^{R}\left(V_{j i t}\right)$.

Let $\mathcal{G}_{t}=\left(\mathcal{G}_{1 t}, \cdots, \mathcal{G}_{N t}\right)^{\top}, \mathcal{G}=\left(\mathcal{G}_{1}^{\top}, \ldots, \mathcal{G}_{T}^{\top}\right)^{\top}, \mathcal{E}_{t}=\left(\mathcal{E}_{1 t}, \cdots, \mathcal{E}_{N t}\right)^{\top}, \mathcal{E}=\left(\mathcal{E}_{1}^{\top}, \ldots, \mathcal{E}_{T}^{\top}\right)^{\top}, \mathcal{R}_{t}=$ $\left(\mathcal{R}_{1 t}, \cdots, \mathcal{R}_{N t}\right)^{\top}$ and $\mathcal{R}=\left(\mathcal{R}_{1}^{\top}, \ldots, \mathcal{R}_{T}^{\top}\right)^{\top}$. With this notation, we have

$$
\begin{aligned}
& \widehat{\boldsymbol{\beta}}(\tau)-\boldsymbol{\beta}(\tau)=\left[\mathbf{I}_{p}, \mathbf{0}_{p}\right]\left(\mathcal{X}^{\top}(\tau) \mathbf{W}(\tau) \mathcal{X}(\tau)\right)^{-1} \mathcal{X}^{\top}(\tau) \mathbf{W}(\tau) \mathcal{N}(\tau)\left(\frac{h^{2}}{2} \beta^{\prime \prime}(\tau)+o\left(h_{0}^{2}\right)\right) \\
& +\left[\mathbf{I}_{p}, \mathbf{0}_{p}\right]\left(\mathcal{X}^{\top}(\tau) \mathbf{W}(\tau) \mathcal{X}(\tau)\right)^{-1} \mathcal{X}^{\top}(\tau) \mathbf{W}(\tau) \mathcal{G}+\left[\mathbf{I}_{p}, \mathbf{0}_{p}\right]\left(\mathcal{X}^{\top}(\tau) \mathbf{W}(\tau) \mathcal{X}(\tau)\right)^{-1} \mathcal{X}^{\top}(\tau) \mathbf{W}(\tau) \mathcal{E} \\
& +\left[\mathbf{I}_{p}, \mathbf{0}_{p}\right]\left(\mathcal{X}^{\top}(\tau) \mathbf{W}(\tau) \mathcal{X}(\tau)\right)^{-1} \mathcal{X}^{\top}(\tau) \mathbf{W}(\tau) \mathcal{R} \\
& =\mathbf{A}_{N T, 1}(\tau)+\cdots+\mathbf{A}_{N T, 4}(\tau) .
\end{aligned}
$$

For the inverse part, define

$$
\begin{aligned}
& \widehat{\boldsymbol{\Omega}}_{A}(\tau)=\frac{1}{N T h} \mathcal{X}^{\top}(\tau) \mathbf{W}(\tau) \mathcal{X}(\tau) \\
& =\left(\begin{array}{cc}
\frac{1}{N T h_{0}} \sum_{i=1}^{N} \sum_{t=1}^{T} K_{t, 0}(\tau) \widehat{\mathbf{x}}_{i t}^{*} \widehat{\mathbf{x}}_{i t}^{* \top} & \frac{1}{N T h_{0}} \sum_{i=1}^{N} \sum_{t=1}^{T} K_{t, 1}(\tau) \widehat{\mathbf{x}}_{i t}^{*} \widehat{\mathbf{x}}_{i t}^{* \top} \\
\frac{1}{N T h_{0}} \sum_{i=1}^{N} \sum_{t=1}^{T} K_{t, 1}(\tau) \widehat{\mathbf{x}}_{i t}^{*} \widehat{\mathbf{x}}_{i t}^{* \top} & \frac{1}{N T h_{0}} \sum_{i=1}^{N} \sum_{t=1}^{T} K_{t, 2}(\tau) \widehat{\mathbf{x}}_{i t}^{*} \widehat{\mathbf{x}}_{i t}^{* \top}
\end{array}\right) \\
& =\left(\begin{array}{cc}
\widehat{\boldsymbol{\Omega}}_{11}^{A}(\tau) & \widehat{\boldsymbol{\Omega}}_{12}^{A}(\tau) \\
\widehat{\boldsymbol{\Omega}}_{12}^{A}(\tau) & \widehat{\boldsymbol{\Omega}}_{22}^{A}(\tau)
\end{array}\right),
\end{aligned}
$$

where $K_{t, m}(\tau)=K\left(\frac{\tau_{t}-\tau}{h_{0}}\right)\left(\frac{\tau_{t}-\tau}{h_{0}}\right)^{m}$ for $m=1,2,3$.

Let $\boldsymbol{\Delta}_{i t}^{X}=\widehat{\mathbf{x}}_{i t}^{*}-\mathbf{x}_{i t}^{*}$. For $\widehat{\Omega}_{11}^{A}(\tau)$, it is clear to see that

$$
\begin{aligned}
& \widehat{\boldsymbol{\Omega}}_{11}^{A}(\tau)=\frac{1}{N T h_{0}} \sum_{i=1}^{N} \sum_{t=1}^{T} K_{t, 0}(\tau) \mathbf{x}_{i t}^{*} \mathbf{x}_{i t}^{* \top}+\frac{1}{N T h_{0}} \sum_{i=1}^{N} \sum_{t=1}^{T} K_{t, 0}(\tau) \boldsymbol{\Delta}_{i t}^{X} \mathbf{x}_{i t}^{* \top} \\
& +\frac{1}{N T h_{0}} \sum_{i=1}^{N} \sum_{t=1}^{T} K_{t, 0}(\tau) \mathbf{x}_{i t}^{*} \boldsymbol{\Delta}_{i t}^{X \top}+\frac{1}{N T h_{0}} \sum_{i=1}^{N} \sum_{t=1}^{T} K_{t, 0}(\tau) \boldsymbol{\Delta}_{i t}^{X} \boldsymbol{\Delta}_{i t}^{X \top} \\
& =\widehat{\boldsymbol{\Omega}}_{11,1}^{A}(\tau)+\cdots+\widehat{\boldsymbol{\Omega}}_{11,4}^{A}(\tau) .
\end{aligned}
$$

By Lemmas A.1 and A.3, it is clear to see that

$$
\begin{aligned}
& \sum_{i=1}^{N} \sum_{t=1}^{T} K_{t, 0}(\tau)\left\|\boldsymbol{\Delta}_{i t}^{X}\right\|^{2}=\sum_{i=1}^{N} \sum_{t=1}^{T} \sum_{l=1}^{p} K_{t, 0}(\tau)\left|\widehat{m}_{X, i}^{(l)}\left(\tau_{t}\right)-m_{X, i}^{(l)}\left(\tau_{t}\right)\right|^{2} \\
& +\sum_{i=1}^{N} \sum_{t=1}^{T} \sum_{l=1}^{p} K_{t, 0}(\tau)\left|\sum_{j=1}^{J}\left(\widehat{\mathcal{P}}_{j t}^{(l)}\left(V_{j i t}\right)-\mathcal{P}_{j t}^{(l)}\left(V_{j i t}\right)\right)\right|^{2}+\text { cross products }=o_{P}\left(N T h_{0}\right) .
\end{aligned}
$$

By (A.2) and Cauchy-Schwarz inequality, we have

$$
\left\|\widehat{\boldsymbol{\Omega}}_{11,2}^{A}(\tau)\right\| \leq \frac{1}{N T h_{0}}\left(\sum_{i=1}^{N} \sum_{t=1}^{T} K_{t, 0}(\tau)\left\|\mathbf{x}_{i t}^{* \top}\right\|^{2}\right)^{\frac{1}{2}}\left(\sum_{i=1}^{N} \sum_{t=1}^{T} K_{t, 0}(\tau)\left\|\boldsymbol{\Delta}_{i t}^{X}\right\|^{2}\right)^{\frac{1}{2}}=o_{P}(1) .
$$

In a similar way to (A.3), we have

$$
\left\|\widehat{\boldsymbol{\Omega}}_{11,3}^{A}(\tau)\right\|=o_{P}(1), \quad\left\|\widehat{\boldsymbol{\Omega}}_{11,4}^{A}(\tau)\right\|=o_{P}(1) .
$$


By (A.3) and (A.4), for $m=2,3,4$,

$$
\left\|\sum_{m=2}^{4} \widehat{\Omega}_{11, m}^{A}(\tau)\right\|=o_{P}(1) .
$$

By the properties of $\alpha$-mixing processes and Lemma A.1,

$$
\widehat{\boldsymbol{\Omega}}_{11,1}^{A}(\tau)-\mathbf{\Sigma}_{A}=\frac{1}{N T h} \sum_{i=1}^{N} \sum_{t=1}^{T} K_{t, 0}(\tau)\left(\mathbf{x}_{i t}^{*} \mathbf{x}_{i t}^{* \top}-E\left[\mathbf{x}_{i t}^{*} \mathbf{x}_{i t}^{* \top}\right]\right)+O\left(\frac{1}{T h_{0}}\right)=o_{P}(1)
$$

where $\boldsymbol{\Sigma}_{A}$ is defined in Assumption 4 and the first equality holds by the property of Riemann integral. It follows from (A.5) and (A.6) that $\widehat{\boldsymbol{\Omega}}_{11}^{A}(\tau) \stackrel{P}{\longrightarrow} \boldsymbol{\Sigma}_{A}$.

Analogously, we can show that $\widehat{\boldsymbol{\Omega}}_{12}^{A}(\tau) \stackrel{P}{\longrightarrow} \mathbf{0}_{P}$ and $\widehat{\boldsymbol{\Omega}}_{22}^{A}(\tau) \stackrel{P}{\longrightarrow} \mu_{2} \boldsymbol{\Sigma}_{A}$, where $\mu_{2}=\int K(u) u^{2} d u$.

It is followed by

$$
\widehat{\boldsymbol{\Omega}}^{A}(\tau) \stackrel{P}{\longrightarrow} \boldsymbol{\Omega}_{A}
$$

where $\boldsymbol{\Omega}_{A}=\operatorname{diag}\left(1, \mu_{2}\right) \otimes \boldsymbol{\Sigma}_{A}$.

Similarly to (A.7), we have

$$
\frac{1}{N T h_{0}} \mathcal{X}^{\top}(\tau) \mathbf{W}(\tau) \mathcal{N}(\tau) \stackrel{P}{\longrightarrow}\left(\mu_{2}, 0\right)^{\top} \otimes \boldsymbol{\Sigma}_{A}
$$

By (A.7) and (A.8), we have $\mathbf{A}_{N T, 1}(\tau)=\frac{\mu_{2}}{2} \boldsymbol{\beta}^{\prime \prime}(\tau) h_{0}^{2}+o_{P}\left(h_{0}^{2}\right)$.

Then we proceed with $\mathbf{A}_{N T, 2}(\tau)$. For $\mathbf{A}_{N T, 2}(\tau)$, note that

$$
\frac{1}{N T h_{0}} \sum_{i=1}^{N} \sum_{t=1}^{T} K_{t, 0}(\tau) \widehat{\mathbf{x}}_{i t}^{*} \mathcal{G}_{i t}=\frac{1}{N T h_{0}} \sum_{i=1}^{N} \sum_{t=1}^{T} K_{t, 0}(\tau) \mathbf{x}_{i t}^{*} \mathcal{G}_{i t}+\frac{1}{N T h_{0}} \sum_{i=1}^{N} \sum_{t=1}^{T} K_{t, 0}(\tau) \boldsymbol{\Delta}_{i t}^{X} \mathcal{G}_{i t} .
$$

By Lemma A.5, we have

$$
\frac{1}{N T h_{0}} \sum_{i=1}^{N} \sum_{t=1}^{T} K_{t, 0}(\tau) \mathbf{x}_{i t}^{*} \mathcal{G}_{i t}=O_{P}\left(\sum_{k=1}^{J} h_{k}^{2}\right) .
$$

By Lemmas A.1, A.3 and A.5, and Cauchy-Schwarz inequality, we have

$$
\frac{1}{N T h_{0}} \sum_{i=1}^{N} \sum_{t=1}^{T} K_{t, 0}(\tau) \boldsymbol{\Delta}_{i t}^{X} \mathcal{G}_{i t}=o_{P}\left(\sum_{k=1}^{J} h_{k}^{2}\right)
$$

Therefore, for $\mathbf{A}_{N T, 2}(\tau)$, we have $\mathbf{A}_{N T, 2}(\tau)=O_{P}\left(\sum_{k=1}^{J} h_{k}^{2}\right)$.

For $\mathbf{A}_{N T, 3}(\tau)$, it is clear to see that

$$
\begin{aligned}
& \frac{1}{N T h_{0}} \sum_{i=1}^{N} \sum_{t=1}^{T} K_{t, 0}(\tau) \widehat{\mathbf{x}}_{i t}^{*} \mathcal{E}_{i t}=\frac{1}{N T h_{0}} \sum_{i=1}^{N} \sum_{t=1}^{T} K_{t, 0}(\tau) \mathbf{x}_{i t}^{*} \mathcal{E}_{i t}+\frac{1}{N T h_{0}} \sum_{i=1}^{N} \sum_{t=1}^{T} K_{t, 0}(\tau) \boldsymbol{\Delta}_{i t}^{X} \mathcal{E}_{i t} \\
& =\frac{1}{N T h_{0}} \sum_{i=1}^{N} \sum_{t=1}^{T} K_{t, 0}(\tau) \mathbf{x}_{i t}^{*} \mathcal{E}_{i t}+o_{P}\left(\frac{1}{\sqrt{N T h_{0}}}\right),
\end{aligned}
$$

where the last equality holds by Lemmas A.1 and A.3, and Cauchy-Schwarz inequality.

Recall that $\widetilde{\mathbf{x}}_{i t}^{*}=\breve{x}_{i t}^{(l)}+\breve{x}_{i t}^{(l) c}\left(1-\sum_{j=1}^{J} \phi_{j i t}\right)$, where

$$
\phi_{j i t}=W^{(-j)}\left(\mathbf{V}_{i t}^{(-j)}\right) w_{j}\left(V_{j i t}\right) p_{V}^{-1}\left(\mathbf{V}_{i t}\right) p_{V}^{(-j)}\left(\mathbf{V}_{i t}^{(-j)}\right) p_{V, j}\left(V_{j i t}\right) .
$$


By (A.6), Lemmas A.2 and A.6, we obtain

$$
\begin{aligned}
& \sqrt{N T h_{0}} \mathbf{A}_{N T, 3}=\frac{1}{\sqrt{N T h_{0}}} \boldsymbol{\Sigma}_{A}^{-1} \sum_{i=1}^{N} \sum_{t=1}^{T} K_{t, 0}(\tau) \mathbf{x}_{i t}^{*} \mathcal{E}_{i t} \\
& =\frac{1}{\sqrt{N T h_{0}}} \boldsymbol{\Sigma}_{A}^{-1} \sum_{i=1}^{N} \sum_{t=1}^{T} K_{t, 0}(\tau) \widetilde{\mathbf{x}}_{i t}^{*} \varepsilon_{i t}+o_{P}(1) \stackrel{D}{\longrightarrow} \mathcal{N}\left(\mathbf{0}_{p}, \boldsymbol{\Sigma}_{A}^{-1} \boldsymbol{\Sigma}_{\beta}^{0} \boldsymbol{\Sigma}_{A}^{-1}\right),
\end{aligned}
$$

where $\boldsymbol{\Sigma}_{A}$ and $\boldsymbol{\Sigma}_{\beta}^{0}$ are defined in Assumption 4 .

For $\mathbf{A}_{N T, 4}$, by Lemmas A.1, A.3 and A.5, and Cauchy-Schwarz inequality, it is implied

$$
\begin{aligned}
& \frac{1}{N T h_{0}} \sum_{i=1}^{N} \sum_{t=1}^{T} K_{t, 0}(\tau) \widehat{\mathbf{x}}_{i t}^{*} \mathcal{R}_{i t}=\frac{1}{N T h_{0}} \sum_{i=1}^{N} \sum_{t=1}^{T} K_{t, 0}(\tau) \mathbf{x}_{i t}^{*} \mathcal{R}_{i t}+\frac{1}{N T h_{0}} \sum_{i=1}^{N} \sum_{t=1}^{T} K_{t, 0}(\tau) \Delta_{i t}^{X} \mathcal{R}_{i t} \\
& =O_{P}\left(b^{2}+\sum_{j=1}^{J} h_{j}^{2}\right) .
\end{aligned}
$$

It finishes the proof of Theorem 3.1.

\section{Proof of Theorem 3.3}

For notational simplicity, we first prove Theorem 3.3. For $v_{j} \in\left[\bar{v}_{j}, \underline{v}_{j}\right]$, we have

$$
\begin{aligned}
& \widehat{\gamma}_{j t}\left(v_{j}\right)=\widehat{\mathcal{P}}_{j t}\left(v_{j}, \widehat{\boldsymbol{\beta}}\left(\tau_{t}\right)\right)-\frac{1}{N} \sum_{i=1}^{N} \widehat{\mathcal{P}}_{j t}\left(V_{j i t}, \widehat{\boldsymbol{\beta}}\left(\tau_{t}\right)\right) \\
& =\left(\widehat{\mathcal{P}}_{j t}\left(v_{j}, \widehat{\boldsymbol{\beta}}\left(\tau_{t}\right)\right)-\widehat{\mathcal{P}}_{j t}\left(v_{j}, \boldsymbol{\beta}\left(\tau_{t}\right)\right)-\frac{1}{N} \sum_{i=1}^{N}\left(\widehat{\mathcal{P}}_{j t}\left(V_{j i t}, \widehat{\boldsymbol{\beta}}\left(\tau_{t}\right)\right)-\widehat{\mathcal{P}}_{j t}\left(V_{j i t}, \boldsymbol{\beta}\left(\tau_{t}\right)\right)\right)\right) \\
& +\left(\widehat{\mathcal{P}}_{j t}\left(v_{j}, \boldsymbol{\beta}\left(\tau_{t}\right)\right)-\mathcal{P}_{j t}\left(v_{j}, \boldsymbol{\beta}\left(\tau_{t}\right)\right)-\frac{1}{N} \sum_{i=1}^{N}\left(\widehat{\mathcal{P}}_{j t}\left(V_{j i t}, \boldsymbol{\beta}\left(\tau_{t}\right)\right)-\mathcal{P}_{j t}\left(V_{j i t}, \boldsymbol{\beta}\left(\tau_{t}\right)\right)\right)\right) \\
& +\left(\mathcal{P}_{j t}\left(v_{j}, \boldsymbol{\beta}\left(\tau_{t}\right)\right)-\frac{1}{N} \sum_{i=1}^{N} \mathcal{P}_{j t}\left(V_{j i t}, \boldsymbol{\beta}\left(\tau_{t}\right)\right)\right)=B_{N, 1 t}\left(v_{j}\right)+B_{N, 2 t}\left(v_{j}\right)+B_{N, 3 t}\left(v_{j}\right) .
\end{aligned}
$$

where $\mathcal{P}_{j t}\left(v_{j}, \boldsymbol{\beta}\left(\tau_{t}\right)\right)=\mathcal{P}_{j t}^{(0)}\left(v_{j}\right)-\sum_{l=1}^{p} \beta_{l}\left(\tau_{t}\right) \mathcal{P}_{j t}^{(l)}\left(v_{j}\right)$. It is sufficient to show that

$$
\sqrt{N h_{j}} B_{N, 1 t}\left(v_{j}\right)=o_{P}(1), \quad \sqrt{N h_{j}}\left(B_{N, 3 t}\left(v_{j}\right)-\gamma_{j t}\left(v_{j}\right)\right)=o_{P}(1),
$$

and

$$
\sqrt{N h_{j}}\left(B_{N, 2 t}\left(v_{j}\right)\right) \stackrel{D}{\longrightarrow} \mathcal{N}\left(0, \Sigma_{j t}^{V}\left(v_{j}\right)\right) .
$$

For $B_{N, 1 t}\left(v_{j}\right)$

$$
\begin{aligned}
& \widehat{\mathcal{P}}_{j t}\left(v_{j}, \widehat{\boldsymbol{\beta}}\left(\tau_{t}\right)\right)-\widehat{\mathcal{P}}_{j t}\left(v_{j}, \boldsymbol{\beta}\left(\tau_{t}\right)\right)=-\left(\widehat{\boldsymbol{\beta}}\left(\tau_{t}\right)-\boldsymbol{\beta}\left(\tau_{t}\right)\right)^{\top} \widehat{\mathbf{P}}_{j t}^{V}\left(v_{j}\right) \\
& =O_{P}\left(\frac{1}{\sqrt{N T h_{0}}}\right)+O_{P}\left(h_{0}^{2}\right) .
\end{aligned}
$$

Therefore, we have $B_{N, 1 t}\left(v_{j}\right)=O_{P}\left(\frac{1}{\sqrt{N T h_{0}}}\right)+O_{P}\left(h_{0}^{2}\right)$. Note that by the condition $N T h_{0}^{5}=O(1)$ in Assumption 3, we have $B_{N, 1 t}\left(v_{j}\right)=o_{P}\left(\frac{1}{\sqrt{N h_{j}}}\right)$.

For $B_{N, 2 t}\left(v_{j}\right)$, it is clear to see that

$$
\widehat{\mathcal{P}}_{j t}\left(v_{j}, \boldsymbol{\beta}\left(\tau_{t}\right)\right)-\mathcal{P}_{j t}\left(v_{j}, \boldsymbol{\beta}\left(\tau_{t}\right)\right)=\widehat{\mathcal{P}}_{j t}^{(0)}\left(v_{j}\right)-\mathcal{P}_{j t}^{(0)}\left(v_{j}\right)-\sum_{l=1}^{p} \beta_{l}\left(\tau_{t}\right)\left(\widehat{\mathcal{P}}_{j t}^{(l)}\left(v_{j}\right)-\mathcal{P}_{j t}^{(l)}\left(v_{j}\right)\right)
$$




$$
=T_{j t}^{a}+T_{j t}^{b}\left(v_{j}\right)+T_{j t}^{c}\left(v_{j}\right)+o_{P}\left(\frac{1}{\sqrt{N h_{j}}}\right),
$$

where the last equality follows directly from Lemma A.4, and $T_{j t}^{a}\left(v_{j}\right), T_{j t}^{b}\left(v_{j}\right), T_{j t}^{c}\left(v_{j}\right)$ are defined in Lemma A.4.

Therefore, by the weak cross-sectional dependence and the law of large numbers, we have

$$
\begin{aligned}
& B_{N, 2 t}\left(v_{j}\right)=T_{j t}^{b}\left(v_{j}\right)+T_{j t}^{c}\left(v_{j}\right)-\frac{1}{N} \sum_{i=1}^{N}\left(T_{j t}^{b}\left(V_{j i t}\right)+T_{j t}^{c}\left(V_{j i t}\right)\right)+o_{P}\left(\frac{1}{\sqrt{N h_{j}}}\right) \\
& =T_{j t}^{c}\left(v_{j}\right)+o_{P}\left(\frac{1}{\sqrt{N h_{j}}}\right) .
\end{aligned}
$$

By Assumption 5, we further have that $\sqrt{N h_{j}} T_{j t}^{c}\left(v_{j}\right) \stackrel{D}{\longrightarrow} \mathcal{N}\left(0, \Sigma_{j t}^{V}\left(v_{j}\right)\right)$, which implies that (A.10) holds.

Recall that $\mathcal{P}_{j t}^{(l)}\left(v_{j}\right)=E\left[\mathcal{H}_{t}^{(l)}\left(\mathbf{V}_{i t}^{(-j)}\left(v_{j}\right)\right) W^{(-j)}\left(\mathbf{V}_{i t}^{(-j)}\right)\right] w_{j}\left(v_{j}\right), \mathcal{H}_{t}^{(0)}(\mathbf{v})=E\left[y_{i t} \mid \mathbf{V}_{i t}=\mathbf{v}\right]$ and $\mathcal{H}_{t}^{(s)}(\mathbf{v})=E\left[x_{i t}^{(s)} \mid \mathbf{V}_{i t}=\mathbf{v}\right]$. For $B_{N, 3 t}\left(v_{j}\right)$, note that

$$
\mathcal{P}_{j t}\left(v_{j}, \boldsymbol{\beta}\left(\tau_{t}\right)\right)=\mathcal{P}_{j t}^{(0)}\left(v_{j}\right)-\sum_{l=1}^{p} \beta_{l}\left(\tau_{t}\right) \mathcal{P}_{j t}^{(l)}\left(v_{j}\right)=\gamma_{j t}\left(v_{j}\right)+\sum_{k \neq j} E\left[\gamma_{k t}\left(V_{k i t}\right) W^{(-j)}\left(\mathbf{V}_{i t}^{(-j)}\right)\right] .
$$

It is followed by

$$
\begin{aligned}
& B_{N, 3 t}\left(v_{j}\right)=\mathcal{P}_{j t}\left(v_{j}, \boldsymbol{\beta}\left(\tau_{t}\right)\right)-\frac{1}{N} \sum_{i=1}^{N} \mathcal{P}_{j t}\left(V_{j i}, \boldsymbol{\beta}\left(\tau_{t}\right)\right) \\
& =\gamma_{j t}\left(v_{j}\right)-\frac{1}{N} \sum_{i=1}^{N} \gamma_{j t}\left(V_{j i t}\right)=\gamma_{j t}\left(v_{j}\right)+O_{P}\left(\frac{1}{\sqrt{N}}\right),
\end{aligned}
$$

where the last equality holds by the weak cross-sectional dependence.

Therefore, we have shown that Theorem 3.3 holds.

\section{Proof of Theorem 3.2:}

By the identification conditions in Assumption 1, we have

$$
\widehat{\lambda}_{j}\left(v_{j}\right)-\lambda_{j}\left(v_{j}\right)=\frac{\frac{1}{T} \sum_{t=1}^{T} \widehat{\gamma}_{j t}\left(v_{j}\right)}{\sqrt{\int\left(\frac{1}{T} \sum_{t=1}^{T} \widehat{\gamma}_{j t}\left(v_{j}\right)\right)^{2} d P_{V, j}(v)}}-\frac{\frac{1}{T} \sum_{t=1}^{T} \gamma_{j t}\left(v_{j}\right)}{\sqrt{\int\left(\frac{1}{T} \sum_{t=1}^{T} \gamma_{j t}\left(v_{j}\right)\right)^{2} d P_{V, j}(v)}} .
$$

It is sufficient to show that for $v_{j} \in\left[\underline{v}_{j}, \bar{v}_{j}\right]$,

$$
\sqrt{N T h_{j}}\left(\frac{1}{T} \sum_{t=1}^{T} \bar{F}_{j}^{-1}\left(\widehat{\gamma}_{j t}\left(v_{j}\right)-\gamma_{j t}\left(v_{j}\right)\right)-c_{j}\left(v_{j}\right) h_{j}^{2}\right) \stackrel{D}{\longrightarrow} \mathcal{N}\left(0, \Sigma_{j}^{V}\left(v_{j}\right)\right)
$$

where $c_{j}\left(v_{j}\right)=\frac{\mu_{2}}{2}\left(\lambda_{j}^{\prime \prime}\left(v_{j}\right)-E\left[\lambda_{j}^{\prime \prime}\left(V_{j i}\right)\right]\right)\left(1+o_{P}(1)\right)$.

By (A.9), we have

$$
\frac{1}{T} \sum_{t=1}^{T} \bar{F}_{j}^{-1}\left(\widehat{\gamma}_{j t}\left(v_{j}\right)-\gamma_{j t}\left(v_{j}\right)\right)=\frac{1}{T} \sum_{t=1}^{T} \bar{F}_{j}^{-1}\left(B_{N, 1 t}\left(v_{j}\right)+B_{N, 2 t}\left(v_{j}\right)+B_{N, 3 t}\left(v_{j}\right)-\gamma_{j t}\left(v_{j}\right)\right),
$$


where $B_{N, 1 t}\left(v_{j}\right), B_{N, 2 t}\left(v_{j}\right)$ and $B_{N, 3 t}\left(v_{j}\right)$ are defined in (A.9).

Recall that $\widehat{\mathbf{P}}_{j t}^{V}\left(v_{j}\right)=\left(\widehat{\mathcal{P}}_{j t}^{(1)}\left(v_{j}\right), \ldots, \widehat{\mathcal{P}}_{j t}^{(p)}\left(v_{j}\right)\right)^{\top}$ and $\mathbf{P}_{j t}^{V}\left(v_{j}\right)=\left(\mathcal{P}_{j t}^{(1)}\left(v_{j}\right), \ldots, \mathcal{P}_{j t}^{(p)}\left(v_{j}\right)\right)^{\top}$.

For $\frac{1}{T} \sum_{t=1}^{T} B_{N, 1 t}\left(v_{j}\right)$, with the condition $h_{0}^{2}+\sum_{k \neq j} h_{k}^{2}=o\left(h_{j}^{2}\right)$, we have

$$
\begin{aligned}
& \frac{1}{T} \sum_{t=1}^{T}\left(\widehat{\mathcal{P}}_{j t}\left(v_{j}, \widehat{\boldsymbol{\beta}}\left(\tau_{t}\right)\right)-\widehat{\mathcal{P}}_{j t}\left(v_{j}, \boldsymbol{\beta}\left(\tau_{t}\right)\right)\right)=-\frac{1}{T} \sum_{t=1}^{T}\left(\widehat{\boldsymbol{\beta}}\left(\tau_{t}\right)-\boldsymbol{\beta}\left(\tau_{t}\right)\right)^{\top} \widehat{\mathbf{P}}_{j t}^{V}\left(v_{j}\right) \\
& =-\frac{1}{T} \sum_{t=1}^{T} \mathbf{A}_{N T, 3}\left(\tau_{t}\right)^{\top} \mathbf{P}_{j t}^{V}\left(v_{j}\right)+O_{P}\left(h_{0}^{2}\right)+o_{P}\left(\frac{1}{\sqrt{N T h_{j}}}\right) \\
& =O_{P}\left(\frac{1}{\sqrt{N T}}\right)+O_{P}\left(h_{0}^{2}\right)+o_{P}\left(\frac{1}{\sqrt{N T h_{j}}}\right)=o_{P}\left(h_{j}^{2}\right)+o_{P}\left(\frac{1}{\sqrt{N T h_{j}}}\right),
\end{aligned}
$$

where $\mathbf{A}_{N T, 3}(\tau)$ is defined in (A.1) and the second equality holds by Lemmas A.1 and A.3.

For $\frac{1}{T} \sum_{t=1}^{T} B_{N, 2 t}\left(v_{j}\right)$, by Lemma A.4, we obtain

$$
\begin{aligned}
& \frac{1}{T} \bar{F}_{j}^{-1} \sum_{t=1}^{T} B_{N, 2 t}\left(v_{j}\right)=\frac{1}{T} \bar{F}_{j}^{-1} \sum_{t=1}^{T}\left(T_{j t}^{b}\left(v_{j}\right)+T_{j t}^{c}\left(v_{j}\right)\right)-\frac{1}{N T} \bar{F}_{j}^{-1} \sum_{i=1}^{N} \sum_{t=1}^{T}\left(T_{j t}^{b}\left(V_{j i t}\right)+T_{j t}^{c}\left(V_{j i t}\right)\right) \\
& +o_{P}\left(\frac{1}{\sqrt{N T h_{j}}}\right)=c_{j}\left(v_{j}\right) h_{j}^{2}+\frac{1}{T} \bar{F}_{j}^{-1} \sum_{t=1}^{T} T_{j t}^{c}\left(v_{j}\right)+o_{P}\left(\frac{1}{\sqrt{N T h_{j}}}\right)
\end{aligned}
$$

By Lemma A.2, we have

$$
\sqrt{N T h_{j}}\left(\frac{1}{T} \bar{F}_{j}^{-1} \sum_{t=1}^{T} B_{N, 2 t}\left(v_{j}\right)-c_{j}\left(v_{j}\right) h_{j}^{2}\right) \stackrel{D}{\longrightarrow} \mathcal{N}\left(0, \Sigma_{j}^{V}\left(v_{j}\right)\right),
$$

where $\Sigma_{j}^{V}\left(v_{j}\right)$ is defined in Theorem 3.2.

For $\frac{1}{T} \sum_{t=1}^{T} B_{N, 3 t}\left(v_{j}\right)$,

$$
\begin{aligned}
& \frac{1}{T} \bar{F}_{j}^{-1} \sum_{t=1}^{T} B_{N, 3 t}\left(v_{j}\right)=\frac{1}{T} \bar{F}_{j}^{-1} \sum_{t=1}^{T} \mathcal{P}_{j t}\left(v_{j}, \boldsymbol{\beta}\left(\tau_{t}\right)\right)-\frac{1}{N T} \bar{F}_{j}^{-1} \sum_{t=1}^{T} \sum_{i=1}^{N} \mathcal{P}_{j t}\left(V_{j i t}, \boldsymbol{\beta}\left(\tau_{t}\right)\right) \\
& =\lambda_{j}\left(v_{j}\right)-\frac{1}{N T} \bar{F}_{j}^{-1} \sum_{t=1}^{T} \sum_{i=1}^{N} \lambda_{j}\left(V_{j i t}\right) F_{j t}=\lambda_{j}\left(v_{j}\right)+O_{P}\left(\frac{1}{\sqrt{N T}}\right) .
\end{aligned}
$$

\section{Proof of Theorem 3.4}

For each $j$ and any given $t$, we have

$$
\widehat{F}_{j t}-F_{j t}=\left(\sum_{i=1}^{N} \widehat{\lambda}_{j}^{2}\left(V_{j i t}\right)\right)^{-1} \sum_{i=1}^{N} \widehat{\lambda}_{j}\left(V_{j i t}\right)\left(\widehat{\gamma}_{j t}\left(V_{j i t}\right)-\widehat{\lambda}_{j}\left(V_{j i t}\right) F_{j t}\right) .
$$

By Theorem 3.2 and the law of large numbers, it is clear to see that

$$
\frac{1}{N} \sum_{i=1}^{N} \widehat{\lambda}_{j}^{2}\left(V_{j i t}\right) \stackrel{P}{\longrightarrow} E\left[\lambda_{j}^{2}\left(V_{j i t}\right)\right]
$$

In addition, we have

$$
\frac{1}{N} \sum_{i=1}^{N} \widehat{\lambda}_{j}\left(V_{j i t}\right)\left(\widehat{\gamma}_{j t}\left(V_{j i t}\right)-\widehat{\lambda}_{j}\left(V_{j i t}\right) F_{j t}\right)=\frac{1}{N} \sum_{i=1}^{N} \widehat{\lambda}_{j}\left(V_{j i t}\right)\left(\widehat{\gamma}_{j t}\left(V_{j i t}\right)-\gamma_{j t}\left(V_{j i t}\right)\right)
$$




$$
+\frac{1}{N} \sum_{i=1}^{N} \widehat{\lambda}_{j}\left(V_{j i t}\right)\left(\widehat{\lambda}_{j}\left(V_{j i t}\right)-\lambda_{j}\left(V_{j i t}\right)\right) F_{j t}
$$

For its second term, by Lemma A.3,

$$
\begin{aligned}
& \frac{1}{N} \sum_{i=1}^{N} \widehat{\lambda}_{j}\left(V_{j i t}\right)\left(\widehat{\lambda}_{j}\left(V_{j i t}\right)-\lambda_{j}\left(V_{j i t}\right)\right)=\frac{1}{N} \sum_{i=1}^{N} \lambda_{j}\left(V_{j i t}\right)\left(\widehat{\lambda}_{j}\left(V_{j i t}\right)-\lambda_{j}\left(V_{j i t}\right)\right)\left(1+o_{P}(1)\right) \\
& =\frac{1}{N} \sum_{i=1}^{N} \lambda_{j}\left(V_{j i t}\right)\left(\frac{1}{T} \sum_{s=1}^{T} \bar{F}_{j}^{-1}\left(B_{N, 1 s}\left(V_{j i t}\right)+B_{N, 2 s}\left(V_{j i t}\right)+B_{N, 3 s}\left(V_{j i t}\right)-\gamma_{j t}\left(V_{j i t}\right)\right)\right)\left(1+o_{P}(1)\right),
\end{aligned}
$$

where $B_{N, 1 t}\left(v_{j}\right), B_{N, 2 t}\left(v_{j}\right), B_{N, 3 t}\left(v_{j}\right)$ are defined in (A.9).

By Lemma A.4, analogously to (A.11), (A.12) and (A.13), we have

$$
\frac{1}{N} \sum_{i=1}^{N} \widehat{\lambda}_{j}\left(V_{j i t}\right)\left(\widehat{\lambda}_{j}\left(V_{j i t}\right)-\lambda_{j}\left(V_{j i t}\right)\right)=O_{P}\left(h_{0}^{2}\right)+O_{P}\left(h_{j}^{2}\right)+o_{P}\left(\frac{1}{\sqrt{N}}\right) .
$$

Therefore, by Assumption 3, we have

$$
\begin{aligned}
& \frac{1}{N} \sum_{i=1}^{N} \widehat{\lambda}_{j}\left(V_{j i t}\right)\left(\widehat{\gamma}_{j t}\left(V_{j i t}\right)-\widehat{\lambda}_{j}\left(V_{j i t}\right) F_{j t}\right)=\frac{1}{N} \sum_{i=1}^{N} \lambda_{j}\left(V_{j i t}\right)\left(\widehat{\gamma}_{j t}\left(V_{j i t}\right)-\gamma_{j t}\left(V_{j i t}\right)\right)+o_{P}\left(\frac{1}{\sqrt{N}}\right) \\
& =\frac{1}{N} \sum_{i=1}^{N} \lambda_{j}\left(V_{j i t}\right)\left(B_{N, 1 t}\left(V_{j i t}\right)+B_{N, 2 t}\left(V_{j i t}\right)+B_{N, 3 t}\left(V_{j i t}\right)-\gamma_{j t}\left(V_{j i t}\right)\right)+o_{P}\left(\frac{1}{\sqrt{N}}\right) \\
& =\frac{1}{N} \sum_{i=1}^{N} \lambda_{j}\left(V_{j i t}\right) T_{j t}^{c}\left(V_{j i t}\right)+O_{P}\left(h_{0}^{2}\right)+O_{P}\left(h_{j}^{2}\right)+o_{P}\left(\frac{1}{\sqrt{N}}\right) \\
& =\frac{1}{N} \sum_{i=1}^{N} \lambda_{j}\left(V_{j i t}\right) T_{j t}^{c}\left(V_{j i t}\right)+o_{P}\left(\frac{1}{\sqrt{N}}\right),
\end{aligned}
$$

where $T_{j t}^{c}\left(v_{j}\right)$ is defined in Lemma A.4.

By Assumption 5, we have

$$
\begin{aligned}
& \sqrt{N}\left(\widehat{F}_{j t}-F_{j t}\right)=\sqrt{N}\left(\sum_{i=1}^{N} \widehat{\lambda}_{j}^{2}\left(V_{j i t}\right)\right)^{-1} \sum_{i=1}^{N} \lambda_{j}\left(V_{j i t}\right) T_{j t}^{c}\left(V_{j i t}\right)+o_{P}\left(\frac{1}{\sqrt{N}}\right) \\
& \stackrel{D}{\longrightarrow} \mathcal{N}\left(0, \Sigma_{t}^{F}\right) .
\end{aligned}
$$

We have therefore completed the proofs of Theorems 3.1-3.4. 


\title{
Supplementary Document to "Time-Varying Panel Data Model with an Additive Factor Structure"
}

\author{
FEI $\mathrm{LIU}^{\dagger}$, Jiti GAO $\mathrm{G}^{\ddagger}$ AND YANRONG YANG $\sharp$ \\ Nankai University, China ${ }^{\dagger}$ \\ Monash University, Australia ${ }^{\ddagger}$
}

The Australian National University, Australia $\sharp^{\sharp}$

This supplementary document contains Appendix B that lists lemmas that are used in Appendix A as well as their proofs, and Appendix $\mathrm{C}$ that gives some additional discussion on the proposed model and its estimation method.

\section{Appendix B Proofs of the main lemmas}

In this section, we provide the proofs of Lemmas A.1-A.4 listed in Appendix A, and then give some secondary lemmas and their proofs.

\section{B.1 Proofs of Lemmas A.1-A.4}

\section{Proof of Lemma A.1}

Without loss of generality, we prove the case with $m=1$. Then for other values of $m$, the lemma can be proved analogously.

(1) It is straightforward to see that

$$
E\left[\frac{1}{N T h_{0}} \sum_{i=1}^{N} \sum_{t=1}^{T} K_{t, 0}(\tau)\left(\mathbf{x}_{i t}^{*} \mathbf{x}_{i t}^{* \top}-E\left[\mathbf{x}_{i t}^{*} \mathbf{x}_{i t}^{* \top}\right]\right)\right]=\mathbf{0}_{p} .
$$

For simplicity, we define that $\boldsymbol{\xi}_{i t}^{X}=\mathbf{x}_{i t}^{*} \mathbf{x}_{i t}^{* \top}-E\left[\mathbf{x}_{i t}^{*} \mathbf{x}_{i t}^{* \top}\right]$. By the properties of strictly stationary and $\alpha$-mixing processes, we have that $\boldsymbol{\xi}_{i t}^{X}$ is strictly stationary and the $\alpha$-mixing properties in Assumption 2.(1) can be satisfied.

Then we have

$$
\begin{aligned}
& E\left[\left\|\frac{1}{N T h_{0}} \sum_{i=1}^{N} \sum_{t=1}^{T} K_{t, 0}(\tau) \xi_{i t}^{X}\right\|^{2}\right]=\frac{1}{N^{2} T^{2} h_{0}^{2}} \sum_{l_{1}=1}^{p} \sum_{l_{2}=1}^{p} \sum_{i=1}^{N} \sum_{n=1}^{N} \sum_{t=1}^{T} \sum_{s=1}^{T} K_{t, 0}(\tau) K_{s, 0}(\tau) E\left[\xi_{l_{1} l_{2}, i t}^{X} \xi_{l_{2} l_{1}, n s}^{X}\right] \\
& =\frac{1}{N^{2} T^{2} h_{0}^{2}} \sum_{l_{1}=1}^{p} \sum_{l_{2}=1}^{p} \sum_{i=1}^{N} \sum_{n=1}^{N} \sum_{t=1}^{T} K_{t, 0}^{2}(\tau) E\left[\xi_{l_{1} l_{2}, i t}^{X} \xi_{l_{2} l_{1}, n t}^{X}\right] \\
& +\frac{1}{N^{2} T^{2} h_{0}^{2}} \sum_{l_{1}=1}^{p} \sum_{l_{2}=1}^{p} \sum_{i=1}^{N} \sum_{n=1}^{N} \sum_{t=1}^{T} \sum_{s=t+1}^{T} K_{t, 0}(\tau) K_{s, 0}(\tau) E\left[\xi_{l_{1} l_{2}, i t}^{X} \xi_{l_{2} l_{1}, n s}^{X}\right] \\
& +\frac{1}{N^{2} T^{2} h_{0}^{2}} \sum_{l_{1}=1}^{p} \sum_{l_{2}=1}^{p} \sum_{i=1}^{N} \sum_{n=1}^{N} \sum_{t=1}^{T} \sum_{s=1}^{t-1} K_{t, 0}(\tau) K_{t, 0}(\tau) E\left[\xi_{l_{1} l_{2}, i t}^{X} \xi_{l_{2} l_{1}, n s}^{X}\right] \\
& =\frac{1}{N^{2} T^{2} h_{0}^{2}} \sum_{l_{1}=1}^{p} \sum_{l_{2}=1}^{p} \sum_{i=1}^{N} \sum_{n=1}^{N}\left(D_{i n, 1}^{l_{1} l_{2}}+D_{i n, 2}^{l_{1} l_{2}}+D_{i n, 3}^{l_{1} l_{2}}\right)
\end{aligned}
$$


where $\xi_{l_{1} l_{2}, i t}^{X}$ is the $\left(l_{1}, l_{2}\right)$-th element of $\boldsymbol{\xi}_{i t}^{X}$.

For $D_{i n, 2}^{l_{1} l_{2}}$, it is clear to see that

$$
\begin{aligned}
& \left|D_{i n, 2}^{l_{1} l_{2}}\right|=\sum_{t=1}^{T} \sum_{s=t+1}^{T} K_{t, 0}(\tau) K_{s, 0}(\tau)\left|\operatorname{Cov}\left(\xi_{l_{1} l_{2}, i t}^{X}, \xi_{l_{2} l_{1}, n s}^{X}\right)\right| \\
& =\sum_{t=1}^{T-1} \sum_{s=1}^{T-t} K_{t, 0}(\tau) K_{t+s, 0}(\tau)\left|\operatorname{Cov}\left(\xi_{l_{1} l_{2}, i t}^{X}, \xi_{l_{2} l_{1}, n, t+s}^{X}\right)\right| \\
& \leq C \sum_{t=1}^{T-1} \sum_{s=1}^{T-t} K_{t, 0}(\tau)\left|\operatorname{Cov}\left(\xi_{l_{1} l_{2}, i t}^{X}, \xi_{l_{2} l_{1}, n, t+s}^{X}\right)\right|=C \sum_{t=1}^{T-1} K_{t, 0}(\tau) \sum_{s=1}^{T-t}\left|\operatorname{Cov}\left(\xi_{l_{1} l_{2}, i 1}^{X}, \xi_{l_{2} l_{1}, n, 1+s}^{X}\right)\right| \\
& \leq C c_{\delta} T h_{0} \sum_{s=1}^{T-1} \alpha_{i j}(s)^{\delta /(4+\delta)}\left(E\left[\left|\xi_{l_{1} l_{2}, i 1}^{X}\right|^{2+\delta / 2}\right]\right)^{2 /(4+\delta)}\left(E\left[\left|\xi_{l_{2} l_{1}, n, 1+s}^{X}\right|^{2+\delta / 2}\right]\right)^{2 /(4+\delta)} \\
& \leq C T h_{0} \sum_{s=1}^{T-1} \alpha_{i j}(s)^{\delta /(4+\delta)},
\end{aligned}
$$

where $c_{\delta}=(4+\delta) / \delta \cdot 2^{(4+2 \delta) /(4+\delta)}$ and the second last inequality in (B.2) holds by Davydov's inequality (see pages 19-20 in Bosq (2012) and Dong et al. (2015)).

By Assumption 2 and (B.2), we have

$$
\sum_{l_{1}=1}^{p} \sum_{l_{2}=1}^{p} \sum_{i=1}^{N} \sum_{n=1}^{N}\left|D_{i n, 2}^{l_{1} l_{2}}\right| \leq C N T h_{0} .
$$

Analogously to (B.3), we have

$$
\sum_{l_{1}=1}^{p} \sum_{l_{2}=1}^{p} \sum_{i=1}^{N} \sum_{n=1}^{N}\left|D_{i n, 1}^{l_{1} l_{2}}\right| \leq C N T h_{0} \text { and } \sum_{l_{1}=1}^{p} \sum_{l_{2}=1}^{p} \sum_{i=1}^{N} \sum_{n=1}^{N}\left|D_{i n, 3}^{l_{1} l_{2}}\right| \leq C N T h_{0} .
$$

By (B.3) and (B.4),

$$
E\left[\left\|\frac{1}{N T h_{0}} \sum_{i=1}^{N} \sum_{t=1}^{T} K_{t, 0}(\tau) \boldsymbol{\xi}_{i t}^{X}\right\|^{2}\right]=O_{P}\left(\frac{1}{N T h_{0}}\right),
$$

which implies that Lemma A.1 holds.

(2) By Assumption 2, we can show that

$$
E\left[\sum_{i=1}^{N} \sum_{t=1}^{T} \sum_{s=1}^{T} K_{s, m}\left(\tau_{t}\right) \mathcal{P}_{j t}^{(l)}\left(v_{j}\right) x_{i s}^{(l) *} \varepsilon_{i s}\right]=0 .
$$

For its second moment,

$$
\begin{aligned}
& E\left[\left\|\sum_{i=1}^{N} \sum_{t=1}^{T} \sum_{s=1}^{T} K_{s, m}\left(\tau_{t}\right) \mathcal{P}_{j t}^{(l)}\left(v_{j}\right) x_{i s}^{(l) *} \varepsilon_{i s}\right\|^{2}\right]=\sum_{i=1}^{N} \sum_{n=1}^{N} \sum_{t_{1}=1}^{T} \sum_{t_{2}=1}^{T} \sum_{s_{1}=1}^{T} \sum_{s_{2}=1}^{T} K_{s_{1}, 0}\left(\tau_{t_{1}}\right) K_{s_{2}, 0}\left(\tau_{t_{2}}\right) \\
& \times \mathcal{P}_{j t_{1}}^{(l)}\left(v_{j}\right) \mathcal{P}_{j t_{2}}^{(l)}\left(v_{j}\right) E\left[x_{i s_{1}}^{(l) *} x_{n s_{2}}^{(l) *}\right] E\left[\varepsilon_{i s_{1}} \varepsilon_{n s_{2}}\right] \\
& \leq C \sum_{i=1}^{N} \sum_{n=1}^{N} \sum_{t_{1}=1}^{T} \sum_{t_{2}=1}^{T} \sum_{s_{1}=1}^{T} K_{s_{1}, 0}\left(\tau_{t_{1}}\right) K_{s_{1}, 0}\left(\tau_{t_{2}}\right)\left|E\left[x_{i s_{1}}^{(l) *} x_{n s_{1}}^{(l) *}\right]\right| \cdot\left|E\left[\varepsilon_{i s_{1}} \varepsilon_{n s_{1}}\right]\right| \\
& +C \sum_{i=1}^{N} \sum_{n=1}^{N} \sum_{t_{1}=1}^{T} \sum_{t_{2}=1}^{T} \sum_{s_{1}=1}^{T} \sum_{s_{2}=s_{1}+1}^{T} K_{s_{1}, 0}\left(\tau_{t_{1}}\right) K_{s_{2}, 0}\left(\tau_{t_{2}}\right)\left|E\left[x_{i s_{1}}^{(l) *} x_{n s_{1}}^{(l) *}\right]\right| \cdot\left|E\left[\varepsilon_{i s_{1}} \varepsilon_{n s_{1}}\right]\right|
\end{aligned}
$$




$$
\begin{aligned}
& +C \sum_{i=1}^{N} \sum_{n=1}^{N} \sum_{t_{1}=1}^{T} \sum_{t_{2}=1}^{T} \sum_{s_{1}=1}^{T} \sum_{s_{2}=1}^{s_{1}-1} K_{s_{1}, 0}\left(\tau_{t_{1}}\right) K_{s_{2}, 0}\left(\tau_{t_{2}}\right)\left|E\left[x_{i s_{1}}^{(l) *} x_{n s_{1}}^{(l) *}\right]\right| \cdot\left|E\left[\varepsilon_{i s_{1}} \varepsilon_{n s_{1}}\right]\right| \\
& =C \sum_{i=1}^{N} \sum_{n=1}^{N}\left(D_{i n, 4}+D_{i n, 5}+D_{i n, 6}\right) .
\end{aligned}
$$

For $D_{i n, 4}$,

$$
\begin{aligned}
& \left|D_{i n, 4}\right| \leq \sum_{t_{1}=1}^{T} \sum_{t_{2}=1}^{T} \sum_{s_{1}=1}^{T} K_{s_{1}, 0}\left(\tau_{t_{1}}\right) K_{s_{1}, 0}\left(\tau_{t_{2}}\right)\left|\operatorname{Cov}\left(x_{i 1}^{(l) *} \varepsilon_{i 1}, x_{n 1}^{(l) *} \varepsilon_{n 1}\right)\right| \\
& \leq C T^{3} h_{0}^{2} \alpha_{i j}(0)^{\delta /(4+\delta)}\left(E\left[\left|x_{i 1}^{(l) *}\right|^{2+\delta / 2}\right] E\left[\left|\varepsilon_{i 1}\right|^{2+\delta / 2}\right]\right)^{2 /(4+\delta)} \\
& \times\left(E\left[\left|x_{n, 1}^{(l) *}\right|^{2+\delta / 2}\right] E\left[\left|\varepsilon_{n, 1}\right|^{2+\delta / 2}\right]\right)^{2 /(4+\delta)} \leq C T^{3} h_{0}^{2} \alpha_{i j}(0)^{\delta /(4+\delta)} .
\end{aligned}
$$

Therefore, we have $\sum_{i=1}^{N} \sum_{n=1}^{N} D_{i n, 4}=O\left(N T^{3} h_{0}^{2}\right)$.

Analogously, we can show that $\sum_{i=1}^{N} \sum_{n=1}^{N} D_{i n, 5}=O\left(N T^{3} h_{0}^{2}\right)$ and $\sum_{i=1}^{N} \sum_{n=1}^{N} D_{i n, 6}=O\left(N T^{3} h_{0}^{2}\right)$. Therefore, Lemma A.1.(2) holds.

(3) Analogously to Lemma A.1.(1), we can easily show that

$$
\sum_{t=1}^{T}\left\|\sum_{i=1}^{N}\left(\mathbf{x}_{i t}^{*} \mathbf{x}_{i t}^{* \top}-E\left[\mathbf{x}_{i t}^{*} \mathbf{x}_{i t}^{* \top}\right]\right)\right\|=O_{P}(\sqrt{N T}) .
$$

We have

$$
\begin{aligned}
& \sup _{\tau \in(0,1)}\left\|\frac{1}{N T h_{0}} \sum_{i=1}^{N} \sum_{t=1}^{T} K_{t, 0}(\tau)\left(\mathbf{x}_{i t}^{*} \mathbf{x}_{i t}^{* \top}-E\left[\mathbf{x}_{i t}^{*} \mathbf{x}_{i t}^{* \top}\right]\right)\right\| \leq \frac{C}{N T h_{0}} \sum_{t=1}^{T}\left\|\sum_{i=1}^{N}\left(\mathbf{x}_{i t}^{*} \mathbf{x}_{i t}^{* \top}-E\left[\mathbf{x}_{i t}^{*} \mathbf{x}_{i t}^{* \top}\right]\right)\right\| \\
& =o_{P}(1) .
\end{aligned}
$$

Therefore, we have Lemma A.1.(3) holds.

(4) For $\widehat{m}_{X, i}^{(l)}(\tau)$, recall that

$$
\widehat{m}_{X, i}^{(l)}(\tau)=[1,0]\left(\mathbf{M}_{b}^{\top}(\tau) \mathbf{W}_{b}(\tau) \mathbf{M}_{b}(\tau)\right)^{-1} \mathbf{M}_{b}^{\top}(\tau) \mathbf{W}_{b}(\tau) \mathbf{x}_{i .}^{(l)}
$$

By the definition of $\mathbf{M}_{b}(\tau)$ and $\mathbf{W}_{b}(\tau)$, we can see that $\mathbf{M}_{b}^{\top}(\tau) \mathbf{W}_{b}(\tau) \mathbf{M}_{b}(\tau)$ is a deterministic function of $\tau$. Then it is sufficient to show that

$$
\sup _{\tau \in(0,1)}\left\|\frac{1}{T b} \sum_{t=1}^{T} K_{t, m}(\tau) x_{i t}^{(l)}-E\left[x_{i t}^{(l)} \mid \tau_{t}=\tau\right]\right\|=o_{P}(1),
$$

for $m=0,1$.

Following analogous arguments to Lemma A.1.(3), we can show that (B.5) holds.

\section{Proof of Lemma A.2}

In this lemma, we derive asymptotic distributions with the conventional large-block and small-block technique. By partitioning the set $1,2, \cdots, T$ into $2 \kappa_{T}+1$ subsets with large block with size $l_{T}$, small 
block with size $s_{T}$ and the remaining set with size $T-\kappa_{T}\left(l_{T}+s_{T}\right)$. We can choose $l_{T}, s_{T}$ to make the following conditions hold:

$$
s_{T} \rightarrow \infty, \quad \frac{s_{T}}{l_{T}} \rightarrow 0, \quad \frac{l_{T}}{T} \rightarrow 0, \quad \text { and } \quad \kappa_{T}=\left[\frac{T}{l_{T}+s_{T}}\right]=O\left(s_{T}\right),
$$

where $[m]$ operator define the largest integer which is bounded by $m$.

Let $\boldsymbol{\nu}_{N T, t}=\frac{1}{\sqrt{N T h_{0}}} \sum_{i=1}^{N} K_{t, 0}(\tau) \widetilde{\mathbf{x}}_{i t}^{*} \varepsilon_{i t}$. We introduce the following notation:

$$
\widetilde{\boldsymbol{\nu}}_{\rho}=\sum_{t=(\rho-1)\left(l_{T}+s_{T}\right)+1}^{\rho l_{T}+(\rho-1) s_{T}} \boldsymbol{\nu}_{N T, t}, \quad \widehat{\boldsymbol{\nu}}_{\rho}=\sum_{t=\rho l_{T}+(\rho-1) s_{T}+1}^{\rho\left(l_{T}+s_{T}\right)} \boldsymbol{\nu}_{N T, t},
$$

and $\overline{\boldsymbol{\nu}}=\sum_{t=\kappa_{T}\left(l_{T}+s_{T}\right)+1}^{T} \boldsymbol{\nu}_{N T, t}$ for $\rho=1, \ldots, \kappa_{T}$.

It is clear to see that

$$
\sum_{t=1}^{T} \boldsymbol{\nu}_{N T, t}=\sum_{\rho=1}^{\kappa_{T}} \widetilde{\boldsymbol{\nu}}_{\rho}+\sum_{\rho=1}^{\kappa_{T}} \widehat{\boldsymbol{\nu}}_{\rho}+\overline{\boldsymbol{\nu}}
$$

By the properties of $\alpha$-mixing processes and following analogous steps to (A.6) in Chen et al. (2012a), we can show that

$$
\left\|\sum_{\rho=1}^{\kappa_{T}} \widehat{\boldsymbol{\nu}}_{\rho}\right\|=O_{P}\left(\sqrt{\frac{\kappa_{T} s_{T}}{T}}\right)
$$

and

$$
\|\overline{\boldsymbol{\nu}}\|=O_{P}\left(\sqrt{\frac{T-\kappa_{T}\left(l_{T}+s_{T}\right)}{T}}\right) .
$$

For $\sum_{\rho=1}^{\kappa_{T}} \widetilde{\boldsymbol{\nu}}_{\rho}$, by Proposition 2.6 in Fan and Yao (2003), we have

$$
\left|E\left[\exp \left\{i t \sum_{\rho=1}^{\kappa_{T}} \widetilde{\nu}_{\rho}\right\}\right]-\prod_{\rho=1}^{\kappa_{T}} E\left[\exp \left\{i t \widetilde{\nu}_{\rho}\right\}\right]\right| \leq 16\left(\kappa_{T}-1\right) \alpha\left(s_{T}\right)=o(1) .
$$

The covariance of the large blocks is given by

$$
\begin{aligned}
& \sum_{\rho=1}^{\kappa_{T}} \operatorname{Cov}\left(\widetilde{\boldsymbol{\nu}}_{\rho}\right)=\sum_{\rho=1}^{\kappa_{T}} \operatorname{Cov}\left(\sum_{t=(\rho-1)\left(l_{T}+s_{T}\right)+1}^{\rho l_{T}+(\rho-1) s_{T}} \boldsymbol{\nu}_{N T, t}\right) \\
& =\frac{1}{N T h_{0}} \sum_{\rho=1}^{\kappa_{T}} \sum_{t=(\rho-1)\left(l_{T}+s_{T}\right)+1}^{\rho l_{T}+(\rho-1) s_{T}} \sum_{s=(\rho-1)\left(l_{T}+s_{T}\right)+1}^{\rho l_{T}+(\rho-1) s_{T}} \sum_{i=1}^{N} \sum_{n=1}^{N} K_{t, 0}(\tau) K_{s, 0}(\tau) \operatorname{Cov}\left(\mathbf{x}_{i t}^{*} \varepsilon_{i t}, \mathbf{x}_{n s}^{*} \varepsilon_{n s}\right) .
\end{aligned}
$$

It implies that

$$
\sum_{\rho=1}^{\kappa_{T}} \operatorname{Cov}\left(\widetilde{\boldsymbol{\nu}}_{\rho}\right)=\boldsymbol{\Sigma}_{\beta}^{0}+o(1)
$$

where $\boldsymbol{\Sigma}_{\beta}^{0}$ is defined in Assumption 5. It implies that the Feller condition is satisfied.

For any $\varepsilon>0$, we have

$$
\begin{aligned}
& E\left[\left\|\widetilde{\boldsymbol{\nu}}_{\rho}\right\|^{2} I\left\{\left\|\widetilde{\boldsymbol{\nu}}_{\rho}\right\| \geq \varepsilon\right\}\right] \leq\left(E\left[\left\|\widetilde{\boldsymbol{\nu}}_{\rho}\right\|^{3}\right]\right)^{\frac{2}{3}}\left(\operatorname{Pr}\left[\left\|\widetilde{\boldsymbol{\nu}}_{\rho}\right\| \geq \varepsilon\right]\right)^{\frac{1}{3}} \\
& \leq \varepsilon^{-\frac{2}{3}}\left(E\left[\left\|\widetilde{\boldsymbol{\nu}}_{\rho}\right\|^{3}\right]\right)^{\frac{2}{3}}\left(E\left[\left\|\widetilde{\boldsymbol{\nu}}_{\rho}\right\|^{2}\right]\right)^{\frac{1}{3}}=O\left(\frac{l_{T}}{T}\right)^{\frac{1}{3}} O\left(\left(\frac{l_{T}}{T}\right)^{\frac{3}{2}}\right)^{\frac{2}{3}}=O\left(\left(\frac{l_{T}}{T}\right)^{\frac{4}{3}}\right),
\end{aligned}
$$


where the second last equality holds by a technical lemma in Theorem 4.1 of Shao and Yu (1996). It implies that

$$
\sum_{\rho=1}^{\kappa_{T}} E\left[\left\|\widetilde{\boldsymbol{\nu}}_{\rho}\right\|^{2} I\left\{\left\|\widetilde{\boldsymbol{\nu}}_{\rho}\right\| \geq \varepsilon\right\}\right]=O\left(\left(\frac{l_{T}}{T}\right)^{\frac{4}{3}} \kappa_{T}\right)=o(1)
$$

and therefore the Lindeberg condition is satisfied which completes the proof. Following analogous proof steps, we can show that Lemma A.2.(2) holds.

\section{Proof of Lemma A.3}

Recall that we have the following notation

$$
\widehat{\mathcal{P}}_{j t}^{(l)}\left(v_{j}\right)=N^{-1} \sum_{i=1}^{N} \mathcal{H}_{N, t}^{(l)}\left(\mathbf{V}_{i t}^{(-j)}\left(v_{j}\right)\right) W^{(-j)}\left(\mathbf{V}_{i t}^{(-j)}\right) w_{j}\left(v_{j}\right), \quad \mathcal{P}_{j t}^{(l)}\left(v_{j}\right)=E\left[\mathcal{H}_{t}^{(l)}\left(\mathbf{V}_{i t}^{(-j)}\left(v_{j}\right)\right) W^{(-j)}\left(\mathbf{V}_{i t}^{(-j)}\right)\right] w_{j}\left(v_{j}\right),
$$

where $\mathcal{H}_{N, t}^{(0)}(\mathbf{v})=\widehat{E}\left[\widehat{\widetilde{y}}_{i t} \mid \mathbf{V}_{i t}=\mathbf{v}\right], \mathcal{H}_{N, t}^{(l)}(\mathbf{v})=\widehat{E}\left[\widehat{\widetilde{x}}_{i t}^{(l)} \mid \mathbf{V}_{i t}=\mathbf{v}\right]$, for $l=1, \cdots, p$, are local linear estimators of $\mathcal{H}_{t}^{(0)}(\mathbf{v})=E\left[\widetilde{y}_{i t} \mid \mathbf{V}_{i t}=\mathbf{v}\right], \mathcal{H}_{t}^{(l)}(\mathbf{v})=E\left[\widetilde{x}_{i t}^{(l)} \mid \mathbf{V}_{i t}=\mathbf{v}\right]$, respectively.

We introduce the following approximation to $\mathcal{P}_{j t}^{(l)}\left(v_{j}\right)$ :

$$
\widetilde{\mathcal{P}}_{j t}^{(l)}\left(v_{j}\right)=N^{-1} \sum_{i=1}^{N} \mathcal{H}_{t}^{(l)}\left(\mathbf{V}_{i t}^{(-j)}\left(v_{j}\right)\right) W^{(-j)}\left(\mathbf{V}_{i t}^{(-j)}\right) w_{j}\left(v_{j}\right)
$$

Let $\widetilde{\mathcal{P}}_{j t}^{(l) c}\left(v_{j}\right)=\widetilde{P}_{j t}^{(l)}\left(v_{j}\right)-\mathcal{P}_{j t}^{(l)}\left(v_{j}\right)$ be the approximation error. By the law of large numbers, it is clear to see that

$$
\sup _{v_{j} \in\left[\underline{v}_{j}, \bar{v}_{j}\right]} \widetilde{P}_{j t}^{(l) c}\left(v_{j}\right)=O_{P}\left(\frac{1}{\sqrt{N}}\right)
$$

For $\widehat{\mathcal{P}}_{j t}^{(l)}\left(v_{j}\right)-\widetilde{P}_{j t}^{(l)}\left(v_{j}\right)$ with $v_{j} \in\left[\underline{v}_{j}, \bar{v}_{j}\right]$,

$$
\widehat{\mathcal{P}}_{j t}^{(l)}\left(v_{j}\right)-\widetilde{P}_{j t}^{(l)}\left(v_{j}\right)=\frac{1}{N} \sum_{i=1}^{N}\left(\mathcal{H}_{N, t}^{(l)}\left(\mathbf{V}_{i t}^{(-j)}\left(v_{j}\right)\right)-\mathcal{H}_{t}^{(l)}\left(\mathbf{V}_{i t}^{(-j)}\left(v_{j}\right)\right)\right) W^{(-j)}\left(\mathbf{V}_{i t}^{(-j)}\right) .
$$

Recall that we have the following notation for the local linear estimator $\mathcal{H}_{N, t}^{(l)}(\mathbf{v})$ :

$$
\begin{aligned}
& \mathcal{V}_{i t}(\mathbf{v})=\left(\frac{V_{1 i t}-v_{1}}{h_{1}}, \frac{V_{2 i t}-v_{2}}{h_{2}}, \ldots, \frac{V_{J i t}-v_{J}}{h_{J}}\right)^{\top}, \quad \mathcal{K}\left(\mathcal{V}_{i t}(\mathbf{v})\right)=\prod_{j=1}^{J} K\left(\frac{V_{j i t}-v_{j}}{h_{j}}\right) \\
& \mathcal{W}_{t}(\mathbf{v})=\operatorname{diag}\left(\mathcal{K}\left(\mathcal{V}_{1 t}(\mathbf{v})\right), \ldots, \mathcal{K}\left(\mathcal{V}_{N t}(\mathbf{v})\right)\right), \quad \mathcal{M}_{t}(\mathbf{v})=\left(\begin{array}{ccc}
1 & \ldots & 1 \\
\mathcal{V}_{1 t}(\mathbf{v}) & \ldots & \mathcal{V}_{N t}(\mathbf{v})
\end{array}\right)^{\top}
\end{aligned}
$$

where $h_{1}, \ldots, h_{J}$ are bandwidths.

With this notation, we have

$$
\begin{aligned}
& \mathcal{H}_{N, t}^{(l)}(\mathbf{v})=\left[1, \mathbf{0}_{1 \times J}\right]\left(\mathcal{M}_{t}^{\top}(\mathbf{v}) \mathcal{W}_{t}(\mathbf{v}) \mathcal{M}_{t}(\mathbf{v})\right)^{-1} \mathcal{M}_{t}^{\top}(\mathbf{v}) \mathcal{W}_{t}(\mathbf{v}) \widehat{\widetilde{\mathbf{x}}}_{t}^{(l)} \\
& \mathcal{H}_{N, t}^{(0)}(\mathbf{v})=\left[1, \mathbf{0}_{1 \times J}\right]\left(\mathcal{M}_{t}^{\top}(\mathbf{v}) \mathcal{W}_{t}(\mathbf{v}) \mathcal{M}_{t}(\mathbf{v})\right)^{-1} \mathcal{M}_{t}^{\top}(\mathbf{v}) \mathcal{W}_{t}(\mathbf{v}) \widehat{\widetilde{\mathbf{y}}}_{t}
\end{aligned}
$$


where $\widehat{\widetilde{\mathbf{x}}}_{t}^{(l)}=\left(\widehat{\widetilde{x}}_{1 t}^{(l)}, \cdots, \widehat{\widetilde{x}}_{N t}^{(l)}\right)^{\top}$ and $\widehat{\widetilde{\mathbf{y}}}_{t}^{(l)}=\left(\widehat{\widetilde{y}}_{1 t}^{(l)}, \cdots, \widehat{\widetilde{y}}_{N t}^{(l)}\right)^{\top}$.

Let $\mathbf{s}_{t}(\mathbf{v})=\left[1, \mathbf{0}_{1 \times J}\right]\left(\mathcal{M}_{t}^{\top}(\mathbf{v}) \mathcal{W}_{t}(\mathbf{v}) \mathcal{M}_{t}(\mathbf{v})\right)^{-1} \mathcal{M}_{t}^{\top}(\mathbf{v}) \mathcal{W}_{t}(\mathbf{v})$. By Taylor expansion, we have

$$
\begin{aligned}
& \widehat{\mathcal{P}}_{j t}^{(l)}\left(v_{j}\right)-\widetilde{P}_{j t}^{(l)}\left(v_{j}\right)=\frac{1}{N} \sum_{i=1}^{N}\left(\mathbf{s}_{t}\left(\mathbf{V}_{i t}^{(-j)}\left(v_{j}\right)\right) \mathbf{x}_{t}^{(l)}-\mathcal{H}_{t}^{(l)}\left(\mathbf{V}_{i t}^{(-j)}\left(v_{j}\right)\right)\right) W^{(-j)}\left(\mathbf{V}_{i t}^{(-j)}\right) \\
& =\frac{1}{N} \sum_{i=1}^{N} \mathbf{s}_{t}\left(\mathbf{V}_{i t}^{(-j)}\left(v_{j}\right)\right)\left(\widetilde{\mathcal{H}}_{t}^{(l)}\left(\mathbf{V}_{i t}^{(-j)}\left(v_{j}\right)\right)+o_{P}\left(\sum_{j=1}^{J} h_{j}^{2}\right)\right)\left(1+o_{P}(1)\right) W^{(-j)}\left(\mathbf{V}_{i t}^{(-j)}\right) \\
& +\frac{1}{N} \sum_{i=1}^{N} \mathbf{s}_{t}\left(\mathbf{V}_{i t}^{(-j)}\left(v_{j}\right)\right) \boldsymbol{\xi}_{t}^{(l)} W^{(-j)}\left(\mathbf{V}_{i t}^{(-j)}\right)=J_{N T, 1}\left(v_{j}\right)+J_{N T, 2}\left(v_{j}\right),
\end{aligned}
$$

where $\boldsymbol{\xi}_{t}^{(l)}=\left(\xi_{1 t}^{(l)}, \ldots, \xi_{N t}^{(l)}\right)^{\top}$ with $\xi_{i t}^{(l)}=x_{i t}^{(l)}-\mathcal{H}_{t}^{(l)}\left(\mathbf{V}_{i t}\right), \widetilde{\mathcal{H}}_{t}^{(l)}(\mathbf{v})=\left(\widetilde{\mathcal{H}}_{1 t}^{(l)}(\mathbf{v}), \ldots, \widetilde{\mathcal{H}}_{N t}^{(l)}(\mathbf{v})\right)^{\top}$ and

$$
\widetilde{\mathcal{H}}_{i t}^{(l)}(\mathbf{v})=\mathcal{V}_{i t}^{\top}(\mathbf{v}) \mathcal{H}_{H, t}^{(l) \prime}(\mathbf{v}) \mathcal{V}_{i t}(\mathbf{v})=\sum_{j=1}^{J} \sum_{k=1}^{J}\left(\frac{V_{j i t}-v_{j}}{h_{j}}\right)\left(\frac{V_{k i t}-v_{k}}{h_{k}}\right) \frac{\partial^{2} \mathcal{H}_{t}^{(l)}(\mathbf{v})}{\partial v_{j} v_{k}} h_{j} h_{k},
$$

where the $(j, k)$-th element in $\mathcal{H}_{H, t}^{(l) \prime}(\mathbf{v})$ is $\frac{\partial^{2} \mathcal{H}_{t}^{(l)}(\mathbf{v})}{\partial v_{j} v_{k}} h_{j} h_{k}$.

Note that the rates of convergence for $J_{N T, 1}\left(v_{j}\right)$ and $J_{N T, 2}\left(v_{j}\right)$ are not related to the weight function $W^{(-j)}\left(\mathbf{v}^{(-j)}\right)$. Without loss of generality, we assume $W^{(-j)}\left(\mathbf{v}^{(-j)}\right)=1$ for notational simplicity. By Lemma B.1, it is clear to see that

$$
\begin{aligned}
& J_{N T, 1}\left(v_{j}\right)=\frac{1}{N} \sum_{i=1}^{N} p_{V}^{-1}\left(\mathbf{V}_{i t}^{(-j)}\left(v_{j}\right)\right)\left(\frac{1}{N H_{J}} \sum_{n=1}^{N} \mathcal{K}\left(\mathcal{V}_{n t}\left(\mathbf{V}_{i t}^{(-j)}\left(v_{j}\right)\right)\right) \widetilde{\mathcal{H}}_{n t}^{(l)}\left(\mathbf{V}_{i t}^{(-j)}\left(v_{j}\right)\right)\right)\left(1+o_{P}(1)\right) \\
& =\frac{1}{N h_{j}} \sum_{n=1}^{N} p_{V}^{-1}\left(\mathbf{V}_{n t}^{(-j)}\left(v_{j}\right)\right)\left(K\left(\frac{V_{j n t}-v_{j}}{h_{j}}\right)\left(\frac{V_{j n t}-v_{j}}{h_{j}}\right)^{2} \frac{\partial^{2} \mathcal{H}_{t}^{(l)}\left(\mathbf{V}_{n t}^{(-j)}\left(v_{j}\right)\right)}{\partial v_{j}^{2}} h_{j}^{2}\right) \\
& +\frac{1}{N h_{j}} \sum_{n=1}^{N} \sum_{k \neq j} \mu_{2} p_{V}^{-1}\left(\mathbf{V}_{n t}^{(-j)}\left(v_{j}\right)\right) p_{V}^{(-j)}\left(\mathbf{V}_{n t}^{(-j)}\right)\left(K\left(\frac{V_{j n t}-v_{j}}{h_{j}}\right) \frac{\partial^{2} \mathcal{H}_{t}^{(l)}\left(\mathbf{V}_{n t}^{(-j)}\left(v_{j}\right)\right)}{\partial v_{k}^{2}} h_{k}^{2}\right) \\
& +o_{P}\left(\sum_{k=1}^{J} h_{k}^{2}\right)=O_{P}\left(\sum_{k=1}^{J} h_{k}^{2}\right) .
\end{aligned}
$$

It implies that for $v_{j} \in\left[\underline{v}_{j}, \bar{v}_{j}\right], J_{N T, 1}\left(v_{j}\right)=o_{P}(1)$.

In addition, since the kernel function $K(\cdot)$ is Lipschitz continuous, for any $\widetilde{v}_{j} \in\left[\underline{v}_{j}, \bar{v}_{j}\right]$, there exists $M_{0}$ such that

$$
\left|K\left(\frac{V_{j n t-\widetilde{v}_{j}}}{h_{j}}\right)-K\left(\frac{V_{j n t}-v_{j}}{h_{j}}\right)\right| \leq \frac{M_{0}}{h_{j}}\left|\widetilde{v}_{j}-v_{j}\right| .
$$

Therefore, we have

$$
\begin{aligned}
& \| \frac{1}{N h_{j}} \sum_{n=1}^{N} p_{V}^{-1}\left(\mathbf{V}_{n t}^{(-j)}\left(\widetilde{v}_{j}\right)\right)\left(K\left(\frac{V_{j n t}-\widetilde{v}_{j}}{h_{j}}\right)\left(\frac{V_{j n t}-\widetilde{v}_{j}}{h_{j}}\right)^{2} \frac{\partial^{2} \mathcal{H}_{t}^{(l)}\left(\mathbf{V}_{n t}^{(-j)}\left(\widetilde{v}_{j}\right)\right)}{\partial v_{j}^{2}} h_{j}^{2}\right) \\
& -\frac{1}{N h_{j}} \sum_{n=1}^{N} p_{V}^{-1}\left(\mathbf{V}_{n t}^{(-j)}\left(v_{j}\right)\right)\left(K\left(\frac{V_{j n t}-v_{j}}{h_{j}}\right)\left(\frac{V_{j n t}-v_{j}}{h_{j}}\right)^{2} \frac{\partial^{2} \mathcal{H}_{t}^{(l)}\left(\mathbf{V}_{n t}^{(-j)}\left(v_{j}\right)\right)}{\partial v_{j}^{2}} h_{j}^{2}\right) \| \\
& \leq M_{1}\left|\widetilde{v}_{j}-v_{j}\right|,
\end{aligned}
$$

where

$$
M_{1}=\sup _{v_{j} \in\left[\underline{v}_{j}, \bar{v}_{j}\right]} \frac{1}{N h_{j}} \sum_{n=1}^{N}\left(\frac{M_{0}}{h_{j}} p_{V}^{-1}\left(\mathbf{V}_{n t}^{(-j)}\left(v_{j}\right)\right)\left(\frac{V_{j n t}-v_{j}}{h_{j}}\right)^{2} \frac{\partial^{2} \mathcal{H}_{t}^{(l)}\left(\mathbf{V}_{n t}^{(-j)}\left(v_{j}\right)\right)}{\partial v_{j}^{2}} h_{j}^{2}+\right.
$$




$$
\begin{aligned}
& \left.\left.K\left(\frac{V_{j n t}-\widetilde{v}_{j}}{h_{j}}\right) \frac{\partial}{\partial v_{j}}\left(p_{V}^{-1}\left(\mathbf{V}_{n t}^{(-j)}\left(v_{j}\right)\right)\left(\frac{V_{j n t}-v_{j}}{h_{j}}\right)^{2} \frac{\partial^{2} \mathcal{H}_{t}^{(l)}\left(\mathbf{V}_{n t}^{(-j)}\left(v_{j}\right)\right)}{\partial v_{j}^{2}}\right)\right|_{v_{j}=\bar{v}_{j}} h_{j}^{2}\right) \\
& =O_{P}(1),
\end{aligned}
$$

for some $\bar{v}_{j} \in\left(v_{j}, \widetilde{v}_{j}\right)$ or $\bar{v}_{j} \in\left(\widetilde{v}_{j}, v_{j}\right)$.

For the second term in $J_{N T, 1}\left(v_{j}\right)$, for each $k \neq j$, we have

$$
\begin{aligned}
& \| \frac{1}{N h_{j}} \sum_{n=1}^{N} \mu_{2} p_{V}^{-1}\left(\mathbf{V}_{n t}^{(-j)}\left(\widetilde{v}_{j}\right)\right) p_{V}^{(-j)}\left(\mathbf{V}_{n t}^{(-j)}\right)\left(K\left(\frac{V_{j n t}-\widetilde{v}_{j}}{h_{j}}\right) \frac{\partial^{2} \mathcal{H}_{t}^{(l)}\left(\mathbf{V}_{n t}^{(-j)}\left(\widetilde{v}_{j}\right)\right)}{\partial v_{k}^{2}} h_{k}^{2}\right) \\
& -\frac{1}{N h_{j}} \sum_{n=1}^{N} \mu_{2} p_{V}^{-1}\left(\mathbf{V}_{n t}^{(-j)}\left(v_{j}\right)\right) p_{V}^{(-j)}\left(\mathbf{V}_{n t}^{(-j)}\right)\left(K\left(\frac{V_{j n t}-v_{j}}{h_{j}}\right) \frac{\partial^{2} \mathcal{H}_{t}^{(l)}\left(\mathbf{V}_{n t}^{(-j)}\left(v_{j}\right)\right)}{\partial v_{k}^{2}} h_{k}^{2}\right) \| \\
& \leq M_{2} \mid \widetilde{v}_{j}-v_{j} \|,
\end{aligned}
$$

where

$$
\begin{aligned}
& M_{2}=\sup _{v_{j} \in\left[\underline{v}_{j}, \bar{v}_{j}\right]} \frac{1}{N h_{j}} \sum_{n=1}^{N}\left(\frac{M_{0}}{h_{j}} p_{V}^{-1}\left(\mathbf{V}_{n t}^{(-j)}\left(v_{j}\right)\right) p_{V}^{(-j)}\left(\mathbf{V}_{n t}^{(-j)}\right) \frac{\partial^{2} \mathcal{H}_{t}^{(l)}\left(\mathbf{V}_{n t}^{(-j)}\left(v_{j}\right)\right)}{\partial v_{k}^{2}} h_{k}^{2}+\right. \\
& \left.\left.K\left(\frac{V_{j n t}-\widetilde{v}_{j}}{h_{j}}\right) p_{V}^{(-j)}\left(\mathbf{V}_{n t}^{(-j)}\right) \frac{\partial}{\partial v_{j}}\left(p_{V}^{-1}\left(\mathbf{V}_{n t}^{(-j)}\left(v_{j}\right)\right) \frac{\partial^{2} \mathcal{H}_{t}^{(l)}\left(\mathbf{V}_{n t}^{(-j)}\left(v_{j}\right)\right)}{\partial v_{k}^{2}}\right)\right|_{v_{j}=\bar{v}_{j}} h_{k}^{2}\right) \\
& =O_{P}(1) .
\end{aligned}
$$

By Lemma A2 in Newey and Powell (2003), we have $\sup _{v_{j} \in\left[\underline{v}_{j}, \bar{v}_{j}\right]}\left\|J_{N T, 1}\left(v_{j}\right)\right\|=o_{P}(1)$.

Analogously, we have $\sup _{v_{j} \in\left[\underline{v}_{j}, \bar{v}_{j}\right]}\left\|J_{N T, 2}\left(v_{j}\right)\right\|=o_{P}(1)$. Therefore, we have

$$
\sup _{v_{j} \in\left[\underline{v}_{j}, \bar{v}_{j}\right]}\left|\widehat{\mathcal{P}}_{j t}^{(l)}\left(v_{j}\right)-\widetilde{P}_{j t}^{(l)}\left(v_{j}\right)\right|=o_{P}(1) .
$$

By (B.8) and (B.11), Lemma A.3 holds.

\section{Proof of Lemma A.4}

It is clear to see that

$$
\begin{aligned}
& \widehat{\mathcal{P}}_{j t}^{(0)}\left(v_{j}\right)-\mathcal{P}_{j t}^{(0)}\left(v_{j}\right)-\boldsymbol{\beta}^{\top}\left(\tau_{t}\right)\left(\widehat{\mathbf{P}}_{j t}^{V}\left(v_{j}\right)-\mathcal{P}_{j t}^{V}\left(v_{j}\right)\right)=\widehat{\mathcal{P}}_{j t}^{(0)}\left(v_{j}\right)-\widetilde{P}_{j t}^{(0)}\left(v_{j}\right)-\sum_{l=1}^{p} \beta_{l}\left(\tau_{t}\right)\left(\widehat{\mathcal{P}}_{j t}^{(l)}\left(v_{j}\right)-\widetilde{P}_{j t}^{(l)}\left(v_{j}\right)\right) \\
& +\widetilde{P}_{j t}^{(0) c}\left(v_{j}\right)-\sum_{l=1}^{p} \beta_{l}\left(\tau_{t}\right) \widetilde{P}_{j t}^{(l) c}\left(v_{j}\right),
\end{aligned}
$$

where $\widetilde{P}_{j t}^{(l)}\left(v_{j}\right)=N^{-1} \sum_{i=1}^{N} \mathcal{H}_{t}^{(l)}\left(\mathbf{V}_{i t}^{(-j)}\left(v_{j}\right)\right) W^{(-j)}\left(\mathbf{V}_{i t}^{(-j)}\right) w_{j}\left(v_{j}\right)$ and $\widetilde{P}_{j t}^{(l) c}\left(v_{j}\right)=\widetilde{P}_{j t}^{(l)}\left(v_{j}\right)-\mathcal{P}_{j t}^{(l)}\left(v_{j}\right)$.

Note that by Lemma B.4,

$$
\begin{aligned}
& \widetilde{P}_{j t}^{(0) c}\left(v_{j}\right)-\sum_{l=1}^{p} \beta_{l}^{\top}\left(\tau_{t}\right) \widetilde{P}_{j t}^{(l) c}\left(v_{j}\right)=T_{j t}^{(0) a}\left(v_{j}\right)-\sum_{l=1}^{p} \beta_{l}\left(\tau_{t}\right) T_{j t}^{(l) a}\left(v_{j}\right) \\
& =\frac{1}{N} \sum_{i=1}^{N} \sum_{k \neq j}\left(\gamma_{k t}\left(V_{k i t}\right) W^{(-j)}\left(\mathbf{V}_{i t}^{(-j)}\right)-E\left[\gamma_{k t}\left(V_{k i t}\right) W^{(-j)}\left(\mathbf{V}_{i t}^{(-j)}\right)\right]\right)=T_{j t}^{a}\left(v_{j}\right),
\end{aligned}
$$

where its $\ell$-th element, $T_{j t}^{(l) a}\left(v_{j}\right)$, is defined as

$$
T_{j t}^{(l) a}\left(v_{j}\right)=\left(N^{-1} \sum_{i=1}^{N} \mathcal{H}_{t}^{(l)}\left(\mathbf{V}_{i t}^{(-j)}\left(v_{j}\right)\right) W^{(-j)}\left(\mathbf{V}_{i t}^{(-j)}\right)-E\left[\mathcal{H}_{t}^{(l)}\left(\mathbf{V}_{i t}^{(-j)}\left(v_{j}\right)\right) W^{(-j)}\left(\mathbf{V}_{i t}^{(-j)}\right)\right]\right) w_{j}\left(v_{j}\right) .
$$


For the rest of the terms, we have

$$
\begin{aligned}
& \widehat{\mathcal{P}}_{j t}^{(0)}\left(v_{j}\right)-\widetilde{P}_{j t}^{(0)}\left(v_{j}\right)-\sum_{l=1}^{p} \beta_{l}^{\top}\left(\tau_{t}\right)\left(\widehat{\mathcal{P}}_{j t}^{(l)}\left(v_{j}\right)-\widetilde{P}_{j t}^{(l)}\left(v_{j}\right)\right) \\
& =\frac{1}{N} \sum_{i=1}^{N}\left(\mathbf{s}\left(\mathbf{V}_{i t}^{(-j)}\left(v_{j}\right)\right) \widetilde{\gamma}_{\cdot t}-\gamma_{t}\left(\mathbf{V}_{i t}^{(-j)}\left(v_{j}\right)\right)\right) W^{(-j)}\left(\mathbf{V}_{i t}^{(-j)}\right)+\frac{1}{N} \sum_{i=1}^{N} \mathbf{s}\left(\mathbf{V}_{i t}^{(-j)}\left(v_{j}\right)\right) \varepsilon_{\cdot t} W^{(-j)}\left(\mathbf{V}_{i t}^{(-j)}\right) \\
& +o_{P}\left(\sum_{j=1}^{J} h_{j}^{2}\right)=T_{j t}^{b *}\left(v_{j}\right)+T_{j t}^{c *}\left(v_{j}\right)+o_{P}\left(\sum_{j=1}^{J} h_{j}^{2}\right),
\end{aligned}
$$

where $\widetilde{\boldsymbol{\gamma}}_{\cdot t}=\left(\gamma_{t}\left(\mathbf{V}_{1}\right), \ldots, \gamma_{t}\left(\mathbf{V}_{n t}\right)\right)^{\top}$ and $\varepsilon_{\cdot t}=\left(\varepsilon_{1 t}, \ldots, \varepsilon_{N t}\right)^{\top}$.

Let $\gamma_{H, t}^{\prime \prime \top}(\mathbf{v})=\left(h_{1}^{2} \gamma_{1 t}^{\prime \prime}\left(v_{1}\right), \ldots, h_{J}^{2} \gamma_{J t}^{\prime \prime}\left(v_{1}\right)\right), \widetilde{\mathcal{U}}(v)=\left(\widetilde{\mathcal{U}}_{1}(v), \ldots, \widetilde{\mathcal{U}}_{N}(v)\right)^{\top}$ with

$$
\widetilde{\mathcal{U}}_{i t}(v)=\left(\left(\frac{V_{1 i t}-v_{1}}{h_{1}}\right)^{2}, \ldots,\left(\frac{V_{J i t}-v_{J}}{h_{J}}\right)^{2}\right)^{\top}
$$

For $T_{j t}^{b *}\left(v_{j}\right)$, by Taylor expansion and Lemma B.2,

$$
\begin{aligned}
& T_{j t}^{b *}\left(v_{j}\right)=\frac{1}{N} \sum_{i=1}^{N} \mathbf{s}\left(\mathbf{V}_{i t}^{(-j)}\left(v_{j}\right)\right) \widetilde{\mathcal{U}}\left(\mathbf{V}_{i t}^{(-j)}\left(v_{j}\right)\right)\left(\frac{1}{2} \gamma_{H, t}^{\prime \prime}\left(\mathbf{V}_{i t}^{(-j)}\left(v_{j}\right)\right)+o_{P}\left(\sum_{j=1}^{J} h_{j}^{2}\right)\right) W^{(-j)}\left(\mathbf{V}_{i t}^{(-j)}\right) \\
& =\frac{\mu_{2, j} h_{j}^{2}}{2} \gamma_{j t}^{\prime \prime}\left(v_{j}\right)+\sum_{k \neq j} \frac{\mu_{2, k} h_{k}^{2}}{2} E\left[\gamma_{k t}^{\prime \prime}\left(V_{k i t}\right) W^{(-j)}\left(\mathbf{V}_{i t}^{(-j)}\right)\right]+o_{P}\left(\sum_{j=1}^{J} h_{j}^{2}\right) \\
& =T_{j t}^{b}\left(v_{j}\right)+o_{P}\left(\sum_{j=1}^{J} h_{j}^{2}\right)
\end{aligned}
$$

where $T_{j t}^{b}\left(v_{j}\right)$ is defined by $T_{j t}^{b}\left(v_{j}\right)=b_{j t}^{*}\left(v_{j}\right) h_{j}^{2}+\sum_{k \neq j} \frac{\mu_{2, k} h_{k}^{2}}{2} E\left[\gamma_{k t}^{\prime \prime}\left(V_{k i t}\right) W^{(-j)}\left(\mathbf{V}_{i t}^{(-j)}\right)\right]$ as in Lemma B.4.

For $T_{j t}^{c *}\left(v_{j}\right)$, by Lemma B.1,

$$
\begin{aligned}
& T_{j t}^{c *}\left(v_{j}\right)=\frac{1}{N} \sum_{i=1}^{N} p_{V}^{-1}\left(\mathbf{V}_{i t}^{(-j)}\left(v_{j}\right)\right)\left(\frac{1}{N H_{J}} \sum_{n=1}^{N} \mathcal{K}\left(\mathcal{V}_{n t}\left(\mathbf{V}_{i t}^{(-j)}\left(v_{j}\right)\right)\right) \varepsilon_{n t}\right) W^{(-j)}\left(\mathbf{V}_{i t}^{(-j)}\right) \\
& \left(1+o_{P}(1)\right) \\
& =\frac{1}{N h_{j}} \sum_{n=1}^{N} K\left(\frac{V_{j n t}-v_{j}}{h_{j}}\right) p_{V}^{-1}\left(\mathbf{V}_{n t}^{(-j)}\left(v_{j}\right)\right) p_{V}^{(-j)}\left(\mathbf{V}_{n t}^{(-j)}\right) W^{(-j)}\left(\mathbf{V}_{n t}^{(-j)}\right) \varepsilon_{n t}+o_{p}\left(\frac{1}{\sqrt{N h_{j}}}\right) \\
& =T_{j t}^{c}\left(v_{j}\right)+o_{p}\left(\frac{1}{\sqrt{N h_{j}}}\right),
\end{aligned}
$$

where $T_{j t}^{c}\left(v_{j}\right)=\frac{1}{N h_{j}} \sum_{n=1}^{N} K\left(\frac{V_{j n t}-v_{j}}{h_{j}}\right) p_{V}^{-1}\left(\mathbf{V}_{n t}^{(-j)}\left(v_{j}\right)\right) p_{V}^{(-j)}\left(\mathbf{V}_{n t}^{(-j)}\right) W^{(-j)}\left(\mathbf{V}_{n t}^{(-j)}\right) \varepsilon_{n t}$.

By (B.12), (B.13) and (B.14), we have

$$
\widehat{\mathcal{P}}_{j t}^{(0)}\left(v_{j}\right)-\mathcal{P}_{j t}^{(0)}\left(v_{j}\right)-\boldsymbol{\beta}^{\top}\left(\tau_{t}\right)\left(\widehat{\mathbf{P}}_{j t}^{V}\left(v_{j}\right)-\mathbf{P}_{j t}^{V}\left(v_{j}\right)\right)=T_{j t}^{a}+T_{j t}^{b}\left(v_{j}\right)+T_{j t}^{c}\left(v_{j}\right)+o_{P}\left(\sum_{j=1}^{J} h_{j}^{2}\right)+o_{P}\left(\frac{1}{\sqrt{N h_{j}}}\right) .
$$

For $T_{j t}^{c}\left(v_{j}\right)$, we have $E\left[\sum_{i=1}^{N} \sum_{t=1}^{T} T_{j t}^{c}\left(V_{j i t}\right)\right]=0$.

In addition, it is shown that

$$
\begin{aligned}
& E\left[\left|\sum_{i=1}^{N} \sum_{t=1}^{T} T_{j t}^{c}\left(V_{j i t}\right)\right|^{2}\right]=\sum_{i=1}^{N} \sum_{m=1}^{N} \sum_{t=1}^{T} \sum_{s=1}^{T} E\left[T_{j t}^{c}\left(V_{j i t}\right) T_{j s}^{c}\left(V_{j m s}\right)\right] \\
& \leq C \frac{1}{N^{2} h_{j}^{2}} \sum_{t=1}^{T} \sum_{s=1}^{T} \sum_{i=1}^{N} \sum_{m=1}^{N} \sum_{n_{1}=1}^{N} \sum_{n_{2}=1}^{N} E\left[K\left(\frac{V_{j n_{1} t}-V_{j i t}}{h_{j}}\right) K\left(\frac{V_{j n_{2} s}-V_{j m t}}{h_{j}}\right)\right]\left|E\left[\varepsilon_{n_{1} t} \varepsilon_{n_{2} s}\right]\right|
\end{aligned}
$$




$$
\begin{aligned}
& \leq C \sum_{t=1}^{T} \sum_{s=1}^{T} \sum_{n_{1}=1}^{N} \sum_{n_{2}=1}^{N}\left|E\left[\varepsilon_{n_{1} t} \varepsilon_{n_{2} s}\right]\right| \\
& \leq C T \sum_{n_{1}=1}^{N} \sum_{n_{2}=1}^{N} \sum_{s=1}^{T-1} \alpha_{n_{1} n_{2}}(s)^{\delta /(4+\delta)}\left(E\left[\left|\varepsilon_{n_{1} 1}\right|^{2+\delta / 2}\right] E\left[\left|\varepsilon_{n_{2}, 1+s}\right|^{2+\delta / 2}\right]\right)^{2 /(4+\delta)}=O(N T) .
\end{aligned}
$$

Therefore, we have shown that $\sum_{i=1}^{N} \sum_{t=1}^{T} T_{j t}^{c}\left(V_{j i t}\right)=O_{P}(\sqrt{N T})$.

\section{Proof of Lemma A.5}

(1) We introduce the following notation:

$$
\begin{aligned}
& \widetilde{\boldsymbol{\gamma}}_{\cdot t}=\left(\gamma_{t}\left(\mathbf{V}_{1 t}\right), \gamma_{t}\left(\mathbf{V}_{2 t}\right), \ldots, \gamma_{t}\left(\mathbf{V}_{N t}\right)\right)^{\top}, \quad \widetilde{\gamma}_{t}^{(j)}=\left(\gamma_{j t}\left(V_{j 1 t}\right), \gamma_{j t}\left(V_{j 2 t}\right), \ldots, \gamma_{j t}\left(V_{j N t}\right)\right)^{\top} \\
& \mathbf{s}_{t}(\mathbf{v})=\left[1, \mathbf{0}_{1 \times J}\right]\left(\mathcal{M}_{t}^{\top}(\mathbf{v}) \mathcal{W}_{t}(\mathbf{v}) \mathcal{M}_{t}(\mathbf{v})\right)^{-1} \mathcal{M}_{t}^{\top}(\mathbf{v}) \mathcal{W}_{t}(\mathbf{v}) .
\end{aligned}
$$

For each $j$,

$$
\begin{aligned}
& \sum_{i=1}^{N} \sum_{t=1}^{T}\left(\widehat{\mathcal{P}}_{j t}^{\gamma}\left(V_{j i t}\right)-\gamma_{j t}\left(V_{j i t}\right)\right) \\
& =\sum_{i=1}^{N} \sum_{t=1}^{T}\left(\frac{1}{N} \sum_{n=1}^{N} \mathcal{H}_{N, t}^{\gamma}\left(\mathbf{V}_{n t}^{(-j)}\left(V_{j i t}\right)\right) W^{(-j)}\left(\mathbf{V}_{n t}^{(-j)}\right) w_{j}\left(V_{j i t}\right)-\gamma_{j t}\left(V_{j i t}\right)\right) \\
& =\sum_{i=1}^{N} \sum_{t=1}^{T}\left(\frac{1}{N} \sum_{n=1}^{N} W^{(-j)}\left(\mathbf{V}_{n t}^{(-j)}\right) w_{j}\left(V_{j i t}\right) \mathbf{s}_{t}\left(\mathbf{V}_{n t}^{(-j)}\left(V_{j i t}\right)\right) \widetilde{\gamma}_{\cdot t}-\gamma_{j t}\left(V_{j i t}\right)\right) \\
& =\sum_{i=1}^{N} \sum_{t=1}^{T}\left(\frac{1}{N} \sum_{n=1}^{N} W^{(-j)}\left(\mathbf{V}_{n t}^{(-j)}\right) w_{j}\left(V_{j i t}\right) \mathbf{s}_{t}\left(\mathbf{V}_{n t}^{(-j)}\left(V_{j i t}\right)\right) \widetilde{\gamma}_{t}^{(j)}-\gamma_{j t}\left(V_{j i t}\right)\right) \\
& +\sum_{i=1}^{N} \sum_{t=1}^{T} \sum_{k \neq j}\left(\frac{1}{N} \sum_{n=1}^{N} W^{(-j)}\left(\mathbf{V}_{n t}^{(-j)}\right) w_{j}\left(V_{j i t}\right) \mathbf{s}_{t}\left(\mathbf{V}_{n t}^{(-j)}\left(V_{j i t}\right)\right) \widetilde{\gamma}_{t}^{(k)}\right)=D_{N T, 1}+D_{N T, 2 .} .
\end{aligned}
$$

Let $\widehat{\Omega}_{t}^{C}(\mathbf{v})=\left(N H_{J}\right)^{-1} \mathcal{M}_{t}^{\top}(\mathbf{v}) \mathcal{W}_{t}(\mathbf{v}) \mathcal{M}_{t}(\mathbf{v})$. For simplicity, we assume $W^{(-j)}\left(\mathbf{V}_{i t}^{(-j)}\right) \equiv 1$ and $w_{j}\left(V_{j i t}\right) \equiv 1$ in the proof of this lemma. The general case can then be proved analogously. For $D_{N T, 1}$,

$$
\begin{aligned}
D_{N T, 1} & =\sum_{i=1}^{N} \sum_{t=1}^{T}\left(\frac{1}{N^{2} H_{J}} \sum_{n=1}^{N}\left[1, \mathbf{0}_{1 \times J}\right] \widehat{\boldsymbol{\Omega}}_{t}^{C}\left(\mathbf{V}_{n t}^{(-j)}\left(V_{j i t}\right)\right)^{-1} \mathcal{M}_{t}^{\top}\left(\mathbf{V}_{n t}^{(-j)}\left(V_{j i t}\right)\right) \mathcal{W}_{t}\left(\mathbf{V}_{n t}^{(-j)}\left(V_{j i t}\right)\right) \widetilde{\gamma}_{t}^{(j)}-\gamma_{j t}\left(V_{j i t}\right)\right) \\
& =\sum_{i=1}^{N} \sum_{t=1}^{T}\left(\frac{1}{N^{2} H_{J}} \sum_{n=1}^{N}\left[1, \mathbf{0}_{1 \times J}\right] \widehat{\boldsymbol{\Omega}}_{t}^{C}\left(\mathbf{V}_{n t}^{(-j)}\left(V_{j i t}\right)\right)^{-1}\left(D_{N T, 11}^{(i, n, t)}, D_{N T, 12}^{(i, n, t)}\right)^{\top}\right),
\end{aligned}
$$

where $D_{N T, 11}^{(i, n, t)}=\sum_{m=1}^{N} K\left(\frac{V_{j m t}-V_{j i t}}{h_{j}}\right) \prod_{k \neq j} K\left(\frac{V_{k m t}-V_{k n t}}{h_{k}}\right)\left(\gamma_{j t}\left(V_{j m t}\right)-\gamma_{j t}\left(V_{j i t}\right)\right)$ and

$$
D_{N T, 12}^{(i, n, t)}=\sum_{m=1}^{N} K\left(\frac{V_{j m t}-V_{j i t}}{h_{j}}\right)\left(\frac{V_{j m t}-V_{j i t}}{h_{j}}\right) \prod_{k \neq j}\left(K\left(\frac{V_{k m t}-V_{k n t}}{h_{k}}\right)\left(\frac{V_{j m t}-V_{j i t}}{h_{j}}\right)\right)\left(\gamma_{j t}\left(V_{j m t}\right)-\gamma_{j t}\left(V_{j i t}\right)\right) .
$$

By Lemmas B.1 and B.2, we have

$$
D_{N T, 1}=\sum_{i=1}^{N} \sum_{t=1}^{T}\left(\frac{1}{N^{2} H_{J}} \sum_{n=1}^{N} p_{V}^{-1}\left(\mathbf{V}_{n t}^{(-j)}\left(V_{j i t}\right)\right) D_{N T, 11}^{(i, n, t)}\right)\left(1+o_{P}(1)\right)=O_{P}\left(N T h_{j}^{2}\right) .
$$

For $D_{N T, 2}$, we have

$$
\begin{aligned}
& D_{N T, 2}=\sum_{i=1}^{N} \sum_{t=1}^{T} \sum_{k \neq j}\left(\frac{1}{N^{2} H_{J}} \sum_{n=1}^{N}\left[1, \mathbf{0}_{1 \times J}\right] \widehat{\mathbf{\Omega}}_{t}^{C}\left(\mathbf{V}_{n t}^{(-j)}\left(V_{j i t}\right)\right)^{-1}\left(D_{N T, 21}^{(k, i, n, t)}, D_{N T, 22}^{(k, i, n, t)}\right)^{\top}\right) \\
& =\sum_{i=1}^{N} \sum_{t=1}^{T} \sum_{k \neq j}\left(\frac{1}{N^{2} H_{J}} \sum_{n=1}^{N} p_{V}^{-1}\left(\mathbf{V}_{n t}^{(-j)}\left(V_{j i t}\right)\right) D_{N T, 21}^{(k, i, n, t)}\right)\left(1+o_{P}(1)\right),
\end{aligned}
$$


where

$$
\begin{aligned}
& D_{N T, 21}^{(k, i, n, t)}=\sum_{m=1}^{N} K\left(\frac{V_{j m t}-V_{j i t}}{h_{j}}\right) \prod_{l \neq j} K\left(\frac{V_{l m t}-V_{l n t}}{h_{l}}\right) \gamma_{j t}\left(V_{k m t}\right), \\
& D_{N T, 22}^{(k, i, n, t)}=\sum_{m=1}^{N} K\left(\frac{V_{j m t}-V_{j i t}}{h_{j}}\right)\left(\frac{V_{j m t}-V_{j i t}}{h_{j}}\right) \prod_{l \neq j}\left(K\left(\frac{V_{l m t}-V_{l n t}}{h_{l}}\right)\left(\frac{V_{j m t}-V_{j i t}}{h_{j}}\right)\right) \gamma_{j t}\left(V_{k m t}\right) .
\end{aligned}
$$

By Lemma B.2, we have $D_{N T, 2}=O_{P}\left(N T \sum_{k \neq j} h_{k}^{2}\right)$. Therefore, Lemma A.5.(1) holds.

(2) As can be seen from the proof of Lemma A.5.(1), the convergence of $\sum_{i=1}^{N} \sum_{t=1}^{T} \mathcal{G}_{i t}$ is a result of the kernel estimation of $\gamma_{j t}\left(v_{j}\right)$ in $\mathcal{G}_{i t}$. Therefore, the presence of $\mathbf{x}_{i t}^{*}$ has no effect on the rate of convergence of $\sum_{i=1}^{N} \sum_{t=1}^{T} K_{t, 0}(\tau) \mathbf{x}_{i t}^{*} \mathcal{G}_{i t}$. Following analogous arguments with the proof of Lemma A.5.(1), we can show that Lemma A.5.(2) holds.

(3) Recall that $R_{i t}=\mathbf{s}_{0}\left(\tau_{t}\right) \mathbf{y}_{i} \cdot-\sum_{l=1}^{p} \mathbf{x}_{i}^{(l)} \beta_{l}\left(\tau_{t}\right)$ and $\mathcal{R}_{i t}=R_{i t}-\sum_{j=1}^{J} \widehat{\mathcal{P}}_{j t}^{R}\left(V_{j i t}\right)$. Define

$$
\begin{aligned}
& \mathcal{H}_{N,(t, s)}^{\gamma}(\mathbf{v})=\widehat{E}\left[\gamma_{s}\left(\mathbf{V}_{i s}\right) \mid \mathbf{V}_{i t}=\mathbf{v}\right], \quad \mathcal{H}_{N,(t, s)}^{\varepsilon}(\mathbf{v})=\widehat{E}\left[\varepsilon_{i s} \mid \mathbf{V}_{i t}=\mathbf{v}\right], \\
& \widehat{\mathcal{P}}_{j(t, s)}^{\gamma}\left(v_{j}\right)=\frac{1}{N} \sum_{i=1}^{N} \mathcal{H}_{N,(t, s)}^{\gamma}\left(\mathbf{V}_{i t}^{(-j)}\left(v_{j}\right)\right) W^{(-j)}\left(\mathbf{V}_{i t}^{(-j)}\right) w_{j}\left(v_{j}\right), \\
& \widehat{\mathcal{P}}_{j(t, s)}^{\varepsilon}\left(v_{j}\right)=\frac{1}{N} \sum_{i=1}^{N} \mathcal{H}_{N,(t, s)}^{\varepsilon}\left(\mathbf{V}_{i t}^{(-j)}\left(v_{j}\right)\right) W^{(-j)}\left(\mathbf{V}_{i t}^{(-j)}\right) w_{j}\left(v_{j}\right) .
\end{aligned}
$$

By simple calculation, we have

$$
\sum_{i=1}^{N} \sum_{t=1}^{T} K_{t}(\tau) \mathbf{x}_{i t}^{*} \mathcal{R}_{i t}=\sum_{i=1}^{N} \sum_{t=1}^{T} K_{t}(\tau) \mathbf{x}_{i t}^{*} \mathcal{R}_{i t}^{(1)}+\sum_{i=1}^{N} \sum_{t=1}^{T} K_{t}(\tau) \mathbf{x}_{i t}^{*} \mathcal{R}_{i t}^{(2)}+O_{P}\left(N T h_{0} b^{2}\right),
$$

where $\mathcal{R}_{i t}^{(1)}=\frac{1}{T b} \sum_{s=1}^{T} K_{s}\left(\tau_{t}\right) \sum_{j=1}^{J}\left(\gamma_{j s}\left(V_{j i s}\right)-\mathcal{P}_{j(t, s)}^{\gamma}\left(V_{j i t}\right)\right)$ and $\mathcal{R}_{i t}^{(2)}=\frac{1}{T b} \sum_{s=1}^{T} K_{s}\left(\tau_{t}\right)\left(\varepsilon_{i s}-\mathcal{P}_{j(t, s)}^{\varepsilon}\left(V_{j i t}\right)\right)$.

Analogously to the proof of Lemmas A.5.(1) and A.5.(2), we can easily show that

$$
\sum_{i=1}^{N} \sum_{t=1}^{T} K_{t}(\tau) \mathbf{x}_{i t}^{*} \mathcal{R}_{i t}^{(1)}=O_{P}\left(N T h_{0}\left(\sum_{k=1}^{J} h_{k}^{2}\right)\right) .
$$

For $\mathcal{R}_{i t}^{(2)}$, since $\varepsilon_{i t}$ is independent with $\mathbf{x}_{i t}^{*}$, by the $\alpha$-mixing conditions in Assumption 2, we have

$$
\sum_{i=1}^{N} \sum_{t=1}^{T} K_{t}(\tau) \mathbf{x}_{i t}^{*} \mathcal{R}_{i t}^{(2)}=o_{P}\left(N T h_{0}\left(b^{2}+\sum_{k=1}^{J} h_{k}^{2}\right)\right) .
$$

Therefore, we have Lemma B.2.(3) holds.

\section{Proof of Lemma A.6}

Recall that $\mathcal{E}_{i t}=\varepsilon_{i t}-\sum_{j=1}^{J} \widehat{\mathcal{P}}_{j t}^{\varepsilon}\left(V_{j i t}\right)$ and $\widehat{\mathcal{P}}_{j t}^{\varepsilon}\left(v_{j}\right)=\frac{1}{N} \sum_{i=1}^{N} \mathcal{H}_{N, t}^{\varepsilon}\left(\mathbf{V}_{i t}^{(-j)}\left(v_{j}\right)\right) W^{(-j)}\left(\mathbf{V}_{i t}^{(-j)}\right) w_{j}\left(v_{j}\right)$. We consider the local linear estimator:

$$
\mathcal{H}_{N, t}^{\varepsilon}(\mathbf{v})=\mathbf{s}_{t}(\mathbf{v}) \varepsilon_{\cdot t},
$$

where $\mathbf{s}_{t}(\mathbf{v})=\left[1, \mathbf{0}_{1 \times J}\right]\left(\mathcal{M}_{t}^{\top}(\mathbf{v}) \mathcal{W}_{t}(\mathbf{v}) \mathcal{M}_{t}(\mathbf{v})\right)^{-1} \mathcal{M}_{t}^{\top}(\mathbf{v}) \mathcal{W}_{t}(\mathbf{v})$ and $\varepsilon_{\cdot t}=\left(\varepsilon_{1 t}, \varepsilon_{2 t}, \ldots, \varepsilon_{N t}\right)^{\top}$.

For each $j$, we have

$$
\widehat{\mathcal{P}}_{j t}^{\varepsilon}\left(V_{j i t}\right)=\frac{1}{N} \sum_{n=1}^{N} W^{(-j)}\left(\mathbf{V}_{n t}^{(-j)}\right) w_{j}\left(V_{j i t}\right) \mathbf{s}_{t}\left(\mathbf{V}_{n t}^{(-j)}\left(V_{j i t}\right)\right) \varepsilon_{\cdot t} .
$$

Recall that $x_{i t}^{(l) *}=\widetilde{x}_{i t}^{(l)}-\sum_{j=1}^{J} \mathcal{P}_{j t}^{(l)}\left(V_{j i t}\right), \breve{x}_{i t}^{(l)}=\widetilde{x}_{i t}^{(l)}-\mathcal{H}_{t}^{(l)}\left(\mathbf{V}_{i t}\right)$ and $\breve{x}_{i t}^{(l) c}=\mathcal{H}_{t}^{(l)}\left(\mathbf{V}_{i t}\right)-\sum_{j=1}^{J} \mathcal{P}_{j t}^{(l)}\left(V_{j i t}\right)$ 
Recall that $\widetilde{x}_{i t}^{(l)}=x_{i t}^{(l)}-H_{t}^{(l)}\left(\mathbf{V}_{i t}\right)$ and $\widetilde{x}_{i t}^{(l) c}=H_{t}^{(l)}\left(\mathbf{V}_{i t}\right)-\sum_{j=1}^{J} \mathcal{P}_{j t}^{(l)}\left(V_{j i t}\right)$. By Lemma B.1,

$$
\begin{aligned}
& \sum_{i=1}^{N} \breve{x}_{i t}^{(l) c} \widehat{\mathcal{P}}_{j t}^{\varepsilon}\left(V_{j i t}\right)=\frac{1}{N^{2} H_{J}} \sum_{n=1}^{N} \sum_{m=1}^{N} \sum_{i=1}^{N} \breve{x}_{i t}^{(l) c} W^{(-j)}\left(\mathbf{V}_{n t}^{(-j)}\right) w_{j}\left(V_{j i t}\right) p_{V}^{-1}\left(\mathbf{V}_{n t}^{(-j)}\left(V_{j i t}\right)\right) K\left(\frac{V_{j m t}-V_{j i t}}{h_{j}}\right) \\
& \cdot \prod_{k \neq j} K\left(\frac{V_{k m t}-V_{k n t}}{h_{k}}\right) \varepsilon_{m t}\left(1+o_{P}(1)\right) \\
& =\frac{1}{N h_{j}} \sum_{m=1}^{N} \sum_{i=1}^{N} \widetilde{x}_{i t}^{(l) c} W^{(-j)}\left(\mathbf{V}_{m t}^{(-j)}\right) w_{j}\left(V_{j i t}\right) p_{V}^{-1}\left(\mathbf{V}_{m t}^{(-j)}\left(V_{j i t}\right)\right) p_{V}^{(-j)}\left(\mathbf{V}_{m t}^{(-j)}\right) K\left(\frac{V_{j m t}-V_{j i t}}{h_{j}}\right) \varepsilon_{m t} \\
& \cdot\left(1+o_{P}(1)\right) \\
& =\sum_{m=1}^{N} W^{(-j)}\left(\mathbf{V}_{m t}^{(-j)}\right) w_{j}\left(V_{j m t}\right) p_{V}^{-1}\left(\mathbf{V}_{m t}\right) p_{V}^{(-j)}\left(\mathbf{V}_{m t}^{(-j)}\right) p_{V, j}\left(V_{j m t}\right) \breve{x}_{m t}^{(l) c} \varepsilon_{m t}\left(1+o_{P}(1)\right) .
\end{aligned}
$$

Since $\breve{x}_{i t}^{(l)}$ is mean zero and uncorrelated with $\mathbf{V}_{i t}$, analogously to (B.17), we have

$$
\begin{aligned}
& \sum_{i=1}^{N} \sum_{t=1}^{T} K_{t, 0}(\tau) \breve{x}_{i t}^{(l)} \widehat{\mathcal{P}}_{j t}^{\varepsilon}\left(V_{j i t}\right)=\frac{1}{N h_{j}} \sum_{i=1}^{N} \sum_{m=1}^{N} \sum_{t=1}^{T} K_{t, 0}(\tau) \breve{x}_{i t}^{(l)} W^{(-j)}\left(\mathbf{V}_{m t}^{(-j)}\right) w_{j}\left(V_{j i t}\right) \\
& \times p_{V}^{-1}\left(\mathbf{V}_{m t}^{(-j)}\left(V_{j i t}\right)\right) p_{V}^{(-j)}\left(\mathbf{V}_{m t}^{(-j)}\right) K\left(\frac{V_{j m t}-V_{j i t}}{h_{j}}\right) \varepsilon_{m t}\left(1+o_{P}(1)\right) .
\end{aligned}
$$

It is clear that

$E\left[\sum_{i=1}^{N} \sum_{m=1}^{N} \sum_{t=1}^{T} K_{t, 0}(\tau) \breve{x}_{i t}^{(l)} W^{(-j)}\left(\mathbf{V}_{m t}^{(-j)}\right) w_{j}\left(V_{j i t}\right) p_{V}^{-1}\left(\mathbf{V}_{m t}^{(-j)}\left(V_{j i t}\right)\right) p_{V}^{(-j)}\left(\mathbf{V}_{m t}^{(-j)}\right) K\left(\frac{V_{j m t}-V_{j i t}}{h_{j}}\right) \varepsilon_{m t}\right]=0$.

In addition, by Assumption 2 and analogously to (B.3) and (B.15),

$$
\begin{aligned}
& E\left[\left|\sum_{i=1}^{N} \sum_{m=1}^{N} \sum_{t=1}^{T} K_{t, 0}(\tau) \breve{x}_{i t}^{(l)} W^{(-j)}\left(\mathbf{V}_{m t}^{(-j)}\right) w_{j}\left(V_{j i t}\right) p_{V}^{-1}\left(\mathbf{V}_{m t}^{(-j)}\left(V_{j i t}\right)\right) p_{V}^{(-j)}\left(\mathbf{V}_{m t}^{(-j)}\right) K\left(\frac{V_{j m t}-V_{j i t}}{h_{j}}\right) \varepsilon_{m t}\right|^{2}\right] \\
& \leq C h_{j} \sum_{i=1}^{N} \sum_{n=1}^{N} \sum_{m=1}^{N} \sum_{a=1}^{N} \sum_{t=1}^{T} \sum_{s=1}^{T} K_{t, 0}(\tau) K_{s, 0}(\tau)\left|E\left[\breve{x}_{i t}^{(l)} \breve{x}_{n s}^{(l)}\right]\right|\left|E\left[\varepsilon_{m t} \varepsilon_{a s}\right]\right|=O\left(N^{2} T h_{0} h_{j}\right) .
\end{aligned}
$$

Therefore, we have

$$
\sum_{i=1}^{N} \sum_{t=1}^{T} K_{t, 0}(\tau) \widetilde{x}_{i t}^{(l)} \widehat{\mathcal{P}}_{j t}^{\varepsilon}\left(V_{j i t}\right)=O_{P}\left(\sqrt{T h_{0} h_{j}^{-1}}\right)=o_{P}\left(\sqrt{N T h_{0}}\right) .
$$

Recall that $\phi_{j i t}=W^{(-j)}\left(\mathbf{V}_{i t}^{(-j)}\right) w_{j}\left(V_{j i t}\right) p_{V}^{-1}\left(\mathbf{V}_{i t}\right) p_{V}^{(-j)}\left(\mathbf{V}_{i t}^{(-j)}\right) p_{V, j}\left(V_{j i t}\right)$. By Lemma A.2, (B.17) and (B.18), we have

$$
\begin{aligned}
& \sum_{i=1}^{N} \sum_{t=1}^{T} K_{t, 0}(\tau) \mathbf{x}_{i t}^{*} \mathcal{E}_{i t}=\sum_{i=1}^{N} \sum_{t=1}^{T} K_{t, 0}(\tau) \mathbf{x}_{i t}^{*} \varepsilon_{i t}-\sum_{i=1}^{N} \sum_{t=1}^{T} \sum_{j=1}^{J} K_{t, 0}(\tau)\left(\breve{x}_{i t}^{(l)}+\breve{x}_{i t}^{(l) c}\right) \widehat{\mathcal{P}}_{j t}^{\varepsilon}\left(V_{j i t}\right) \\
& =\sum_{i=1}^{N} \sum_{t=1}^{T} K_{t, 0}(\tau) \mathbf{x}_{i t}^{*} \varepsilon_{i t}-\sum_{i=1}^{N} \sum_{t=1}^{T} \sum_{j=1}^{J} K_{t, 0}(\tau) \phi_{j i t} \breve{x}_{i t}^{(l) c} \varepsilon_{i t}+o_{P}\left(\sqrt{N T h_{0}}\right) \\
& =\sum_{i=1}^{N} \sum_{t=1}^{T} K_{t, 0}(\tau) \widetilde{\mathbf{x}}_{i t}^{*} \varepsilon_{i t}+o_{P}\left(\sqrt{N T h_{0}}\right),
\end{aligned}
$$

where $\widetilde{\mathbf{x}}_{i t}^{*}=\breve{x}_{i t}^{(l)}+\breve{x}_{i t}^{(l) c}\left(1-\sum_{j=1}^{J} \phi_{j i t}\right)$.

\section{B.2 Secondary lemmas and their proofs}

For the proofs of Lemmas A.1-A.4, we have used the following secondary lemmas. 
Recall that $\mu_{m}=\int v^{m} K(v) d v, K_{j i t, m}\left(v_{j}\right)=K\left(\frac{V_{j i t}-v_{j}}{h_{j}}\right)\left(\frac{V_{j i t}-v_{j}}{h_{j}}\right)^{m}$, for $m=0,1,2 ; H_{J}=\prod_{j=1}^{J} h_{j}$; $H_{J}^{(-j)}=\prod_{k \neq j}^{J} h_{k}, \mathbf{V}_{i t}^{(-j)}=\left(V_{1 i t}, \ldots, V_{j-1, i t}, V_{j+1, i t}, \ldots, V_{J i t}\right) ; \mathbf{V}_{i t}^{(-j)}\left(v_{j}\right)=\left(V_{1 i t}, \ldots, V_{j-1, i t}, v_{j}, V_{j+1, i t}, \ldots, V_{J i t}\right) ;$ $\mathbf{v}^{(-j)}=\left(v_{1}, v_{2}, \ldots, v_{j-1}, v_{j+1}, \ldots, v_{J}\right) ; p_{V, j}\left(v_{j}\right)$ is the marginal probability density function of $V_{j i t} ; p_{V}(\mathbf{v})$ and $p_{V}^{(-j)}\left(\mathbf{v}^{(-j)}\right)$ are the joint probability density functions of $\mathbf{V}_{i t}$ and $\mathbf{V}_{i t}^{(-j)}$, respectively.

Lemma B.1. Let Assumptions 1-3 hold. For any deterministic function $G(\mathbf{v})=G\left(v_{1}, v_{2}, \ldots, v_{J}\right)$ that has continuous derivatives of up to second order on the support $v_{j}$ in $\left[\underline{v}_{j}, \bar{v}_{j}\right]$,

(1) $\left(N H_{J}\right)^{-1} \sum_{i=1}^{N} \prod_{j=1}^{J} K_{j i t, m}\left(v_{j}\right) G\left(\mathbf{V}_{i t}\right)=\mu_{m}^{J} G(\mathbf{v}) p_{V}(\mathbf{v})+O_{P}\left(\left(N H_{J}\right)^{-1 / 2}\right)$, for $J \geq 2$;

(2) $\left(N H_{J}^{(-j)}\right)^{-1} \sum_{i=1}^{N} \prod_{k \neq j} K_{k i t, m}\left(v_{k}\right) G\left(\mathbf{V}_{i t}^{(-j)}\left(v_{j}\right)\right)=\mu_{m}^{J-1} G(\mathbf{v}) p_{V}^{(-j)}\left(\mathbf{v}^{(-j)}\right)+O_{P}\left(\left(N H_{J}^{(-j)}\right)^{-1 / 2}\right)$, for $J \geq$ 2 ;

(3) $\left(N h_{j}\right)^{-1} \sum_{i=1}^{N} K_{j i t, m}\left(v_{j}\right) G\left(\mathbf{v}^{(-j)}\left(V_{j i t}\right)\right)=\mu_{m} G(\mathbf{v}) p_{V, j}\left(v_{j}\right)+o_{p}(1)$;

(4) $\left(N H_{J}\right)^{-1} \mathcal{M}_{t}^{\top}(\mathbf{v}) \mathcal{W}_{t}(\mathbf{v}) \mathcal{M}_{t}(\mathbf{v})-\boldsymbol{\Omega}^{A}(\mathbf{v})=o_{P}(1)$, where

$$
\boldsymbol{\Omega}^{A}(\mathbf{v})=\left(\begin{array}{cc}
p_{V}(\mathbf{v}) & \mathbf{0}_{1 \times J} \\
\mathbf{0}_{J \times 1} & \mu_{2} p_{V}(\mathbf{v}) \mathbf{I}_{J}
\end{array}\right) .
$$

Lemma B.2. Let Assumptions 1-3 hold. We have

(1) $\sum_{i=1}^{N} \sum_{n=1}^{N} \sum_{m=1}^{N} p_{V}^{-1}\left(\mathbf{V}_{n t}^{(-j)}\left(V_{j i t}\right)\right) K_{j m t, 0}\left(V_{j i t}\right) \prod_{k \neq j} K_{k m t, 0}\left(V_{k n t}\right)\left(\gamma_{j t}\left(V_{j m t}\right)-\gamma_{j t}\left(V_{j i t}\right)\right)=O_{P}\left(N^{3} H_{J} h_{j}^{2}\right)$.

(2) $\sum_{i=1}^{N} \sum_{n=1}^{N} \sum_{m=1}^{N} p_{V}^{-1}\left(\mathbf{V}_{n t}^{(-j)}\left(V_{j i t}\right)\right) K_{j m t, 0}\left(V_{j i t}\right) \prod_{k \neq j} K_{k m t, 0}\left(V_{k n t}\right) \gamma_{j t}\left(V_{b m t}\right)=O_{P}\left(N^{3} H_{J} h_{b}^{2}\right)$, for $b \neq j$.

\section{Proof of Lemma B.1}

(1)Let $\mathbf{x}=\left(x_{1}, x_{2}, \ldots, x_{J}\right)$ and $\mathbf{z}=\left(z_{1}, z_{2}, \ldots, z_{J}\right)$. It is clear to see that

$$
\begin{aligned}
& E\left[\frac{1}{N H_{J}} \sum_{i=1}^{N} \prod_{j=1}^{J} K_{j i t, m}\left(v_{j}\right) G\left(\mathbf{V}_{i t}\right)\right]=\frac{1}{H_{J}} E\left[\prod_{j=1}^{J} K\left(\frac{V_{j i t}-v_{j}}{h_{j}}\right)\left(\frac{V_{j i t}-v_{j}}{h_{j}}\right)^{m} G\left(V_{1 i t}, \ldots, V_{J i t}\right)\right] \\
& =\frac{1}{H_{J}} \int_{\mathbf{x}} \prod_{j=1}^{J} K\left(\frac{x_{j}-v_{j}}{h_{j}}\right)\left(\frac{x_{j}-v_{j}}{h_{j}}\right)^{m} G(\mathbf{x}) p_{V}(\mathbf{x}) d \mathbf{x} \\
& =\int_{\mathbf{z}} \prod_{j=1}^{J} K\left(z_{j}\right) z_{j}^{m} G\left(v_{1}+z_{1} h_{1}, \ldots, v_{J}+z_{J} h_{J}\right) p_{V}\left(v_{1}+z_{1} h_{1}, \ldots, v_{J}+z_{J} h_{J}\right) d \mathbf{z} .
\end{aligned}
$$

By Taylor expansion, we have

$$
G\left(v_{1}+z_{1} h_{1}, \ldots, v_{J}+z_{J} h_{J}\right)=G(\mathbf{v})+\sum_{j=1}^{J} \frac{\partial G}{\partial v_{j}}(\mathbf{v}) z_{j} h_{j}+\frac{1}{2} \sum_{j, k=1}^{J} \frac{\partial^{2} G}{\partial v_{j} \partial v_{k}}(\mathbf{v}) z_{j} z_{k} h_{j} h_{k}+o\left(\sum_{j=1}^{J} h_{j}^{2}\right),
$$

and

$$
p_{V}\left(v_{1}+z_{1} h_{1}, \ldots, v_{J}+z_{J} h_{J}\right)=p_{V}(\mathbf{v})+\sum_{j=1}^{J} \frac{\partial p_{V}}{\partial v_{j}}(\mathbf{v}) z_{j} h_{j}+\frac{1}{2} \sum_{j, k=1}^{J} \frac{\partial^{2} p_{V}}{\partial v_{j} \partial v_{k}}(\mathbf{v}) z_{j} z_{k} h_{j} h_{k}+o\left(\sum_{j=1}^{J} h_{j}^{2}\right) .
$$

Therefore, we have

$$
\begin{aligned}
& G\left(v_{1}+z_{1} h_{1}, \ldots, v_{J}+z_{J} h_{J}\right) p_{V}\left(v_{1}+z_{1} h_{1}, \ldots, v_{J}+z_{J} h_{J}\right)=G(\mathbf{v}) p_{V}(\mathbf{v})+p_{V}(\mathbf{v}) \sum_{j=1}^{J} \frac{\partial G}{\partial v_{j}}(\mathbf{v}) z_{j} h_{j} \\
& +G(\mathbf{v}) \sum_{j=1}^{J} \frac{\partial p_{V}}{\partial v_{j}}(\mathbf{v}) z_{j} h_{j}+O\left(\sum_{j=1}^{J} h_{j}^{2}\right) .
\end{aligned}
$$


It implies that

$$
\begin{aligned}
& E\left[\frac{1}{N H_{J}} \sum_{i=1}^{N} \prod_{j=1}^{J} K_{j i t, m}\left(v_{j}\right) G\left(\mathbf{V}_{i t}\right)\right]=\int_{\mathbf{z}} \prod_{j=1}^{J} K\left(z_{j}\right) z_{j}^{m} d \mathbf{z} G(\mathbf{v}) p_{V}(\mathbf{v}) \\
& +\sum_{j=1}^{J} \int_{\mathbf{z}} \prod_{k=1}^{J} K\left(z_{k}\right) z_{k}^{m} z_{j} d \mathbf{z} p_{V}(\mathbf{v}) \frac{\partial G}{\partial v_{j}}(\mathbf{v}) h_{j}+\sum_{j=1}^{J} \int_{\mathbf{z}} \prod_{k=1}^{J} K\left(z_{k}\right) z_{k}^{m} z_{j} d \mathbf{z} G(\mathbf{v}) \frac{\partial p_{V}}{\partial v_{j}}(\mathbf{v}) h_{j}+O\left(\sum_{j=1}^{J} h_{j}^{2}\right) \\
& =\mu_{m}^{J} G(\mathbf{v}) p_{V}(\mathbf{v})+\mu_{m}^{J-1} \mu_{m+1} \sum_{j=1}^{J} h_{j} \frac{\partial\left(G \cdot p_{V}\right)}{\partial v_{j}}(\mathbf{v})+O\left(\sum_{j=1}^{J} h_{j}^{2}\right)=\mu_{m}^{J} G(\mathbf{v}) p_{V}(\mathbf{v})+O\left(\sum_{j=1}^{J} h_{j}^{2}\right)
\end{aligned}
$$

where the last step holds for $J \geq 2$.

Let $\mathbf{x}^{(1)}=\left(x_{1}^{(1)}, x_{2}^{(1)}, \ldots, x_{J}^{(1)}\right) \mathbf{x}^{(2)}=\left(x_{1}^{(2)}, x_{2}^{(2)}, \ldots, x_{J}^{(2)}\right), \mathbf{z}^{(1)}=\left(z_{1}^{(1)}, z_{2}^{(1)}, \ldots, z_{J}^{(1)}\right)$ and $\mathbf{z}^{(2)}=\left(z_{1}^{(2)}, z_{2}^{(1)}, \ldots, z_{J}^{(2)}\right)$. Let $p_{V}^{(i, n)}\left(\mathbf{x}^{(1)}, \mathbf{x}^{(2)}\right)$ be the joint probability density function of $\mathbf{V}_{i t}$ and $\mathbf{V}_{n t}$. By the $\alpha$-mixing condition in Assumption 2 , we have

$$
\sum_{i=1}^{N} \sum_{n \neq i}\left|p_{V}^{(i, n)}(\mathbf{v}, \mathbf{v})-p_{V}^{2}(\mathbf{v})\right|=O(N) .
$$

For its second moment, we have

$$
\begin{aligned}
& E\left[\left|\frac{1}{N H_{J}} \sum_{i=1}^{N} \prod_{j=1}^{J} K_{j i t, m}\left(v_{j}\right) G\left(\mathbf{V}_{i t}\right)\right|^{2}\right]=\frac{1}{\left(N H_{J}\right)^{2}} \sum_{i=1}^{N} \sum_{n=1}^{N} E\left[\prod_{j=1}^{J} \prod_{k=1}^{J} K_{j i t, m}\left(v_{j}\right) K_{j n t, m}\left(v_{k}\right) G\left(\mathbf{V}_{i t}\right) G\left(\mathbf{V}_{n t}\right)\right] \\
& =\frac{1}{\left(N H_{J}\right)^{2}} \sum_{i=1}^{N} E\left[\prod_{j=1}^{J} \prod_{k=1}^{J} K_{j i t, m}\left(v_{j}\right) K_{j i t, m}\left(v_{k}\right) G\left(\mathbf{V}_{i t}\right)^{2}\right] \\
& +\frac{1}{\left(N H_{J}\right)^{2}} \sum_{i=1}^{N} \sum_{n \neq i} E\left[\prod_{j=1}^{J} \prod_{k=1}^{J} K_{j i t, m}\left(v_{j}\right) K_{j n t, m}\left(v_{k}\right) G\left(\mathbf{V}_{i t}\right) G\left(\mathbf{V}_{n t}\right)\right]=M_{N t, 1}+M_{N t, 2} .
\end{aligned}
$$

For $M_{N t, 1}$,

$$
\begin{aligned}
& M_{N t, 1}=\frac{1}{N H_{J}^{2}} \int_{\mathbf{x}} \prod_{j=1}^{J} \prod_{k=1}^{J} K\left(\frac{x_{j}-v_{j}}{h_{j}}\right)\left(\frac{x_{j}-v_{j}}{h_{j}}\right)^{m} K\left(\frac{x_{k}-v_{k}}{h_{k}}\right)\left(\frac{x_{k}-v_{k}}{h_{j}}\right)^{m} G^{2}(\mathbf{x}) p_{V}(\mathbf{x}) d \mathbf{x} \\
& =\frac{1}{N H_{J}} \int_{\mathbf{z}} \prod_{j=1}^{J} \prod_{k=1}^{J} K\left(z_{j}\right) K\left(z_{k}\right) z_{j}^{m} z_{k}^{m} G^{2}\left(v_{1}+z_{1} h_{1}, \ldots, v_{J}+z_{J} h_{J}\right) p_{V}\left(v_{1}+z_{1} h_{1}, \ldots, v_{J}+z_{J} h_{J}\right) d \mathbf{x} \\
& =\frac{1}{N H_{J}} \mu_{m}^{2 J} G^{2}(\mathbf{v}) p_{V}(\mathbf{v})(1+o(1))=O\left(\frac{1}{N H_{J}}\right) .
\end{aligned}
$$

For $M_{N t, 2}$,

$$
\begin{aligned}
& M_{N t, 2}=\frac{1}{\left(N H_{J}\right)^{2}} \sum_{i=1}^{N} \sum_{n \neq i} \int_{\mathbf{x}^{(1)}, \mathbf{x}^{(2)}} \prod_{j=1}^{J} \prod_{k=1}^{J} K\left(\frac{x_{j}^{(1)}-v_{j}}{h_{j}}\right)\left(\frac{x_{j}^{(1)}-v_{j}}{h_{j}}\right)^{m} K\left(\frac{x_{k}^{(2)}-v_{k}}{h_{k}}\right)\left(\frac{x_{k}^{(2)}-v_{k}}{h_{j}}\right)^{m} \\
& \times G\left(\mathbf{x}^{(1)}\right) G\left(\mathbf{x}^{(2)}\right) p_{V}^{(i, n)}\left(\mathbf{x}^{(1)}, \mathbf{x}^{(2)}\right) d \mathbf{x}^{(1)} d \mathbf{x}^{(2)} \\
& =\frac{1}{N^{2}} \sum_{i=1}^{N} \sum_{n \neq i} \int_{\mathbf{z}^{(1)}, \mathbf{z}^{(2)}} \prod_{j=1}^{J} \prod_{k=1}^{J} z_{j}^{(1) m} z_{k}^{(2) m} K\left(z_{j}^{(1)}\right) K\left(z_{k}^{(2)}\right) G\left(v_{1}+z_{1}^{(1)} h_{1}, \ldots, v_{J}+z_{J}^{(1)} h_{J}\right) \\
& \times G\left(v_{1}+z_{1}^{(2)} h_{1}, \ldots, v_{J}+z_{J}^{(2)} h_{J}\right) p_{V}^{(i, n)}\left(v_{1}+z_{1}^{(1)} h_{1}, \ldots, v_{J}+z_{J}^{(1)} h_{J}, v_{1}+z_{1}^{(2)} h_{1}, \ldots, v_{J}+z_{J}^{(2)} h_{J}\right) d \mathbf{z}^{(1)} d \mathbf{z}^{(2)} \\
& =\frac{\mu_{m}^{2 J}}{N^{2}} \sum_{i=1}^{N} \sum_{n \neq i} G^{2}(\mathbf{v}) p_{V}^{(i, n)}(\mathbf{v}, \mathbf{v})\left(1+O\left(\sum_{j=1}^{J} h_{j}^{2}\right)\right)
\end{aligned}
$$

where the last equality holds for $J \geq 2$. 
By (B.20), we have

$$
\begin{aligned}
& M_{N t, 2}=\left(\mu_{m}^{2 J} G^{2}(\mathbf{v}) p_{V}^{2}(\mathbf{v})+\frac{\mu_{m}^{2 J}}{N^{2}} \sum_{i=1}^{N} \sum_{n \neq i} G^{2}(\mathbf{v})\left(p_{V}^{(i, n)}(\mathbf{v}, \mathbf{v})-p_{V}^{2}(\mathbf{v})\right)\right)\left(1+O\left(\sum_{j=1}^{J} h_{j}^{2}\right)\right) \\
& +O\left(\frac{1}{N}\right)=\mu_{m}^{2 J} G^{2}(\mathbf{v}) p_{V}^{2}(\mathbf{v})+O\left(\frac{1}{N}\right)+O\left(\sum_{j=1}^{J} h_{j}^{2}\right) .
\end{aligned}
$$

Therefore, we have

$$
E\left[\left|\frac{1}{N H_{J}} \sum_{i=1}^{N} \prod_{j=1}^{J} K_{j i t, m}\left(v_{j}\right) G\left(\mathbf{V}_{i t}\right)\right|^{2}\right]-\mu_{m}^{2 J} G^{2}(\mathbf{v}) p_{V}^{2}(\mathbf{v})=O\left(\frac{1}{N H_{J}}\right) .
$$

Jointly with (B.19), it implies that Lemma B.1.(1) holds.

(2) Following analogous arguments with the proof of Lemma B.1.(1), we can show Lemma B.1.(2) holds.

(3) It is clear to see that

$$
\begin{aligned}
& E\left[\frac{1}{N h_{j}} \sum_{i=1}^{N} K_{j i t, m}\left(v_{j}\right) G\left(\mathbf{v}^{(-j)}\left(V_{j i t}\right)\right)\right]=\frac{1}{h_{j}} \int_{x} K\left(\frac{x-v_{j}}{h_{j}}\right)\left(\frac{x-v_{j}}{h_{j}}\right)^{m} G\left(\mathbf{v}^{(-j)}(x)\right) p_{V, j}(x) d x \\
& =\int_{z} z^{m} K(z) G\left(\mathbf{v}^{(-j)}\left(v_{j}+z h_{j}\right)\right) p_{V, j}\left(v_{j}+z h_{j}\right) d z=\mu_{m} G(\mathbf{v}) p_{V, j}\left(v_{j}\right)+o(1)
\end{aligned}
$$

For its second moment:

$$
\begin{aligned}
& E\left[\left|\frac{1}{N h_{j}} \sum_{i=1}^{N} K_{j i t, m}\left(v_{j}\right) G\left(\mathbf{v}^{(-j)}\left(V_{j i t}\right)\right)\right|^{2}\right]=\frac{1}{\left(N h_{j}\right)^{2}} \sum_{i=1}^{N} \sum_{n=1}^{N} E\left[K_{j i t, m}\left(v_{j}\right) K_{j n t, m}\left(v_{j}\right) G\left(\mathbf{v}^{(-j)}\left(V_{j i t}\right)\right) G\left(\mathbf{v}^{(-j)}\left(V_{j n t}\right)\right)\right] \\
& =\frac{1}{\left(N h_{j}\right)^{2}} \sum_{i=1}^{N} E\left[K_{j i t, m}^{2}\left(v_{j}\right) G^{2}\left(\mathbf{v}^{(-j)}\left(V_{j i t}\right)\right)\right]+\frac{1}{\left(N h_{j}\right)^{2}} \sum_{i=1}^{N} \sum_{n \neq i} E\left[K_{j i t, m}\left(v_{j}\right) K_{j n t, m}\left(v_{j}\right) G\left(\mathbf{v}^{(-j)}\left(V_{j i t}\right)\right) G\left(\mathbf{v}^{(-j)}\left(V_{j n t}\right)\right)\right] \\
& =\mu_{m}^{2} G^{2}(\mathbf{v}) p_{V, j}^{2}\left(v_{j}\right)+O\left(\frac{1}{N h_{j}}\right)
\end{aligned}
$$

where the last equality can be proved analogously to (B.23). Therefore, Lemma B.1.(3) holds.

(4) Lemma B.1.(4) is a direct extension of Lemma B.1.(1). In detail, recall we have the following notation:

$$
\begin{aligned}
& \mathcal{V}_{i t}(\mathbf{v})=\left(\frac{V_{1 i t}-v_{1}}{h_{1}}, \frac{V_{2 i t}-v_{2}}{h_{2}}, \ldots, \frac{V_{J i t}-v_{J}}{h_{J}}\right)^{\top}, \quad \mathcal{K}\left(\mathcal{V}_{i t}(\mathbf{v})\right)=\prod_{j=1}^{J} K\left(\frac{V_{j i t}-v_{j}}{h_{j}}\right), \\
& \mathcal{W}_{t}(\mathbf{v})=\operatorname{diag}\left(\mathcal{K}\left(\mathcal{V}_{1 t}(\mathbf{v})\right), \ldots, \mathcal{K}\left(\mathcal{V}_{N t}(\mathbf{v})\right)\right), \quad \mathcal{M}_{t}(\mathbf{v})=\left(\begin{array}{ccc}
1 & \ldots & 1 \\
\mathcal{V}_{1 t}(\mathbf{v}) & \ldots & \mathcal{V}_{N t}(\mathbf{v})
\end{array}\right)^{\top} .
\end{aligned}
$$

With this notation, it is clear to see that

$$
\frac{1}{N H_{J}} \mathcal{M}_{t}^{\top}(\mathbf{v}) \mathcal{W}_{t}(\mathbf{v}) \mathcal{M}_{t}(\mathbf{v})=\left(\begin{array}{cc}
\frac{1}{N H_{J}} \sum_{i=1}^{N} \mathcal{K}\left(\mathcal{V}_{i t}(\mathbf{v})\right) & \frac{1}{N H_{J}} \sum_{i=1}^{N} \mathcal{K}\left(\mathcal{V}_{i t}(\mathbf{v})\right) \mathcal{V}_{i t}^{\top}(\mathbf{v}) \\
\frac{1}{N H_{J}} \sum_{i=1}^{N} \mathcal{K}\left(\mathcal{V}_{i t}(\mathbf{v})\right) \mathcal{V}_{i t}(\mathbf{v}) & \frac{1}{N H_{J}} \sum_{i=1}^{N} \mathcal{K}\left(\mathcal{V}_{i t}(\mathbf{v})\right) \mathcal{V}_{i t}(\mathbf{v}) \mathcal{V}_{i t}^{\top}(\mathbf{v})
\end{array}\right)
$$

By Lemma B.1.(1) and let $m=0$ and $G(\mathbf{v})=1$, we have $\frac{1}{N H_{J}} \sum_{i=1}^{N} \mathcal{K}\left(\mathcal{V}_{i t}(\mathbf{v})\right)=p_{V}(\mathbf{v})+o_{P}(1)$.

Analogously, we also have $\frac{1}{N H_{J}} \sum_{i=1}^{N} \mathcal{K}\left(\mathcal{V}_{i t}(\mathbf{v})\right) \mathcal{V}_{i t}(\mathbf{v})=\mathbf{0}_{J \times 1}+o_{P}(1)$ and $\frac{1}{N H_{J}} \sum_{i=1}^{N} \mathcal{K}\left(\mathcal{V}_{i t}(\mathbf{v})\right) \mathcal{V}_{i t}(\mathbf{v}) \mathcal{V}_{i t}^{\top}(\mathbf{v})=$ $\mu_{2} p_{V}(\mathbf{v}) \mathbf{I}_{J}+o_{P}(1)$.

Therefore, Lemma B.1.(4) holds.

\section{Proof of Lemma B.2}


(1) By Taylor expansion, it is clear that

$$
\gamma_{j t}\left(V_{j m t}\right)-\gamma_{j t}\left(V_{j i t}\right)=\left(\frac{V_{j m t}-V_{j i t}}{h_{j}}\right) \gamma_{j t}^{\prime}\left(V_{j i t}\right) h_{j}+\left(\frac{V_{j m t}-V_{j i t}}{h_{j}}\right)^{2}\left(\frac{1}{2} \gamma_{j t}^{\prime \prime}\left(V_{j i t}\right) h_{j}^{2}+o_{P}\left(h_{j}^{2}\right)\right) .
$$

Let $\mathbf{x}^{(3)}=\left(x_{1}^{(3)}, x_{2}^{(3)}, \ldots, x_{J}^{(3)}\right)$ and $\mathbf{z}^{(3)}=\left(z_{1}^{(3)}, z_{2}^{(3)}, \ldots, z_{J}^{(3)}\right)$. Let $p_{V}^{(i, m, n)}\left(\mathbf{x}^{(1)}, \mathbf{x}^{(2)}, \mathbf{x}^{(3)}\right)$ be the joint probability density function of $\left\{\mathbf{V}_{i t}, \mathbf{V}_{m t}, \mathbf{V}_{n t}\right\}$. In addition, define $\mathbf{x}^{(3,-j)}\left(v_{j}\right)=\left(x_{1}^{(3)}, \ldots, x_{j-1}^{(3)}, v_{j}, x_{j+1}^{(3)}, \ldots, x_{J}^{(3)}\right)$ and $\mathbf{z}^{(3,-j)}\left(v_{j}\right)=\left(z_{1}^{(3)}, \ldots, z_{j-1}^{(3)}, v_{j}, z_{j+1}^{(3)}, \ldots, z_{J}^{(3)}\right)$.

For $m \neq i$, we have

$$
\begin{aligned}
& E\left[p_{V}^{-1}\left(\mathbf{V}_{n t}^{(-j)}\left(V_{j i t}\right)\right) K_{j m t, 1}\left(V_{j i t}\right) \prod_{k \neq j} K_{k m t, 0}\left(V_{k n t}\right) \gamma_{j t}^{\prime}\left(V_{j i t}\right) h_{j}\right] \\
& =h_{j} \int_{\mathbf{x}^{(1)}, \mathbf{x}^{(2)}, \mathbf{x}^{(3)}} p_{V}^{-1}\left(\mathbf{x}^{(3,-j)}\left(x_{j}^{(1)}\right)\right) K\left(\frac{x_{j}^{(2)}-x_{j}^{(1)}}{h_{j}}\right)\left(\frac{x_{j}^{(2)}-x_{j}^{(1)}}{h_{j}}\right) \prod_{k \neq j} K\left(\frac{x_{k}^{(2)}-x_{k}^{(3)}}{h_{k}}\right) \\
& \times \gamma_{j t}^{\prime}\left(x_{j}^{(1)}\right) p_{V}^{(i, m, n)}\left(\mathbf{x}^{(1)}, \mathbf{x}^{(2)}, \mathbf{x}^{(3)}\right) d \mathbf{x}^{(1)} d \mathbf{x}^{(2)} d \mathbf{x}^{(3)} \\
& =H_{J} h_{j} \int_{\mathbf{z}^{(1)}, \mathbf{z}^{(2)}, \mathbf{z}^{(3)}} p_{V}^{-1}\left(\mathbf{z}^{(3,-j)}\left(z_{j}^{(1)}\right)\right) z_{j}^{(2)} K\left(z_{j}^{(2)}\right) \prod_{k \neq j} K\left(z_{k}^{(2)}\right) \gamma_{j t}^{\prime}\left(z_{j}^{(1)}\right) \\
& \times p_{V}^{(i, m, n)}\left(\mathbf{z}^{(1)}, z_{1}^{(3)}+z_{1}^{(2)} h_{1}, \ldots, z_{j-1}^{(3)}+z_{j-1}^{(2)} h_{j-1}, z_{j}^{(1)}+z_{j}^{(2)} h_{j}, z_{j+1}^{(3)}+z_{j+1}^{(2)} h_{j+1} \ldots, z_{J}^{(3)}+z_{J}^{(2)} h_{J}, \mathbf{z}^{(3)}\right) \\
& \times d \mathbf{z}^{(1)} d \mathbf{z}^{(2)} d \mathbf{z}^{(3)}=O\left(H_{J} h_{j}^{2}\right) .
\end{aligned}
$$

It implies that

$$
E\left[\sum_{n=1}^{N} \sum_{i=1}^{N} \sum_{m=1}^{N} p_{V}^{-1}\left(\mathbf{V}_{n t}^{(-j)}\left(V_{j i t}\right)\right) K_{j m t, 1}\left(V_{j i t}\right) \prod_{k \neq j} K_{k m t, 0}\left(V_{k n t}\right) \gamma_{j t}^{\prime}\left(V_{j i t}\right) h_{j}\right]=O\left(N^{3} H_{J} h_{j}^{2}\right) .
$$

For the second moment, analogously,

$$
\begin{aligned}
& E\left[\left|\sum_{n=1}^{N} \sum_{i=1}^{N} \sum_{m=1}^{N} p_{V}^{-1}\left(\mathbf{v}^{(-j)}\left(V_{j i t}\right)\right) K_{j m t, 1}\left(V_{j i t}\right) \prod_{k \neq j} K_{k m t, 0}\left(v_{k}\right) \gamma_{j t}^{\prime}\left(V_{j i t}\right) h_{j}\right|^{2}\right] \\
& =h_{j}^{2} \sum_{n=1}^{N} \sum_{q=1}^{N} \sum_{i=1}^{N} \sum_{m=1}^{N} E\left[p_{V}^{-1}\left(\mathbf{V}_{n t}^{(-j)}\left(V_{j i t}\right)\right) p_{V}^{-1}\left(\mathbf{V}_{q t}^{(-j)}\left(V_{j i t}\right)\right) K_{j m t, 1}^{2}\left(V_{j i t}\right) \prod_{k \neq j}\left(K_{k m t, 0}\left(V_{k n t}\right) K_{k m t, 0}\left(V_{k q t}\right)\right) \gamma_{j t}^{\prime 2}\left(V_{j i t}\right)\right] \\
& +h_{j}^{2} \sum_{n=1}^{N} \sum_{q=1}^{N} \sum_{i=1}^{N} \sum_{m=1}^{N} \sum_{a \neq i}^{N} E\left[p_{V}^{-1}\left(\mathbf{V}_{n t}^{(-j)}\left(V_{j i t}\right)\right) p_{V}^{-1}\left(\mathbf{V}_{q t}^{(-j)}\left(V_{j a t}\right)\right) K_{j m t, 1}\left(V_{j i t}\right) K_{j m t, 1}\left(V_{j a t}\right)\right. \\
& \left.\times \prod_{k \neq j}\left(K_{k m t, 0}\left(V_{k n t}\right) K_{k m t, 0}\left(V_{k q t}\right)\right) \gamma_{j t}^{\prime}\left(V_{j i t}\right) \gamma_{j t}^{\prime}\left(V_{j a t}\right)\right] \\
& +h_{j}^{2} \sum_{n=1}^{N} \sum_{q=1}^{N} \sum_{i=1}^{N} \sum_{m=1}^{N} \sum_{b \neq m}^{N} E\left[p_{V}^{-1}\left(\mathbf{V}_{n t}^{(-j)}\left(V_{j i t}\right)\right) p_{V}^{-1}\left(\mathbf{V}_{q t}^{(-j)}\left(V_{j i t}\right)\right) K_{j m t, 1}\left(V_{j i t}\right) K_{j b t, 1}\left(V_{j i t}\right)\right. \\
& \left.\times \prod_{k \neq j}\left(K_{k m t, 0}\left(V_{k n t}\right) K_{k b t, 0}\left(V_{k q t}\right)\right) \gamma_{j t}^{\prime 2}\left(V_{j i t}\right)\right] \\
& +h_{j}^{2} \sum_{n=1}^{N} \sum_{q=1}^{N} \sum_{i=1}^{N} \sum_{m=1}^{N} \sum_{a \neq i}^{N} \sum_{b \neq m}^{N} E\left[p_{V}^{-1}\left(\mathbf{V}_{n t}^{(-j)}\left(V_{j i t}\right)\right) p_{V}^{-1}\left(\mathbf{V}_{q t}^{(-j)}\left(V_{j a t}\right)\right) K_{j m t, 1}\left(V_{j i t}\right) K_{j b t, 1}\left(V_{j a t}\right)\right. \\
& \left.\times \prod_{k \neq j}\left(K_{k m t, 0}\left(V_{k n t}\right) K_{k b t, 0}\left(V_{k q t}\right)\right) \gamma_{j t}^{\prime}\left(V_{j i t}\right) \gamma_{j t}^{\prime}\left(V_{j a t}\right)\right] \\
& =O\left(N^{4} H_{J} h_{j}^{2}\right)+O\left(N^{5} H_{J} h_{j}^{4}\right)+O\left(N^{5} H_{J}^{2} h_{j}^{4}\right)+O\left(N^{6} H_{J}^{2} h_{j}^{4}\right) .
\end{aligned}
$$

Therefore, we have

$$
\sum_{n=1}^{N} \sum_{i=1}^{N} \sum_{m=1}^{N} p_{V}^{-1}\left(\mathbf{V}_{n t}^{(-j)}\left(V_{j i t}\right)\right) K_{j m t, 1}\left(V_{j i t}\right) \prod_{k \neq j} K_{k m t, 0}\left(V_{k n t}\right) \gamma_{j t}^{\prime}\left(V_{j i t}\right) h_{j}=O_{P}\left(N^{3} H_{J} h_{j}^{2}\right)
$$

Analogously, we have

$$
\sum_{n=1}^{N} \sum_{i=1}^{N} \sum_{m=1}^{N} p_{V}^{-1}\left(\mathbf{V}_{n t}^{(-j)}\left(V_{j i t}\right)\right) K_{j m t, 1}\left(V_{j i t}\right) \prod_{k \neq j} K_{k m t, 0}\left(V_{k n t}\right) \gamma_{j t}^{\prime \prime}\left(V_{j i t}\right) h_{j}^{2}=O_{P}\left(N^{3} H_{J} h_{j}^{2}\right)
$$


(2) For $b \neq j$,

$$
\begin{aligned}
& E\left[\sum_{i=1}^{N} \sum_{n=1}^{N} \sum_{m=1}^{N} p_{V}^{-1}\left(\mathbf{V}_{n t}^{(-j)}\left(V_{j i t}\right)\right) K_{j m t, 0}\left(V_{j i t}\right) \prod_{k \neq j} K_{k m t, 0}\left(V_{k n t}\right) \gamma_{j t}\left(V_{b m t}\right)\right] \\
& =\sum_{i=1}^{N} \sum_{n=1}^{N} \sum_{m=1}^{N} \int_{\mathbf{x}^{(1)}, \mathbf{x}^{(2)}, \mathbf{x}^{(3)}} p_{V}^{-1}\left(\mathbf{x}^{(3,-j)}\left(x_{j}^{(1)}\right)\right) K\left(\frac{x_{j}^{(2)}-x_{j}^{(1)}}{h_{j}}\right) \prod_{k \neq j} K\left(\frac{x_{k}^{(2)}-x_{k}^{(3)}}{h_{k}}\right) \gamma_{b t}\left(x_{b}^{(2)}\right) \\
& \times p_{V}^{(i, m, n)}\left(\mathbf{x}^{(1)}, \mathbf{x}^{(2)}, \mathbf{x}^{(3)}\right) d \mathbf{x}^{(1)} d \mathbf{x}^{(2)} d \mathbf{x}^{(3)} \\
& =H_{J} \sum_{i=1}^{N} \sum_{n=1}^{N} \sum_{m=1}^{N} \int_{\mathbf{z}^{(1)}, \mathbf{z}^{(2)}, \mathbf{z}^{(3)}} p_{V}^{-1}\left(\mathbf{z}^{(3,-j)}\left(z_{j}^{(1)}\right)\right) K\left(z_{j}^{(2)}\right) \prod_{k \neq j} K\left(z_{k}^{(2)}\right) \gamma_{b t}\left(z_{b}^{(3)}+z_{b}^{(2)} h_{b}\right) \\
& \times p_{V}^{(i, m, n)}\left(\mathbf{z}^{(1)}, z_{1}^{(3)}+z_{1}^{(2)} h_{1}, \ldots, z_{j-1}^{(3)}+z_{j-1}^{(2)} h_{j-1}, z_{j}^{(1)}+z_{j}^{(2)} h_{j}, z_{j+1}^{(3)}+z_{j+1}^{(2)} h_{j+1} \ldots, z_{J}^{(3)}+z_{J}^{(2)} h_{J}, \mathbf{z}^{(3)}\right) \\
& \times d \mathbf{z}^{(1)} d \mathbf{z}^{(2)} d \mathbf{z}^{(3)}=O\left(N^{3} H_{J} h_{b}^{2}\right)
\end{aligned}
$$

where the last equality holds by Taylor expansion and the fact that

$$
\begin{aligned}
& H_{J} \sum_{i=1}^{N} \sum_{n=1}^{N} \sum_{m=1}^{N} \int_{\mathbf{z}^{(1)}, \mathbf{z}^{(2)}, \mathbf{z}^{(3)}} p_{V}^{-1}\left(\mathbf{z}^{(3,-j)}\left(z_{j}^{(1)}\right)\right) \gamma_{b t}\left(z^{(3)}\right) p_{V}^{(i, m, n)}\left(\mathbf{z}^{(1)}, \mathbf{z}^{(3,-j)}\left(z_{j}^{(1)}\right), \mathbf{z}^{(3)}\right) d \mathbf{z}^{(1)} d \mathbf{z}^{(2)} d \mathbf{z}^{(3)} \\
& =H_{J} \sum_{i=1}^{N} \sum_{n=1}^{N} \sum_{m=1}^{N} \int_{\mathbf{z}^{(1)}, \mathbf{z}^{(2)}, \mathbf{z}^{(3)}} p_{V}^{-1}\left(\mathbf{z}^{(3,-j)}\left(z_{j}^{(1)}\right)\right)\left(p_{V}^{(i, m, n)}\left(\mathbf{z}^{(1)}, \mathbf{z}^{(3,-j)}\left(z_{j}^{(1)}\right), \mathbf{z}^{(3)}\right)\right. \\
& \left.-p_{V}\left(\mathbf{z}^{(3,-j)}\left(z_{j}^{(1)}\right)\right) p_{V}^{(i, n)}\left(\mathbf{z}^{(1)}, \mathbf{z}^{(3)}\right)\right) \gamma_{b t}\left(z^{(3)}\right) d \mathbf{z}^{(1)} d \mathbf{z}^{(2)} d \mathbf{z}^{(3)}+N^{3} H_{J} E\left[\gamma_{b t}\left(V_{b n t}\right)\right]=O\left(N^{2} H_{J}\right) .
\end{aligned}
$$

Analogously, we can show that the second moment has the following order:

$$
E\left[\left|\sum_{i=1}^{N} \sum_{n=1}^{N} \sum_{m=1}^{N} p_{V}^{-1}\left(\mathbf{V}_{n t}^{(-j)}\left(V_{j i t}\right)\right) K_{j m t, 0}\left(V_{j i t}\right) \prod_{k \neq j} K_{k m t, 0}\left(V_{k n t}\right) \gamma_{j t}\left(V_{b m t}\right)\right|^{2}\right]=O\left(N^{6} H_{J}^{2} h_{b}^{4}\right) .
$$

Therefore, Lemma B.2.(2) holds.

\section{Appendix C Additional technicalities}

\section{C.1 Estimation of covariance matrices}

Let $\left\{\widehat{\varepsilon}_{i t}\right\}$ be the estimation residuals. With nonparametric estimates of density functions: $\widehat{p}_{V, j}\left(v_{j}\right), \widehat{p}_{V}^{(-j)}\left(\mathbf{v}^{(-j)}\right)$ and $p_{V}(\mathbf{v})$, we can construct estimates for $\xi_{j i t}\left(v_{j}\right)$ and $\phi_{j i t}$ :

$$
\widehat{\xi}_{j i t}\left(v_{j}\right)=\widehat{p}_{V}^{-1}\left(\mathbf{V}_{i t}^{(-j)}\left(v_{j}\right)\right) \widehat{p}_{V}^{(-j)}\left(\mathbf{V}_{i t}^{(-j)}\right) W^{(-j)}\left(\mathbf{V}_{i t}^{(-j)}\right) w_{j}\left(v_{j}\right), \quad \widehat{\phi}_{j i t}=\widehat{p}_{V, j}\left(V_{j i t}\right) \widehat{\xi}_{j i t} .
$$

With $\widehat{\phi}_{j i t}$, we can estimate $\widetilde{x}_{i t}^{(l) *}$ by $\widehat{\widetilde{x}}_{i t}^{(l) *}=\widehat{\widetilde{x}}_{i t}^{(l)}+\widehat{\widetilde{x}}_{i t}^{(l) c}\left(1-\sum_{j=1}^{J} \widehat{\phi}_{j i t}\right)$ where $\widehat{\widetilde{x}}_{i t}^{(l)}=x_{i t}^{(l)}-\mathcal{H}_{N, t}^{(l)}\left(\mathbf{V}_{i t}\right)$ and $\widehat{\widehat{x}}_{i t}^{(l) c}=\mathcal{H}_{N, t}^{(l)}\left(\mathbf{V}_{i t}\right)-\sum_{j=1}^{J} \widehat{\mathcal{P}}_{j t}^{(l)}\left(V_{j i t}\right)$.

We can construct estimates for $\boldsymbol{\Sigma}_{A}$ and $\boldsymbol{\Sigma}_{i n}(0)$ as follows:

$$
\widehat{\mathbf{\Sigma}}_{A}=\frac{1}{N T} \sum_{i=1}^{N} \sum_{t=1}^{T} \widehat{\mathbf{x}}_{i t}^{*} \widehat{\mathbf{x}}_{i t}^{* \top}, \quad \widehat{\boldsymbol{\Sigma}}_{i n}(0)=\frac{1}{T} \sum_{t=1}^{T} \widehat{\widetilde{\mathbf{x}}}_{i t}^{*} \widehat{\widetilde{\mathbf{x}}}_{n, t}^{* \top} .
$$

The estimates of $\boldsymbol{\Sigma}_{i n}(s)$ can be constructed analogously:

$$
\widehat{\boldsymbol{\Sigma}}_{i n}(s)=\frac{1}{T-s} \sum_{t=1}^{T-s} \widehat{\widetilde{\mathbf{x}}}_{i t}^{*} \widehat{\widetilde{\mathbf{x}}}_{n, t+s}^{* T} \text { for } s>0, \quad \widehat{\boldsymbol{\Sigma}}_{i n}(s)=\frac{1}{T+s} \sum_{t=1-s}^{T} \widehat{\widetilde{\mathbf{x}}}_{i t}^{*} \widehat{\widetilde{\mathbf{x}}}_{n, t+s}^{* \top} \text { for } s<0 .
$$


$\sigma_{i}^{2}$ and $\sigma_{i n}^{2}(s)$ can be estimated by

$$
\widehat{\sigma}_{i n}^{2}(s)=\frac{1}{T-s} \sum_{t=1}^{T-s} \widehat{\varepsilon}_{i 1} \widehat{\varepsilon}_{n, 1+s} \text { for } s \geq 0, \quad \widehat{\sigma}_{i n}^{2}(s)=\frac{1}{T+s} \sum_{t=1-s}^{T} \widehat{\varepsilon}_{i 1} \widehat{\varepsilon}_{n, 1+s} \text { for } s<0 .
$$

With $\widehat{\boldsymbol{\Sigma}}_{i n}(s)$ and $\widehat{\sigma}_{i n}^{2}(s)$, we can estimate $\boldsymbol{\Sigma}_{\beta}^{0}$ by:

$$
\widehat{\mathbf{\Sigma}}_{\beta}^{0}=\frac{1}{N} v_{0} \sum_{i=1}^{N} \sum_{n=1}^{N}\left(\sum_{s=1-T}^{T-1} \widehat{\mathbf{\Sigma}}_{i n}(s) \widehat{\sigma}_{i n}^{2}(s)\right),
$$

where $v_{0}$ can be calculated as $v_{0}=\int K(v)^{2} d v$ once the kernel function $K(v)$ is specified.

The estimation of $\Sigma_{j}^{\lambda}$ has more technical difficulty. For $\bar{F}_{j}$, we construct the sample estimate as:

$$
\widehat{\bar{F}}_{j}=\frac{1}{T} \sum_{t=1}^{T} \widehat{F}_{j t}
$$

With nonparametric probability density estimates $\widehat{p}_{V}(\mathbf{v})$ and $\widehat{p}_{V}^{(-j)}\left(\mathbf{v}^{(-j)}\right)$, we can estimate $\xi_{j i t}\left(v_{j}\right)$ by:

$$
\widehat{\xi}_{j i t}\left(v_{j}\right)=\widehat{p}_{V}^{-1}\left(\mathbf{V}_{i t}^{(-j)}\left(v_{j}\right)\right) \widehat{p}_{V}^{(-j)}\left(\mathbf{V}_{i t}^{(-j)}\right) W^{(-j)}\left(\mathbf{V}_{i t}^{(-j)}\right) w_{j}\left(v_{j}\right)
$$

We can provide a nonparametric estimate for $\widehat{\Sigma}_{j}^{V}\left(v_{j}\right)$ by the local constant or local linear method, where $\widehat{\boldsymbol{\Sigma}}_{i n}^{V}\left(v_{j}\right)$ is given by:

$$
\widehat{\boldsymbol{\Sigma}}_{i n}^{V}\left(v_{j}, u_{j}, s\right)=\widehat{E}\left[\widehat{\xi}_{j i 1}\left(v_{j}\right) \widehat{\xi}_{j n 1+s}\left(v_{j}\right) \mid V_{j i 1}=u_{j}, V_{j n, 1+s}=u_{j}\right] .
$$

With $\widehat{\boldsymbol{\Sigma}}_{i n}^{V}\left(v_{j}, u_{j}, s\right), \Sigma_{j}^{V}$ can be estimated by:

$$
\widehat{\Sigma}_{j}^{V}\left(v_{j}\right)=N^{-1} v_{0} \sum_{i=1}^{N} \sum_{n=1}^{N}\left(\sum_{s=1-T}^{T-1} \widehat{\boldsymbol{\Sigma}}_{i n}^{V}\left(v_{j}, v_{j}, s\right) \widehat{\sigma}_{i n}^{2}(s)\right) .
$$

\section{C.2 Nonparametric additive models with time-varying coefficients}

The proposed PMI method in Section 2 can also be used to estimate the following additive model when the factor structure is not included:

$$
y_{i t}=\mathbf{x}_{i t}^{\top} \boldsymbol{\beta}\left(\tau_{t}\right)+\sum_{j=1}^{J} \gamma_{j}\left(V_{j i t}\right)+\varepsilon_{i t} .
$$

Model (C.1) can be regarded as a special case of Model (2.3) when the factors are assumed to be constant $\left(F_{t j}=1\right)$. In this case, $\gamma_{j}\left(v_{j}\right)$ can be fully identified.

For estimate Model (C.1), we can follow Steps 1-2 of the estimation procedure in Section (2) and then obtain the estimator $\widehat{\gamma}_{j}\left(v_{j}\right)$ as follows:

$$
\begin{aligned}
\widehat{\gamma}_{j t}\left(v_{j}\right) & =\widehat{\mathcal{P}}_{j t}\left(v_{j}, \widehat{\boldsymbol{\beta}}\left(\tau_{t}\right)\right)-\frac{1}{N} \sum_{i=1}^{N} \widehat{\mathcal{P}}_{j t}\left(V_{j i t}, \widehat{\boldsymbol{\beta}}\left(\tau_{t}\right)\right), \\
\widehat{\gamma}_{j}\left(v_{j}\right) & =\frac{1}{T} \sum_{t=1}^{T} \widehat{\gamma}_{j t}\left(v_{j}\right) .
\end{aligned}
$$

For the estimator $\widehat{\gamma}_{j}\left(v_{j}\right)$, we can establish its asymptotic properties in the following corollary.

Corollary C.1 (CLT for $\left.\widehat{\gamma}_{j}\left(v_{j}\right)\right)$.

Let Assumptions 2-4 hold. As $N, T \rightarrow \infty$ simultaneously, if additionally $h_{0}^{2}+\sum_{k \neq j} h_{k}^{2}=o\left(h_{j}^{2}\right)$, then for each $j$ and $v_{j} \in\left(\underline{v}_{j}, \bar{v}_{j}\right)$ :

$$
\sqrt{N T h_{j}}\left(\widehat{\gamma}_{j}\left(v_{j}\right)-\gamma_{j}\left(v_{j}\right)-e_{j}\left(v_{j}\right) h_{j}^{2}\right) \stackrel{D}{\longrightarrow} \mathcal{N}\left(0, \Sigma_{j}^{V}\left(v_{j}\right)\right),
$$

where $e_{j}\left(v_{j}\right)=\frac{\mu_{2}}{2}\left(\gamma_{j}^{\prime \prime}\left(v_{j}\right)-E\left[\gamma_{j}^{\prime \prime}\left(V_{j i t}\right)\right]\right)\left(1+o_{P}(1)\right)$ and $\Sigma_{j}^{V}\left(v_{j}\right)$ is defined in Assumption 4 . 


\section{C.3 Stochastic factor models}

In Section 2, the factors are assumed to be unknown but deterministic for estimation purposes. In this section, we discuss about how to deal with the case where the factors are stochastic. We reconsider the estimation of Model (1.1) when the factors are stochastically stationary.

(1) Step 1: Recall $\widetilde{y}_{i t}=y_{i t}-m_{Y, i}\left(\tau_{t}\right), \widetilde{\mathbf{x}}_{i t}=\mathbf{x}_{i t}-\mathbf{m}_{X, i}\left(\tau_{t}\right)$, and the definition of $\gamma_{t}(\mathbf{v})$ for each given $t$ :

$$
\begin{aligned}
& \gamma_{t}\left(\mathbf{v}, \boldsymbol{\beta}_{t}\right)=E_{f}\left[y_{i t}-\boldsymbol{\beta}^{\top}\left(\tau_{t}\right) \mathbf{x}_{i t} \mid \mathbf{V}_{i t}=\mathbf{v}\right]=E_{f}\left[y_{i t}-m_{Y, i}\left(\tau_{t}\right)-\boldsymbol{\beta}^{\top}\left(\tau_{t}\right)\left(\mathbf{x}_{i t}-\mathbf{m}_{X, i}\left(\tau_{t}\right)\right) \mid \mathbf{V}_{i t}=\mathbf{v}\right] \\
& =E_{f}\left[\widetilde{y}_{i t} \mid \mathbf{V}_{i t}=\mathbf{v}\right]-\boldsymbol{\beta}^{\top}\left(\tau_{t}\right) E_{f}\left[\widetilde{\mathbf{x}}_{i t} \mid \mathbf{V}_{i t}=\mathbf{v}\right]=: \mathcal{H}_{t}^{(0)}(\mathbf{v})-\sum_{l=1}^{p} \beta_{l}\left(\tau_{t}\right) \mathcal{H}_{t}^{(l)}(\mathbf{v})
\end{aligned}
$$

where $\mathrm{E}_{f}(\cdot)$ is the conditional mean given $\mathbf{F}_{t}$.

The local linear estimator of $\gamma_{t}(\mathbf{v})$, given $\boldsymbol{\beta}_{t}$, can then be obtained by:

$$
\widehat{\gamma}_{t}(\mathbf{v}) \equiv \widehat{\gamma}_{t}\left(\mathbf{v}, \boldsymbol{\beta}_{t}\right)=\mathcal{H}_{N, t}^{(0)}(\mathbf{v})-\sum_{l=1}^{p} \beta_{l}\left(\tau_{t}\right) \mathcal{H}_{N, t}^{(l)}(\mathbf{v})
$$

where $\mathcal{H}_{N, t}^{(0)}(\mathbf{v})=\widehat{E}_{f}\left[\widehat{\widetilde{y}}_{i t} \mid \mathbf{V}_{i t}=\mathbf{v}\right]$ and $\mathcal{H}_{N, t}^{(l)}(\mathbf{v})=\widehat{E}_{f}\left[\widehat{\widetilde{x}}_{i t}^{(l)} \mid \mathbf{V}_{i t}=\mathbf{v}\right]$.

(2) Step 2: With the same $\mathbf{V}_{i t}^{(-j)}, \mathbf{V}_{i t}^{(-j)}\left(v_{j}\right), W^{(-j)}(\cdot)$ and $w_{j}(\cdot)$ being involved in the same way as in Step 2 of Section 2, we define the following MI projection function for $v_{j} \in\left[\bar{v}_{j}, \underline{v}_{j}\right]$,

$$
\begin{aligned}
& \mathcal{P}_{j t}\left(v_{j}, \boldsymbol{\beta}_{t}\right):=E_{f}\left[\gamma_{t}\left(\mathbf{V}_{i t}^{(-j)}\left(v_{j}\right), \boldsymbol{\beta}_{t}\right) W^{(-j)}\left(\mathbf{V}_{i t}^{(-j)}\right)\right] w_{j}\left(v_{j}\right) \\
& =\gamma_{j t}\left(v_{j}, \boldsymbol{\beta}_{t}\right) w_{j}\left(v_{j}\right)+\sum_{k \neq j} E_{f}\left[\gamma_{k t}\left(V_{k i t}, \boldsymbol{\beta}_{t}\right) W^{(-j)}\left(\mathbf{V}_{i t}^{(-j)}\right)\right]=\gamma_{j t}\left(v_{j}, \boldsymbol{\beta}_{t}\right)+C_{j},
\end{aligned}
$$

where $C_{j}=\sum_{k \neq j} E_{f}\left[\gamma_{k t}\left(V_{k i t}, \boldsymbol{\beta}_{t}\right) W^{(-j)}\left(\mathbf{V}_{i t}^{(-j)}\right)\right]$.

We still have $\gamma_{j t}\left(v_{j}, \boldsymbol{\beta}_{t}\right)=\mathcal{P}_{j t}\left(v_{j}, \boldsymbol{\beta}_{t}\right)-C_{j}$. For the MI projection function $\mathcal{P}_{j t}\left(v_{j}, \boldsymbol{\beta}_{t}\right)$, we define the following estimator:

$$
\widehat{\mathcal{P}}_{j t}\left(v_{j}, \boldsymbol{\beta}_{t}\right)=\frac{1}{N} \sum_{i=1}^{N} \widehat{\gamma}_{t}\left(\mathbf{V}_{i t}^{(-j)}\left(v_{j}\right), \boldsymbol{\beta}_{t}\right) W^{(-j)}\left(\mathbf{V}_{i t}^{(-j)}\right) w_{j}\left(v_{j}\right)=\widehat{\mathcal{P}}_{j t}^{(0)}\left(v_{j}\right)-\sum_{l=1}^{p} \beta_{l}\left(\tau_{t}\right) \widehat{\mathcal{P}}_{j t}^{(l)}\left(v_{j}\right),
$$

where $\widehat{\mathcal{P}}_{j t}^{(l)}\left(v_{j}\right)$ for $j=0,1, \cdots, p$ are the same as in Section 2.

(3) Step 3: This is the same as Step 3 in Section 2.

(4) Step 4: This is the same as Step 4 in Section 2.

The rest of the estimation procedure will be similar to those established in Section 2. It may then be shown in Section 3 that Theorems 3.1-3.3 hold conditionally given $\mathbf{F}$ and then they all hold with probability one by the bounded dominanted convergence theorem. 